\title{
MCNP5 Criticality Validation and Bias for Intermediate Enriched Uranium Systems
}

Prepared for the U.S. Department of Energy

Assistant Secretary for Environmental Management

Contractor for the U.S. Department of Energy under Contract DE-AC06-08RL14788

CH2MHILL

Plateau Remediation Company

P.O. Box 1600

Richland, Washington 99352 


\section{MCNP5 Criticality Validation and Bias for Intermediate Enriched Uranium Systems}

Document Type: TR

S. H. Finfrock

CH2M HILL Plateau Remediation Company

Date Published

December 2009

Prepared for the U.S. Department of Energy Assistant Secretary for Environmental Management

Contractor for the U.S. Department of Energy under Contract DE-AC06-08RL14788

\section{CH2MHILL}

Plateau Remediation Company

P.O. Box 1600

Richland, Washington
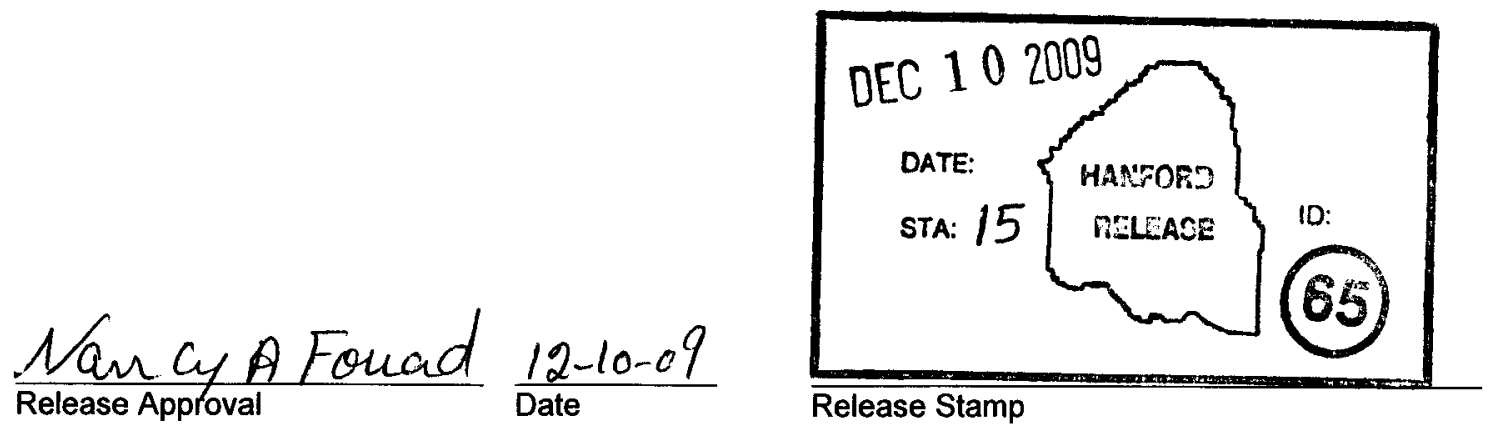

\section{Approved for Public Release; \\ Further Dissemination Unlimited}




\section{CHPRC-00455}

Revision 0

TRADEMARK DISCLAIMER

Reference herein to any specific commercial product, process,

or service by trade name, trademark, manufacturer, or

otherwise, does not necessarily constitute or imply its

endorsement, recommendation, or favoring by the United

States Government or any agency thereof or its contractors or subcontractors.

This report has been reproduced from the best available copy.

Printed in the United States of America

Total Pages:

75 
CHPRC-00455, REV. 0

\title{
MCNP5 \\ Criticality Validation and Bias for \\ Intermediate Enriched Uranium Systems
}

\author{
Prepared by \\ CH2M HILL Plateau Remediation Company \\ Richland, Washington
}

December 2009 
CHPRC-00455, REV. 0

This page is intentionally left blank. 


\section{MCNP5 Criticality Validation and Bias for Intermediate Enriched Uranium Systems}

May 2007

Prepared by

Fluor Government Group

Richland, Washington

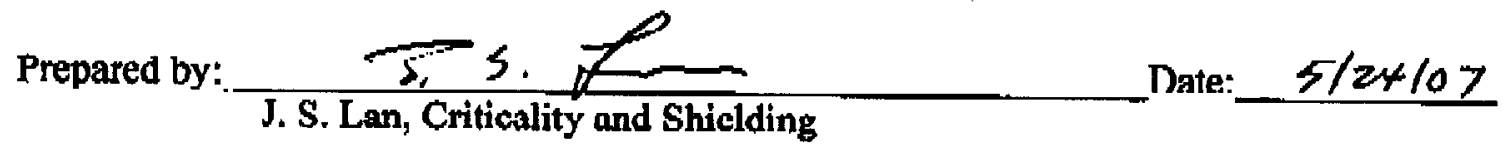

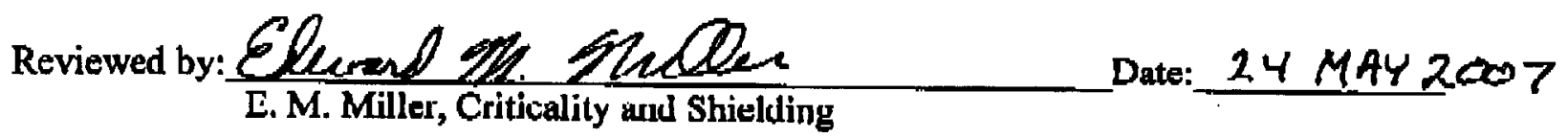

Approved by: Date:3/27/2008 
CHPRC-00455, REV. 0

This page is intentionally left blank. 


\section{TABLE OF CONTENTS}

1.0 INTRODUCTION

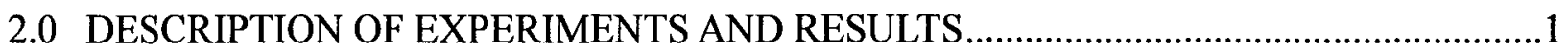

2.1 UPPER SUBCRITICAL LIMIT AND BENCHMARK SELECTION ..........................1

2.2 DESCRIPTION OF BENCHMARK CASES...............................................................

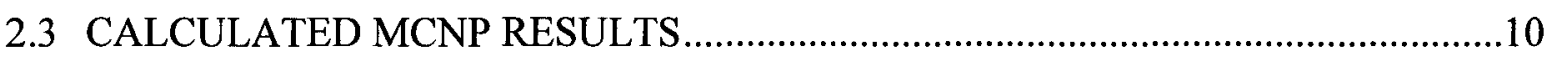

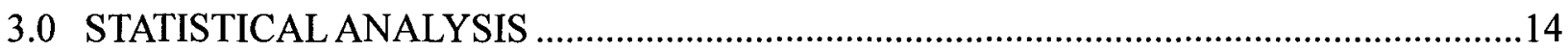

4.0 DISCUSSION OF CODE AND CROSS SECTIONS USED ………................................

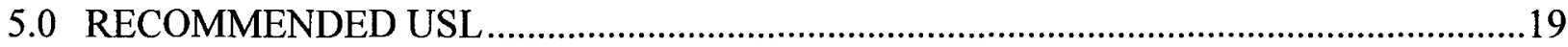

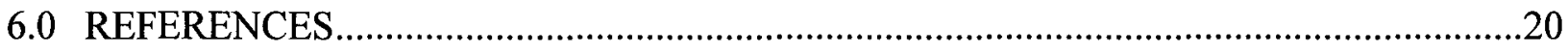

APPENDIX A - TECHNICAL PEER REVIEW …………..................................................

APPENDIX B - TABLE OF CROSS-SECTIONS UTILIZED …………………......................

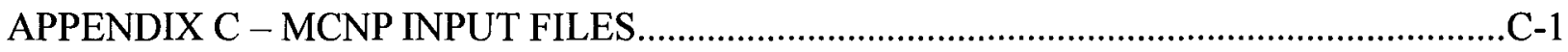

APPENDIX D - USLSTATS OUTPUTS ............................................................................ D-1

APPENDIX E - MANAGEMENT DECISION .................................................................. E-1

\section{LIST OF TABLES}

Table 2-1. Case Name and Short Description. 3

Table 2-2. Case Name and Reported Information ....................................................................

Table 2-3. Case Name and MCNP Results.........................................................................

Table B-1. MCNP Cross Section ID's Used in This Analysis ...................................................

\section{LIST OF FIGURES}

Figure 3-1. $\mathrm{k}_{\mathrm{eff}}$ Data and the USLSTATS Trending Results 


\section{LIST OF ACRONYMS}

$\begin{array}{ll}\text { AEG } & \text { Average Energy Group } \\ \text { CSER } & \text { Criticality Safety Evaluation Report } \\ \text { DVD } & \text { Digital Versatile Disc } \\ \text { EALF } & \text { Energy of Average Lethargy causing Fission } \\ \text { H/U } & \text { Hydrogen to Uranium ratio } \\ \text { H/X } & \text { Hydrogen to Fissile ratio } \\ \text { MCNP } & \text { Monte Carlo N-Particle } \\ \text { NEA } & \text { Nuclear Energy Agency } \\ \text { IEU } & \text { Intermediate Enriched Uranium } \\ \text { IEUCT } & \text { Intermediate Enriched Uranium, Compound, Thermal spectrum Systems } \\ \text { LANL } & \text { Los Alamos National Laboratory } \\ \text { LEUCT } & \text { Low Enriched Uranium, Compound, Thermal spectrum Systems } \\ \text { LEUST } & \text { Low Enriched Uranium, Solution, Thermal spectrum Systems } \\ \text { ORNL } & \text { Oak Ridge National Laboratory } \\ \text { PC } & \text { Personal Computer } \\ \text { PDF } & \text { electronic format } \\ \text { USL } & \text { Upper Subcritical Limit } \\ V_{m} / V_{f} & \text { Volume of Fuel Ratio }\end{array}$




\subsection{INTRODUCTION}

The purpose of this analysis is to validate the Monte Carlo N-Particle 5 (MCNP5) code Version 1.40 (LA-UR-03-1987, 2005) and its cross-section database for k-code calculations of intermediate enriched uranium systems on INTEL ${ }^{\circledR}$ processor based PC's running any version of the WINDOWS operating system. Configurations with intermediate enriched uranium were modeled with the moderator range of $39 \leq \mathrm{H} /$ Fissile $\leq 1438$. See Table $2-1$ for brief descriptions of selected cases and Table 3-1 for the range of applicability for this validation. A total of 167 input cases were evaluated including bare and reflected systems in a single body or arrays. The 167 cases were taken directly from the previous (Version 4C [Lan 2005]) validation database. Section 2.0 list data used to calculate $\mathrm{k}$-effective $\left(\mathrm{k}_{\mathrm{eff}}\right)$ for the 167 experimental criticality benchmark cases using the MCNP5 code v1.40 and its cross section database. Appendix B lists the MCNP cross-section database entries validated for use in evaluating the intermediate enriched uranium systems for criticality safety.

The dimensions and atom densities for the intermediate enriched uranium experiments were taken from NEA/NSC/DOC(95)03, September 2005, which will be referred to as the benchmark handbook throughout the report. For these input values, the experimental benchmark $\mathrm{k}_{\mathrm{eff}}$ is approximately 1.0. The MCNP validation computer runs ran to an accuracy of approximately \pm 0.001 . For the cases where the reported benchmark $k_{\text {eff }}$ was not equal to 1.0000 the MCNP calculational results were normalized. The difference between the MCNP validation computer runs and the experimentally measured $\mathrm{k}_{\text {eff }}$ is the MCNP5 v1.40 bias. The USLSTATS code (ORNL 1998) was utilized to perform the statistical analysis and generate an acceptable maximum $\mathrm{k}_{\text {eff }}$ limit for calculations of the intermediate enriched uranium type systems.

\subsection{DESCRIPTION OF EXPERIMENTS AND RESULTS}

\subsection{UPPER SUBCRITICAL LIMIT AND BENCHMARK SELECTION}

A common approach to ensuring subcriticality for a fissile unit is to determine a maximum $\mathrm{k}_{\text {eff }}$ limit below which all the k-code calculations of normal and abnormal conditions must fall. This limit is called the Upper Subcritical Limit (USL). This report performs validation studies, using critical experiments similar to actual or anticipated facility applications to determine the bias and uncertainty in the bias. The bias is a measure of the systematic differences between the MCNP calculational results and the experimental data. The uncertainty in the bias is a measure of both the accuracy and precision of the calculational and uncertainty in the experimental data.

Historically, certain parameters have been used to trend the bias because these are the parameters that have been found to have the greatest effect on the bias. These parameters include the moderator-to-fuel ratio (e.g., $\mathrm{H} / \mathrm{X}, \mathrm{V}_{\mathrm{m}} / \mathrm{V}_{\mathrm{f}}$ ), $\mathrm{Pu}$ to $\mathrm{Pu}+\mathrm{U}$ ratio (not applicable in this validation), and parameters that characterize the neutron energy spectrum (e.g., energy of

(B) INTEL is a registered trademark of the Intel Corporation, Santa Clara, California. 
average lethargy causing fission [EALF], average energy group [AEG]). Other parameters, such as material density or overall geometric shape, are generally considered to be of less importance in regard to a USL determination.

This report used benchmark cases selected from the handbook for validation analyses of the MCNP5 Version 1.40. Since the purpose of this evaluation is to support criticality safety analysis of intermediate enriched uranium fuels, cases were selected based on uranium being the sole fissile isotope. Benchmark experiments selected include cases being significantly moderated, as well as cases with little moderation to cover a broad neutron energy spectrum. Table 2-1 contains the case names and information on each benchmark case. Table 2-2 contains MCNP $\mathrm{k}_{\mathrm{eff}}$ results of this validation study.

\subsection{DESCRIPTION OF BENCHMARK CASES}

The benchmark handbook is a collection of evaluated experimental data representative of configurations encountered in the nuclear fuel cycle. The data is useful for the validation of neutronic codes and the associated nuclear data libraries used for criticality safety analysis.

The handbook is produced in electronic format (PDF files) where the experiments are grouped into evaluations, which are categorized by fissile media ( $\mathrm{Pu}$, Low Enriched Uranium, ...), fuel form (Solution, Metal, ...) and by neutron spectrum description (Thermal, Fast, ...). The evaluations are structured into different sections where the experimental program is described, the data are evaluated, the effect of experimental data uncertainties is assessed, and the benchmark models are discussed. Sample calculations are also presented and calculated spectral characteristics of the experiments are provided.

Table 2-1 below contains a list of the cases run (sorted by geometry identifier and case name) and a short description of each case. The moderator, reflector, $\mathrm{H} /{ }^{235} \mathrm{U}$ ratio, and form of uranium are included to demonstrate the range of benchmark experiments included in this validation. For the LEU-COMP-THERM-049 series of cases the H/U $\left({ }^{235} U+{ }^{238} \mathrm{U}\right)$ is given.

The given case name is directly related to the benchmark evaluation name from the handbook. IEUCT stands for Intermediate Enriched Uranium systems, Compound, Thermal neutron spectrum systems, LEUCT stands for Low Enriched Uranium systems, Compound, Thermal neutron spectrum systems and LEUST stands for Low Enriched Uranium systems, Solution, Thermal neutron spectrum systems, etc. But EU has been neglected in case names to shorten the column width in the following tables. The numbers stand for the specific evaluation number, and the experiment (or case) number taken from the NEA benchmark handbook. 
Table 2-1. Case Name and Short Description

\begin{tabular}{|c|c|c|c|c|c|}
\hline Case Name & Geometry & Form & Moderator & Reflector & $\mathbf{H} / \mathbf{U}$ \\
\hline ICT-002-001 & $\mathrm{UO}_{2}$ HEX Array with Annular Fuel Rods & $\mathrm{U}(17 \%) \mathrm{O}_{2}$ & $\mathrm{H}_{2} \mathrm{O}$ & $\mathrm{H}_{2} \mathrm{O}$ & $\mathrm{NA}$ \\
\hline ICT-002-002 & $\mathrm{UO}_{2}$ HEX Array with Annular Fuel Rods & $\mathrm{U}(17 \%) \mathrm{O}_{2}$ & $\mathrm{H}_{2} \mathrm{O}$ & $\mathrm{H}_{2} \mathrm{O}$ & NA \\
\hline ICT-002-003 & $\mathrm{UO}_{2} \mathrm{HEX}$ Array with Annular Fuel Rods & $\mathrm{U}(17 \%) \mathrm{O}_{2}$ & $\mathrm{H}_{2} \mathrm{O}$ & $\mathrm{H}_{2} \mathrm{O}$ & NA \\
\hline ICT-002-004 & $\mathrm{UO}_{2} \mathrm{HEX}$ Array with Annular Fuel Rods & $\mathrm{U}(17 \%) \mathrm{O}_{2}$ & $\mathrm{H}_{2} \mathrm{O}$ & $\mathrm{H}_{2} \mathrm{O}$ & NA \\
\hline ICT-002-005 & $\mathrm{UO}_{2}$ HEX Array with Annular Fuel Rods & $\mathrm{U}(17 \%) \mathrm{O}_{2}$ & $\mathrm{H}_{2} \mathrm{O}$ & $\mathrm{H}_{2} \mathrm{O}$ & $\mathrm{NA}$ \\
\hline ICT-002-006 & $\mathrm{UO}_{2}$ HEX Array with Annular Fuel Rods & $\mathrm{U}(17 \%) \mathrm{O}_{2}$ & $\mathrm{H}_{2} \mathrm{O}$ & $\mathrm{H}_{2} \mathrm{O}$ & NA \\
\hline LCT-002-001 & $\mathrm{UO}_{2}$ Fuel Rods with water reflector & $\mathrm{U}(4.31 \%) \mathrm{O}_{2}$ & $\mathrm{H}_{2} \mathrm{O}$ & $\mathrm{H}_{2} \mathrm{O}$ & NA \\
\hline LCT-002-002 & $\mathrm{UO}_{2}$ Fuel Rods with water reflector & $\mathrm{U}(4.31 \%) \mathrm{O}_{2}$ & $\mathrm{H}_{2} \mathrm{O}$ & $\mathrm{H}_{2} \mathrm{O}$ & $\mathrm{NA}$ \\
\hline LCT-002-003 & $\mathrm{UO}_{2}$ Fuel Rods with water reflector & $\mathrm{U}(4.31 \%) \mathrm{O}_{2}$ & $\mathrm{H}_{2} \mathrm{O}$ & $\mathrm{H}_{2} \mathrm{O}$ & $\mathrm{NA}$ \\
\hline LCT-002-004 & $\mathrm{UO}_{2}$ Fuel Rods with water reflector & $\mathrm{U}(4.31 \%) \mathrm{O}_{2}$ & $\mathrm{H}_{2} \mathrm{O}$ & $\mathrm{H}_{2} \mathrm{O}$ & $\mathrm{NA}$ \\
\hline LCT-002-005 & $\mathrm{UO}_{2}$ Fuel Rods with water reflector & $\mathrm{U}(4.31 \%) \mathrm{O}_{2}$ & $\mathrm{H}_{2} \mathrm{O}$ & $\mathrm{H}_{2} \mathrm{O}$ & $\mathrm{NA}$ \\
\hline LCT-004-001 & $\mathrm{UO}_{2}$ Fuel Rods with water reflector & $\mathrm{U}(4.31 \%) \mathrm{O}_{2}$ & $\mathrm{H}_{2} \mathrm{O}$ & $\mathrm{H}_{2} \mathrm{O}$ & $\mathrm{NA}$ \\
\hline LCT-004-002 & $\mathrm{UO}_{2}$ Fuel Rods with water reflector & $\mathrm{U}(4.31 \%) \mathrm{O}_{2}$ & $\mathrm{H}_{2} \mathrm{O}$ & $\mathrm{H}_{2} \mathrm{O}$ & NA \\
\hline LCT-004-003 & $\mathrm{UO}_{2}$ Fuel Rods with water reflector & $\mathrm{U}(4.31 \%) \mathrm{O}_{2}$ & $\mathrm{H}_{2} \mathrm{O}$ & $\mathrm{H}_{2} \mathrm{O}$ & NA \\
\hline LCT-004-004 & $\mathrm{UO}_{2}$ Fuel Rods with water reflector & $\mathrm{U}(4.31 \%) \mathrm{O}_{2}$ & $\mathrm{H}_{2} \mathrm{O}$ & $\mathrm{H}_{2} \mathrm{O}$ & $\mathrm{NA}$ \\
\hline LCT-004-005 & $\mathrm{UO}_{2}$ Fuel Rods with water reflector & $\mathrm{U}(4.31 \%) \mathrm{O}_{2}$ & $\mathrm{H}_{2} \mathrm{O}$ & $\mathrm{H}_{2} \mathrm{O}$ & NA \\
\hline LCT-004-006 & $\mathrm{UO}_{2}$ Fuel Rods with water reflector & $\mathrm{U}(4.31 \%) \mathrm{O}_{2}$ & $\mathrm{H}_{2} \mathrm{O}$ & $\mathrm{H}_{2} \mathrm{O}$ & $\mathrm{NA}$ \\
\hline LCT-004-007 & $\mathrm{UO}_{2}$ Fuel Rods with water reflector & $\mathrm{U}(4.31 \%) \mathrm{O}_{2}$ & $\mathrm{H}_{2} \mathrm{O}$ & $\mathrm{H}_{2} \mathrm{O}$ & $\mathrm{NA}$ \\
\hline LCT-004-008 & $\mathrm{UO}_{2}$ Fuel Rods with water reflector & $\mathrm{U}(4.31 \%) \mathrm{O}_{2}$ & $\mathrm{H}_{2} \mathrm{O}$ & $\mathrm{BeO}$ & $\mathrm{NA}$ \\
\hline LCT-004-009 & $\mathrm{UO}_{2}$ Fuel Rods with water reflector & $\mathrm{U}(4.31 \%) \mathrm{O}_{2}$ & $\mathrm{H}_{2} \mathrm{O}$ & $\mathrm{H}_{2} \mathrm{O}$ & NA \\
\hline LCT-004-010 & $\mathrm{UO}_{2}$ Fuel Rods with water reflector & $\mathrm{U}(4.31 \%) \mathrm{O}_{2}$ & $\mathrm{H}_{2} \mathrm{O}$ & $\mathrm{H}_{2} \mathrm{O}$ & $\mathrm{NA}$ \\
\hline LCT-004-011 & $\mathrm{UO}_{2}$ Fuel Rods with water reflector & $\mathrm{U}(4.31 \%) \mathrm{O}_{2}$ & $\mathrm{H}_{2} \mathrm{O}$ & $\mathrm{H}_{2} \mathrm{O}$ & $\mathrm{NA}$ \\
\hline LCT-004-012 & $\mathrm{UO}_{2}$ Fuel Rods with water reflector & $\mathrm{U}(4.31 \%) \mathrm{O}_{2}$ & $\mathrm{H}_{2} \mathrm{O}$ & $\mathrm{H}_{2} \mathrm{O}$ & $\mathrm{NA}$ \\
\hline LCT-004-013 & $\mathrm{UO}_{2}$ Fuel Rods with water reflector & $\mathrm{U}(4.31 \%) \mathrm{O}_{2}$ & $\mathrm{H}_{2} \mathrm{O}$ & $\mathrm{H}_{2} \mathrm{O}$ & $\mathrm{NA}$ \\
\hline LCT-004-014 & $\mathrm{UO}_{2}$ Fuel Rods with water reflector & $\mathrm{U}(4.31 \%) \mathrm{O}_{2}$ & $\mathrm{H}_{2} \mathrm{O}$ & $\mathrm{H}_{2} \mathrm{O}$ & $\mathrm{NA}$ \\
\hline LCT-004-015 & $\mathrm{UO}_{2}$ Fuel Rods with water reflector & $\mathrm{U}(4.31 \%) \mathrm{O}_{2}$ & $\mathrm{H}_{2} \mathrm{O}$ & $\mathrm{H}_{2} \mathrm{O}$ & NA \\
\hline LCT-004-016 & $\mathrm{UO}_{2}$ Fuel Rods with water reflector & $\mathrm{U}(4.31 \%) \mathrm{O}_{2}$ & $\mathrm{H}_{2} \mathrm{O}$ & $\mathrm{H}_{2} \mathrm{O}$ & $\mathrm{NA}$ \\
\hline LCT-004-017 & $\mathrm{UO}_{2}$ Fuel Rods with water reflector & $\mathrm{U}(4.31 \%) \mathrm{O}_{2}$ & $\mathrm{H}_{2} \mathrm{O}$ & $\mathrm{H}_{2} \mathrm{O}$ & $\mathrm{NA}$ \\
\hline LCT-004-018 & $\mathrm{UO}_{2}$ Fuel Rods with water reflector & $\mathrm{U}(4.31 \%) \mathrm{O}_{2}$ & $\mathrm{H}_{2} \mathrm{O}$ & $\mathrm{H}_{2} \mathrm{O}$ & $\mathrm{NA}$ \\
\hline LCT-004-019 & $\mathrm{UO}_{2}$ Fuel Rods with water reflector & $\mathrm{U}(4.31 \%) \mathrm{O}_{2}$ & $\mathrm{H}_{2} \mathrm{O}$ & $\mathrm{H}_{2} \mathrm{O}$ & $\mathrm{NA}$ \\
\hline LCT-004-020 & $\mathrm{UO}_{2}$ Fuel Rods with water reflector & $\mathrm{U}(4.31 \%) \mathrm{O}_{2}$ & $\mathrm{H}_{2} \mathrm{O}$ & $\mathrm{H}_{2} \mathrm{O}$ & $\mathrm{NA}$ \\
\hline LCT-009-001 & $\mathrm{UO}_{2}$ Fuel Rods, steel absorber & $\mathrm{U}(4.31 \%) \mathrm{O}_{2}$ & $\mathrm{H}_{2} \mathrm{O}$ & $\mathrm{H}_{2} \mathrm{O}$ & $\mathrm{NA}$ \\
\hline LCT-009-002 & $\mathrm{UO}_{2}$ Fuel Rods, steel absorber & $\mathrm{U}(4.31 \%) \mathrm{O}_{2}$ & $\mathrm{H}_{2} \mathrm{O}$ & $\mathrm{H}_{2} \mathrm{O}$ & NA \\
\hline LCT-009-003 & $\mathrm{UO}_{2}$ Fuel Rods, steel absorber & $\mathrm{U}(4.31 \%) \mathrm{O}_{2}$ & $\mathrm{H}_{2} \mathrm{O}$ & $\mathrm{H}_{2} \mathrm{O}$ & NA \\
\hline LCT-009-004 & $\mathrm{UO}_{2}$ Fuel Rods, steel absorber & $\mathrm{U}(4.31 \%) \mathrm{O}_{2}$ & $\mathrm{H}_{2} \mathrm{O}$ & $\mathrm{H}_{2} \mathrm{O}$ & $\mathrm{NA}$ \\
\hline LCT-009-005 & $\mathrm{UO}_{2}$ Fuel Rods, steel absorber & $\mathrm{U}(4.31 \%) \mathrm{O}_{2}$ & $\mathrm{H}_{2} \mathrm{O}$ & $\mathrm{H}_{2} \mathrm{O}$ & NA \\
\hline LCT-009-006 & $\mathrm{UO}_{2}$ Fuel Rods, steel absorber & $\mathrm{U}(4.31 \%) \mathrm{O}_{2}$ & $\mathrm{H}_{2} \mathrm{O}$ & $\mathrm{H}_{2} \mathrm{O}$ & $\mathrm{NA}$ \\
\hline LCT-009-007 & $\mathrm{UO}_{2}$ Fuel Rods, steel absorber & $\mathrm{U}(4.31 \%) \mathrm{O}_{2}$ & $\mathrm{H}_{2} \mathrm{O}$ & $\mathrm{H}_{2} \mathrm{O}$ & $\mathrm{NA}$ \\
\hline LCT-009-008 & $\mathrm{UO}_{2}$ Fuel Rods, steel absorber & $\mathrm{U}(4.31 \%) \mathrm{O}_{2}$ & $\mathrm{H}_{2} \mathrm{O}$ & $\mathrm{H}_{2} \mathrm{O}$ & $\mathrm{NA}$ \\
\hline LCT-009-009 & $\mathrm{UO}_{2}$ Fuel Rods, boral absorber & $\mathrm{U}(4.31 \%) \mathrm{O}_{2}$ & $\mathrm{H}_{2} \mathrm{O}$ & $\mathrm{H}_{2} \mathrm{O}$ & NA \\
\hline LCT $-009-010$ & $\mathrm{UO}_{2}$ Fuel Rods, steel absorber & $\mathrm{U}(4.31 \%) \mathrm{O}_{2}$ & $\mathrm{H}_{2} \mathrm{O}$ & $\mathrm{H}_{2} \mathrm{O}$ & $\mathrm{NA}$ \\
\hline LCT-009-011 & $\mathrm{UO}_{2}$ Fuel Rods, copper absorber & $\mathrm{U}(4.31 \%) \mathrm{O}_{2}$ & $\mathrm{H}_{2} \mathrm{O}$ & $\mathrm{H}_{2} \mathrm{O}$ & NA \\
\hline LCT-009-012 & $\mathrm{UO}_{2}$ Fuel Rod, copper absorber $\mathrm{s}$ & $\mathrm{U}(4.31 \%) \mathrm{O}_{2}$ & $\mathrm{H}_{2} \mathrm{O}$ & $\mathrm{H}_{2} \mathrm{O}$ & NA \\
\hline LCT-009-013 & $\mathrm{UO}_{2}$ Fuel Rods, copper absorber & $\mathrm{U}(4.31 \%) \mathrm{O}_{2}$ & $\mathrm{H}_{2} \mathrm{O}$ & $\mathrm{H}_{2} \mathrm{O}$ & $\mathrm{NA}$ \\
\hline LCT-009-014 & $\mathrm{UO}_{2}$ Fuel Rods, copper absorber & $\mathrm{U}(4.31 \%) \mathrm{O}_{2}$ & $\mathrm{H}_{2} \mathrm{O}$ & $\mathrm{H}_{2} \mathrm{O}$ & NA \\
\hline
\end{tabular}




\begin{tabular}{|c|c|c|c|c|c|}
\hline Case Name & Geometry & Form & Moderator & Reflector & $\mathbf{H} / \mathrm{U}$ \\
\hline LCT-009-015 & $\mathrm{UO}_{2}$ Fuel Rods, copper absorber & $\mathrm{U}(4.31 \%) \mathrm{O}_{2}$ & $\mathrm{H}_{2} \mathrm{O}$ & $\mathrm{H}_{2} \mathrm{O}$ & NA \\
\hline LCT-009-016 & $\mathrm{UO}_{2}$ Fuel Rods, cadmium absorber & $\mathrm{U}(4.31 \%) \mathrm{O}_{2}$ & $\mathrm{H}_{2} \mathrm{O}$ & $\mathrm{H}_{2} \mathrm{O}$ & NA \\
\hline LCT-009-017 & $\mathrm{UO}_{2}$ Fuel Rods, cadmium absorber & $\mathrm{U}(4.31 \%) \mathrm{O}_{2}$ & $\mathrm{H}_{2} \mathrm{O}$ & $\mathrm{H}_{2} \mathrm{O}$ & NA \\
\hline LCT-009-018 & $\mathrm{UO}_{2}$ Fuel Rods, cadmium absorber & $\mathrm{U}(4.31 \%) \mathrm{O}_{2}$ & $\mathrm{H}_{2} \mathrm{O}$ & $\mathrm{H}_{2} \mathrm{O}$ & NA \\
\hline LCT-009-019 & $\mathrm{UO}_{2}$ Fuel Rods, cadmium absorber & $\mathrm{U}(4.31 \%) \mathrm{O}_{2}$ & $\mathrm{H}_{2} \mathrm{O}$ & $\mathrm{H}_{2} \mathrm{O}$ & NA \\
\hline LCT-009-020 & $\mathrm{UO}_{2}$ Fuel Rods, cadmium absorber & $\mathrm{U}(4.31 \%) \mathrm{O}_{2}$ & $\mathrm{H}_{2} \mathrm{O}$ & $\mathrm{H}_{2} \mathrm{O}$ & $\mathrm{NA}$ \\
\hline LCT-009-021 & $\mathrm{UO}_{2}$ Fuel Rods, cadmium absorber & $\mathrm{U}(4.31 \%) \mathrm{O}_{2}$ & $\mathrm{H}_{2} \mathrm{O}$ & $\mathrm{H}_{2} \mathrm{O}$ & NA \\
\hline LCT-009-022 & $\mathrm{UO}_{2}$ Fuel Rods, cadmium absorber & $\mathrm{U}(4.31 \%) \mathrm{O}_{2}$ & $\mathrm{H}_{2} \mathrm{O}$ & $\mathrm{H}_{2} \mathrm{O}$ & NA \\
\hline LCT-009-023 & $\mathrm{UO}_{2}$ Fuel Rods, cadmium absorber & $\mathrm{U}(4.31 \%) \mathrm{O}_{2}$ & $\mathrm{H}_{2} \mathrm{O}$ & $\mathrm{H}_{2} \mathrm{O}$ & NA \\
\hline LCT-009-024 & $\mathrm{UO}_{2}$ Fuel Rods, cadmium absorber & $\mathrm{U}(4.31 \%) \mathrm{O}_{2}$ & $\mathrm{H}_{2} \mathrm{O}$ & $\mathrm{H}_{2} \mathrm{O}$ & NA \\
\hline LCT-009-025 & $\mathrm{UO}_{2}$ Fuel Rods, cadmium absorber & $\mathrm{U}(4.31 \%) \mathrm{O}_{2}$ & $\mathrm{H}_{2} \mathrm{O}$ & $\mathrm{H}_{2} \mathrm{O}$ & $\mathrm{NA}$ \\
\hline LCT-009-026 & $\mathrm{UO}_{2}$ Fuel Rods, zircaloy-4 absorber & $\mathrm{U}(4.31 \%) \mathrm{O}_{2}$ & $\mathrm{H}_{2} \mathrm{O}$ & $\mathrm{H}_{2} \mathrm{O}$ & NA \\
\hline LCT-009-027 & $\mathrm{UO}_{2}$ Fuel Rods, zircaloy-4 absorber & $\mathrm{U}(4.31 \%) \mathrm{O}_{2}$ & $\mathrm{H}_{2} \mathrm{O}$ & $\mathrm{H}_{2} \mathrm{O}$ & NA \\
\hline LCT-010-001 & $\mathrm{UO}_{2}$ Fuel Rods, Lead Reflector & $\mathrm{U}(4.31 \%) \mathrm{O}_{2}$ & $\mathrm{H}_{2} \mathrm{O}$ & $\mathrm{H}_{2} \mathrm{O}$ & NA \\
\hline LCT-010-002 & $\mathrm{UO}_{2}$ Fuel Rods, Lead Reflector & $\mathrm{U}(4.31 \%) \mathrm{O}_{2}$ & $\mathrm{H}_{2} \mathrm{O}$ & $\mathrm{H}_{2} \mathrm{O}$ & NA \\
\hline LCT $-010-003$ & $\mathrm{UO}_{2}$ Fuel Rods, Lead Reflector & $\mathrm{U}(4.31 \%) \mathrm{O}_{2}$ & $\mathrm{H}_{2} \mathrm{O}$ & $\mathrm{H}_{2} \mathrm{O}$ & NA \\
\hline LCT-010-004 & $\mathrm{UO}_{2}$ Fuel Rods, Lead Reflector & $\mathrm{U}(4.31 \%) \mathrm{O}_{2}$ & $\mathrm{H}_{2} \mathrm{O}$ & $\mathrm{H}_{2} \mathrm{O}$ & NA \\
\hline LCT-010-005 & $\mathrm{UO}_{2}$ Fuel Rods, Uranium Reflector & $\mathrm{U}(4.31 \%) \mathrm{O}_{2}$ & $\mathrm{H}_{2} \mathrm{O}$ & $\mathrm{H}_{2} \mathrm{O}$ & NA \\
\hline LCT-010-006 & $\mathrm{UO}_{2}$ Fuel Rods, Uranium Reflector & $\mathrm{U}(4.31 \%) \mathrm{O}_{2}$ & $\mathrm{H}_{2} \mathrm{O}$ & $\mathrm{H}_{2} \mathrm{O}$ & NA \\
\hline LCT-010-007 & $\mathrm{UO}_{2}$ Fuel Rods, Uranium Reflector & $\mathrm{U}(4.31 \%) \mathrm{O}_{2}$ & $\mathrm{H}_{2} \mathrm{O}$ & $\mathrm{H}_{2} \mathrm{O}$ & $\overline{N A}$ \\
\hline LCT $-010-008$ & $\mathrm{UO}_{2}$ Fuel Rods, Uranium Reflector & $\mathrm{U}(4.31 \%) \mathrm{O}_{2}$ & $\mathrm{H}_{2} \mathrm{O}$ & $\mathrm{H}_{2} \mathrm{O}$ & NA \\
\hline LCT-010-009 & $\mathrm{UO}_{2}$ Fuel Rods, Steel Reflector & $\mathrm{U}(4.31 \%) \mathrm{O}_{2}$ & $\mathrm{H}_{2} \mathrm{O}$ & $\mathrm{H}_{2} \mathrm{O}$ & $\mathrm{NA}$ \\
\hline LCT-010-010 & $\mathrm{UO}_{2}$ Fuel Rods, Steel Reflector & $\mathrm{U}(4.31 \%) \mathrm{O}_{2}$ & $\mathrm{H}_{2} \mathrm{O}$ & $\mathrm{H}_{2} \mathrm{O}$ & NA \\
\hline LCT-010-011 & $\mathrm{UO}_{2}$ Fuel Rods, Steel Reflector & $\mathrm{U}(4.31 \%) \mathrm{O}_{2}$ & $\mathrm{H}_{2} \mathrm{O}$ & $\mathrm{H}_{2} \mathrm{O}$ & $\overline{N A}$ \\
\hline LCT-010-012 & $\mathrm{UO}_{2}$ Fuel Rods, Steel Reflector & $\mathrm{U}(4.31 \%) \mathrm{O}_{2}$ & $\mathrm{H}_{2} \mathrm{O}$ & $\mathrm{H}_{2} \mathrm{O}$ & NA \\
\hline LCT-010-013 & $\mathrm{UO}_{2}$ Fuel Rods, Steel Reflector & $\mathrm{U}(4.31 \%) \mathrm{O}_{2}$ & $\mathrm{H}_{2} \mathrm{O}$ & $\mathrm{H}_{2} \mathrm{O}$ & NA \\
\hline LCT-010-014 & $\mathrm{UO}_{2}$ Fuel Rods, Steel Reflector & $\mathrm{U}(4.31 \%) \mathrm{O}_{2}$ & $\mathrm{H}_{2} \mathrm{O}$ & $\mathrm{H}_{2} \mathrm{O}$ & NA \\
\hline LCT-010-015 & $\mathrm{UO}_{2}$ Fuel Rods, Steel Reflector & $\mathrm{U}(4.31 \%) \mathrm{O}_{2}$ & $\mathrm{H}_{2} \mathrm{O}$ & $\mathrm{H}_{2} \mathrm{O}$ & NA \\
\hline LCT-010-016 & $\mathrm{UO}_{2}$ Fuel Rods, Steel Reflector & $\mathrm{U}(4.31 \%) \mathrm{O}_{2}$ & $\mathrm{H}_{2} \mathrm{O}$ & $\mathrm{H}_{2} \mathrm{O}$ & NA \\
\hline LCT-010-017 & $\mathrm{UO}_{2}$ Fuel Rods, Steel Reflector & $\mathrm{U}(4.31 \%) \mathrm{O}_{2}$ & $\mathrm{H}_{2} \mathrm{O}$ & $\mathrm{H}_{2} \mathrm{O}$ & NA \\
\hline LCT-010-018 & $\mathrm{UO}_{2}$ Fuel Rods, Steel Reflector & $\mathrm{U}(4.31 \%) \mathrm{O}_{2}$ & $\mathrm{H}_{2} \mathrm{O}$ & $\mathrm{H}_{2} \mathrm{O}$ & NA \\
\hline LCT-010-019 & $\mathrm{UO}_{2}$ Fuel Rods, Steel Reflector & $\mathrm{U}(4.31 \%) \mathrm{O}_{2}$ & $\mathrm{H}_{2} \mathrm{O}$ & $\mathrm{H}_{2} \mathrm{O}$ & $\mathrm{NA}$ \\
\hline LCT-010-020 & $\mathrm{UO}_{2}$ Fuel Rods, Lead Reflector & $\mathrm{U}(4.31 \%) \mathrm{O}_{2}$ & $\mathrm{H}_{2} \mathrm{O}$ & $\mathrm{H}_{2} \mathrm{O}$ & NA \\
\hline LCT-010-021 & $\mathrm{UO}_{2}$ Fuel Rods, Lead Reflector & $\mathrm{U}(4.31 \%) \mathrm{O}_{2}$ & $\mathrm{H}_{2} \mathrm{O}$ & $\mathrm{H}_{2} \mathrm{O}$ & $\mathrm{NA}$ \\
\hline LCT-010-022 & $\mathrm{UO}_{2}$ Fuel Rods, Lead Reflector & $\mathrm{U}(4.31 \%) \mathrm{O}_{2}$ & $\mathrm{H}_{2} \mathrm{O}$ & $\mathrm{H}_{2} \mathrm{O}$ & NA \\
\hline LCT-010-023 & $\mathrm{UO}_{2}$ Fuel Rods, Lead Reflector & $\mathrm{U}(4.31 \%) \mathrm{O}_{2}$ & $\mathrm{H}_{2} \mathrm{O}$ & $\mathrm{H}_{2} \mathrm{O}$ & NA \\
\hline LCT-010-024 & $\mathrm{UO}_{2}$ Fuel Rods, Uranium Reflector & $\mathrm{U}(4.31 \%) \mathrm{O}_{2}$ & $\mathrm{H}_{2} \mathrm{O}$ & $\mathrm{H}_{2} \mathrm{O}$ & NA \\
\hline LCT-010-025 & $\mathrm{UO}_{2}$ Fuel Rods, Uranium Reflector & $\mathrm{U}(4.31 \%) \mathrm{O}_{2}$ & $\mathrm{H}_{2} \mathrm{O}$ & $\mathrm{H}_{2} \mathrm{O}$ & NA \\
\hline LCT-010-026 & $\mathrm{UO}_{2}$ Fuel Rods, Uranium Reflector & $\mathrm{U}(4.31 \%) \mathrm{O}_{2}$ & $\mathrm{H}_{2} \mathrm{O}$ & $\mathrm{H}_{2} \mathrm{O}$ & NA \\
\hline LCT-010-027 & $\mathrm{UO}_{2}$ Fuel Rods, Uranium Reflector & $\mathrm{U}(4.31 \%) \mathrm{O}_{2}$ & $\mathrm{H}_{2} \mathrm{O}$ & $\mathrm{H}_{2} \mathrm{O}$ & NA \\
\hline LCT-010-028 & $\mathrm{UO}_{2}$ Fuel Rods, Uranium Reflector & $\mathrm{U}(4.31 \%) \mathrm{O}_{2}$ & $\mathrm{H}_{2} \mathrm{O}$ & $\mathrm{H}_{2} \mathrm{O}$ & NA \\
\hline LCT-010-029 & $\mathrm{UO}_{2}$ Fuel Rods, Uranium Reflector & $\mathrm{U}(4.31 \%) \mathrm{O}_{2}$ & $\mathrm{H}_{2} \mathrm{O}$ & $\mathrm{H}_{2} \mathrm{O}$ & NA \\
\hline LCT-010-030 & $\mathrm{UO}_{2}$ Fuel Rods, Uranium Reflector & $\mathrm{U}(4.31 \%) \mathrm{O}_{2}$ & $\mathrm{H}_{2} \mathrm{O}$ & $\mathrm{H}_{2} \mathrm{O}$ & NA \\
\hline LCT-026-001 & $\mathrm{UO}_{2}$ HEX Array Fuel, $\mathrm{T}=20^{\circ} \mathrm{C}$ & $\mathrm{U}(4.92 \%) \mathrm{O}_{2}$ & $\mathrm{H}_{2} \mathrm{O}$ & $\mathrm{H}_{2} \mathrm{O}$ & $\mathrm{NA}$ \\
\hline LCT-026-002 & $\mathrm{UO}_{2} \mathrm{HEX}$ Array Fuel, $\mathrm{T}=231^{\circ} \mathrm{C}$ & $\mathrm{U}(4.92 \%) \mathrm{O}_{2}$ & $\mathrm{H}_{2} \mathrm{O}$ & $\mathrm{H}_{2} \mathrm{O}$ & NA \\
\hline LCT-026-003 & $\mathrm{UO}_{2}$ HEX Array Fuel, $\mathrm{T}=20^{\circ} \mathrm{C}$ & $\mathrm{U}(4.92 \%) \mathrm{O}_{2}$ & $\mathrm{H}_{2} \mathrm{O}$ & $\mathrm{H}_{2} \mathrm{O}$ & NA \\
\hline LCT-026-004 & $\mathrm{UO}_{2}$ HEX Array Fuel, $\mathrm{T}=206^{\circ} \mathrm{C}$ & $\mathrm{U}(4.92 \%) \mathrm{O}_{2}$ & $\mathrm{H}_{2} \mathrm{O}$ & $\mathrm{H}_{2} \mathrm{O}$ & NA \\
\hline LCT-026-005 & $\mathrm{UO}_{2} \mathrm{HEX}$ Array Fuel, $\mathrm{T}=20^{\circ} \mathrm{C}$ & $\mathrm{U}(4.92 \%) \mathrm{O}_{2}$ & $\mathrm{H}_{2} \mathrm{O}$ & $\mathrm{H}_{2} \mathrm{O}$ & NA \\
\hline
\end{tabular}




\begin{tabular}{|c|c|c|c|c|c|}
\hline Case Name & Geometry & Form & Moderator & Reflector & $\mathbf{H} / \mathrm{U}$ \\
\hline LCT-026-006 & $\mathrm{UO}_{2}$ HEX Array Fuel, $\mathrm{T}=212^{\circ} \mathrm{C}$ & $\mathrm{U}(4.92 \%) \mathrm{O}_{2}$ & $\mathrm{H}_{2} \mathrm{O}$ & $\mathrm{H}_{2} \mathrm{O}$ & NA \\
\hline LCT-042-001 & $\mathrm{UO}_{2}$ Fuel Rods, 304-L, Steel Reflector & $\mathrm{U}(2.35 \%) \mathrm{O}_{2}$ & $\mathrm{H}_{2} \mathrm{O}$ & Stainless Steel & NA \\
\hline LCT-042-002 & $\mathrm{UO}_{2}$ Fuel Rods, 304-L, Steel Reflector & $\mathrm{U}(2.35 \%) \mathrm{O}_{2}$ & $\mathrm{H}_{2} \mathrm{O}$ & Stainless Steel & NA \\
\hline LCT-042-003 & $\mathrm{UO}_{2}$ Fuel Rods, 304-L, Steel Reflector & $\mathrm{U}(2.35 \%) \mathrm{O}_{2}$ & $\mathrm{H}_{2} \mathrm{O}$ & Stainless Steel & NA \\
\hline LCT-042-004 & $\mathrm{UO}_{2}$ Fuel Rods, 304-L, Steel Reflector & $\mathrm{U}(2.35 \%) \mathrm{O}_{2}$ & $\mathrm{H}_{2} \mathrm{O}$ & Stainless Steel & NA \\
\hline LCT-042-005 & $\mathrm{UO}_{2}$ Fuel Rods, 304-L, Steel Reflector & $\mathrm{U}(2.35 \%) \mathrm{O}_{2}$ & $\mathrm{H}_{2} \mathrm{O}$ & Stainless Steel & NA \\
\hline LCT-042-006 & $\mathrm{UO}_{2}$ Fuel Rods, 304-L, Steel Reflector & $\mathrm{U}(2.35 \%) \mathrm{O}_{2}$ & $\mathrm{H}_{2} \mathrm{O}$ & Stainless Steel & NA \\
\hline LCT-042-007 & $\mathrm{UO}_{2}$ Fuel Rods, 304-L, Steel Reflector & $\mathrm{U}(2.35 \%) \mathrm{O}_{2}$ & $\mathrm{H}_{2} \mathrm{O}$ & Stainless Steel & $\mathrm{NA}$ \\
\hline LCT-049-001 & $\mathrm{UO}_{2}$ Powder, Polyethylene Reflector & $\mathrm{U}(5.0 \%) \mathrm{O}_{2}$ & $\mathrm{H}_{2} \mathrm{O}$ & Polyethylene & 2.0 \\
\hline LCT-049-002 & $\mathrm{UO}_{2}$ Powder, Polyethylene Reflector & $\mathrm{U}(5.0 \%) \mathrm{O}_{2}$ & $\mathrm{H}_{2} \mathrm{O}$ & Polyethylene & 2.0 \\
\hline LCT-049-003 & $\mathrm{UO}_{2}$ Powder, Polyethylene Reflector & $\mathrm{U}(5.0 \%) \mathrm{O}_{2}$ & $\mathrm{H}_{2} \mathrm{O}$ & Polyethylene & 2.0 \\
\hline LCT-049-004 & $\mathrm{UO}_{2}$ Powder, Polyethylene Reflector & $\mathrm{U}(5.0 \%) \mathrm{O}_{2}$ & $\mathrm{H}_{2} \mathrm{O}$ & Polyethylene & 2.0 \\
\hline LCT-049-005 & $\mathrm{UO}_{2}$ Powder, Polyethylene Reflector & $\mathrm{U}(5.0 \%) \mathrm{O}_{2}$ & $\mathrm{H}_{2} \mathrm{O}$ & Polyethylene & 2.5 \\
\hline LCT-049-006 & $\mathrm{UO}_{2}$ Powder, Polyethylene Reflector & $\mathrm{U}(5.0 \%) \mathrm{O}_{2}$ & $\mathrm{H}_{2} \mathrm{O}$ & Polyethylene & 2.5 \\
\hline LCT-049-007 & $\mathrm{UO}_{2}$ Powder, Polyethylene Reflector & $\mathrm{U}(5.0 \%) \mathrm{O}_{2}$ & $\mathrm{H}_{2} \mathrm{O}$ & Polyethylene & 2.5 \\
\hline LCT-049-008 & $\mathrm{UO}_{2}$ Powder, Polyethylene Reflector & $\mathrm{U}(5.0 \%) \mathrm{O}_{2}$ & $\mathrm{H}_{2} \mathrm{O}$ & Polyethylene & 2.5 \\
\hline LCT-049-009 & $\mathrm{UO}_{2}$ Powder, Polyethylene Reflector & $\mathrm{U}(5.0 \%) \mathrm{O}_{2}$ & $\mathrm{H}_{2} \mathrm{O}$ & Polyethylene & 3.0 \\
\hline LCT-049-010 & $\mathrm{UO}_{2}$ Powder, Polyethylene Reflector & $\mathrm{U}(5.0 \%) \mathrm{O}_{2}$ & $\mathrm{H}_{2} \mathrm{O}$ & Polyethylene & 3.0 \\
\hline LCT-049-011 & $\mathrm{UO}_{2}$ Powder, Polyethylene Reflector & $\mathrm{U}(5.0 \%) \mathrm{O}_{2}$ & $\mathrm{H}_{2} \mathrm{O}$ & Polyethylene & 3.0 \\
\hline LCT-049-012 & $\mathrm{UO}_{2}$ Powder, Polyethylene Reflector & $\mathrm{U}(5.0 \%) \mathrm{O}_{2}$ & $\mathrm{H}_{2} \mathrm{O}$ & Polyethylene & 3.0 \\
\hline LCT-049-013 & $\mathrm{UO}_{2}$ Powder, Polyethylene Reflector & $\mathrm{U}(5.0 \%) \mathrm{O}_{2}$ & $\mathrm{H}_{2} \mathrm{O}$ & Polyethylene & mixed \\
\hline LCT-049-014 & $\mathrm{UO}_{2}$ Powder, Polyethylene Reflector & $\mathrm{U}(5.0 \%) \mathrm{O}_{2}$ & $\mathrm{H}_{2} \mathrm{O}$ & Polyethylene & mixed \\
\hline LCT-049-015 & $\mathrm{UO}_{2}$ Powder, Polyethylene Reflector & $\mathrm{U}(5.0 \%) \mathrm{O}_{2}$ & $\mathrm{H}_{2} \mathrm{O}$ & Polyethylene & mixed \\
\hline LCT-049-016 & $\mathrm{UO}_{2}$ Powder, Polyethylene Reflector & $\mathrm{U}(5.0 \%) \mathrm{O}_{2}$ & $\mathrm{H}_{2} \mathrm{O}$ & Polyethylene & mixed \\
\hline LCT-049-017 & $\mathrm{UO}_{2}$ Powder, Polyethylene Reflector & $\mathrm{U}(5.0 \%) \mathrm{O}_{2}$ & $\mathrm{H}_{2} \mathrm{O}$ & Polyethylene & mixed \\
\hline LCT-049-018 & $\mathrm{UO}_{2}$ Powder, Polyethylene Reflector & $\mathrm{U}(5.0 \%) \mathrm{O}_{2}$ & $\mathrm{H}_{2} \mathrm{O}$ & Polyethylene & mixed \\
\hline LCT-052-001 & $\mathrm{UO}_{2}$ HEX Array Fuel & $\mathrm{U}(4.738 \%) \mathrm{O}_{2}$ & $\mathrm{H}_{2} \mathrm{O}$ & $\mathrm{Gd}\left(\mathrm{NO}_{3}\right)_{3}$ & NA \\
\hline LCT-052-002 & $\mathrm{UO}_{2}$ HEX Array Fuel & $\mathrm{U}(4.738 \%) \mathrm{O}_{2}$ & $\mathrm{H}_{2} \mathrm{O}$ & $\mathrm{Gd}\left(\mathrm{NO}_{3}\right)_{3}$ & NA \\
\hline LCT-052-003 & $\mathrm{UO}_{2}$ HEX Array Fuel & $\mathrm{U}(4.738 \%) \mathrm{O}_{2}$ & $\mathrm{H}_{2} \mathrm{O}$ & $\mathrm{Gd}\left(\mathrm{NO}_{3}\right)_{3}$ & NA \\
\hline LCT-052-004 & $\mathrm{UO}_{2} \mathrm{HEX}$ Array Fuel & $\mathrm{U}(4.738 \%) \mathrm{O}_{2}$ & $\mathrm{H}_{2} \mathrm{O}$ & $\mathrm{Gd}\left(\mathrm{NO}_{3}\right)_{3}$ & NA \\
\hline LCT-052-005 & $\mathrm{UO}_{2}$ HEX Array Fuel & $\mathrm{U}(4.738 \%) \mathrm{O}_{2}$ & $\mathrm{H}_{2} \mathrm{O}$ & $\mathrm{Gd}\left(\mathrm{NO}_{3}\right)_{3}$ & NA \\
\hline LCT-052-006 & $\mathrm{UO}_{2}$ HEX Array Fuel & $\mathrm{U}(4.738 \%) \mathrm{O}_{2}$ & $\mathrm{H}_{2} \mathrm{O}$ & $\mathrm{Gd}\left(\mathrm{NO}_{3}\right)_{3}$ & NA \\
\hline LST-003-001 & $32.95 \mathrm{~cm}$ sphere, $126.3 \mathrm{~L} .296 \mathrm{~g} \mathrm{U} / \mathrm{L}$ & $\mathrm{U}(10 \%) \mathrm{O}_{2}\left(\mathrm{NO}_{3}\right)_{2}$ & $\mathrm{H}_{2} \mathrm{O}$ & Bare & 770 \\
\hline LST-003-002 & $32.95 \mathrm{~cm}$ sphere, 144.1 L. $264 \mathrm{~g} \mathrm{U} / \mathrm{L}$ & $\mathrm{U}(10 \%) \mathrm{O}_{2}\left(\mathrm{NO}_{3}\right)_{2}$ & $\mathrm{H}_{2} \mathrm{O}$ & Bare & 878 \\
\hline LST-003-003 & $32.95 \mathrm{~cm}$ sphere, $149.9 \mathrm{~L} .260 \mathrm{~g} \mathrm{U} / \mathrm{L}$ & $\mathrm{U}(10 \%) \mathrm{O}_{2}\left(\mathrm{NO}_{3}\right)_{2}$ & $\mathrm{H}_{2} \mathrm{O}$ & Bare & 897 \\
\hline LST-003-004 & $43.63 \mathrm{~cm}$ sphere, $197.6 \mathrm{~L} .255 \mathrm{~g} \mathrm{U} / \mathrm{L}$ & $\mathrm{U}(10 \%) \mathrm{O}_{2}\left(\mathrm{NO}_{3}\right)_{2}$ & $\mathrm{H}_{2} \mathrm{O}$ & Bare & 913 \\
\hline LST-003-005 & $43.63 \mathrm{~cm}$ sphere, $318.6 \mathrm{~L} .203 \mathrm{~g} \mathrm{U} / \mathrm{L}$ & $\mathrm{U}(10 \%) \mathrm{O}_{2}\left(\mathrm{NO}_{3}\right)_{2}$ & $\mathrm{H}_{2} \mathrm{O}$ & Bare & 1173 \\
\hline LST-003-006 & $43.63 \mathrm{~cm}$ sphere, $347.9 \mathrm{~L} .197 \mathrm{~g} \mathrm{U} / \mathrm{L}$ & $\mathrm{U}(10 \%) \mathrm{O}_{2}\left(\mathrm{NO}_{3}\right)_{2}$ & $\mathrm{H}_{2} \mathrm{O}$ & Bare & 1213 \\
\hline LST-003-007 & $59.89 \mathrm{~cm}$ sphere, $501.8 \mathrm{~L} .193 \mathrm{~g} \mathrm{U} / \mathrm{L}$ & $\mathrm{U}(10 \%) \mathrm{O}_{2}\left(\mathrm{NO}_{3}\right)_{2}$ & $\mathrm{H}_{2} \mathrm{O}$ & Bare & 1240 \\
\hline LST-003-008 & $59.89 \mathrm{~cm}$ sphere, $845.2 \mathrm{~L} .171 \mathrm{~g} \mathrm{U} / \mathrm{L}$ & $\mathrm{U}(10 \%) \mathrm{O}_{2}\left(\mathrm{NO}_{3}\right)_{2}$ & $\mathrm{H}_{2} \mathrm{O}$ & Bare & 1412 \\
\hline LST-003-009 & $59.89 \mathrm{~cm}$ sphere, $899.8 \mathrm{~L} .168 \mathrm{~g} \mathrm{U} / \mathrm{L}$ & $\mathrm{U}(10 \%) \mathrm{O}_{2}\left(\mathrm{NO}_{3}\right)_{2}$ & $\mathrm{H}_{2} \mathrm{O}$ & Bare & 1438 \\
\hline LST-004-001 & $60 \mathrm{~cm}$ ID cylinder tank, $310 \mathrm{~g} \mathrm{U} / \mathrm{L}$ & $\mathrm{U}(10 \%) \mathrm{O}_{2}\left(\mathrm{NO}_{3}\right)_{2}$ & $\mathrm{H}_{2} \mathrm{O}$ & $\mathrm{H}_{2} \mathrm{O}$ & 719 \\
\hline LST-004-029 & $60 \mathrm{~cm}$ ID cylinder tank, $290 \mathrm{~g} \mathrm{U} / \mathrm{L}$ & $\mathrm{U}(10 \%) \mathrm{O}_{2}\left(\mathrm{NO}_{3}\right)_{2}$ & $\mathrm{H}_{2} \mathrm{O}$ & $\mathrm{H}_{2} \mathrm{O}$ & 771 \\
\hline LST-004-033 & $60 \mathrm{~cm}$ ID cylinder tank, $270 \mathrm{~g} \mathrm{U} / \mathrm{L}$ & $\mathrm{U}(10 \%) \mathrm{O}_{2}\left(\mathrm{NO}_{3}\right)_{2}$ & $\mathrm{H}_{2} \mathrm{O}$ & $\mathrm{H}_{2} \mathrm{O}$ & 842 \\
\hline LST-004-034 & $60 \mathrm{~cm}$ ID cylinder tank, $254 \mathrm{~g} \mathrm{U} / \mathrm{L}$ & $\mathrm{U}(10 \%) \mathrm{O}_{2}\left(\mathrm{NO}_{3}\right)_{2}$ & $\mathrm{H}_{2} \mathrm{O}$ & $\mathrm{H}_{2} \mathrm{O}$ & 896 \\
\hline
\end{tabular}




\begin{tabular}{|c|c|c|c|c|c|}
\hline Case Name & Geometry & Form & Moderator & Reflector & $\mathrm{H} / \mathrm{U}$ \\
\hline LST-004-046 & $60 \mathrm{~cm}$ ID cylinder tank, $242 \mathrm{~g} \mathrm{U} / \mathrm{L}$ & $\mathrm{U}(10 \%) \mathrm{O}_{2}\left(\mathrm{NO}_{3}\right)_{2}$ & $\mathrm{H}_{2} \mathrm{O}$ & $\mathrm{H}_{2} \mathrm{O}$ & 942 \\
\hline LST-004-051 & $60 \mathrm{~cm}$ ID cylinder tank, $233 \mathrm{~g} \mathrm{U} / \mathrm{L}$ & $\mathrm{U}(10 \%) \mathrm{O}_{2}\left(\mathrm{NO}_{3}\right)_{2}$ & $\mathrm{H}_{2} \mathrm{O}$ & $\mathrm{H}_{2} \mathrm{O}$ & 983 \\
\hline LST-004-054 & $60 \mathrm{~cm}$ ID cylinder tank, $226 \mathrm{~g} \mathrm{U} / \mathrm{L}$ & $\mathrm{U}(10 \%) \mathrm{O}_{2}\left(\mathrm{NO}_{3}\right)_{2}$ & $\mathrm{H}_{2} \mathrm{O}$ & $\mathrm{H}_{2} \mathrm{O}$ & 1018 \\
\hline LST-005-001 & $110 \mathrm{~cm}$ ID cylinder tank, $\mathrm{B}_{4} \mathrm{C}$ Rods & $\mathrm{U}(5.64 \%) \mathrm{O}_{2}\left(\mathrm{NO}_{3}\right)_{2}$ & $\mathrm{H}_{2} \mathrm{O}$ & $\mathrm{H}_{2} \mathrm{O}$ and $\mathrm{HNO}_{3}$ & 972 \\
\hline LST-005-002 & $110 \mathrm{~cm}$ ID cylinder tank, $\mathrm{B}_{4} \mathrm{C}$ Rods & $\mathrm{U}(5.64 \%) \mathrm{O}_{2}\left(\mathrm{NO}_{3}\right)_{2}$ & $\mathrm{H}_{2} \mathrm{O}$ & $\mathrm{H}_{2} \mathrm{O}$ and $\mathrm{HNO}_{3}$ & 972 \\
\hline LST-005-003 & $110 \mathrm{~cm}$ ID cylinder tank, $\mathrm{B}_{4} \mathrm{C}$ Rods & $\mathrm{U}(5.64 \%) \mathrm{O}_{2}\left(\mathrm{NO}_{3}\right)_{2}$ & $\mathrm{H}_{2} \mathrm{O}$ & $\mathrm{H}_{2} \mathrm{O}$ and $\mathrm{HNO}_{3}$ & 972 \\
\hline LST-006-001 & $110 \mathrm{~cm}$ ID cylinder tank, $B_{4} C$ Rods & $\mathrm{U}(10 \%) \mathrm{O}_{2}\left(\mathrm{NO}_{3}\right)_{2}$ & $\mathrm{H}_{2} \mathrm{O}$ & $\mathrm{H}_{2} \mathrm{O}$ and $\mathrm{HNO}_{3}$ & 532 \\
\hline LST-006-002 & $110 \mathrm{~cm}$ ID cylinder tank, $B_{4} C$ Rods & $\mathrm{U}(10 \%) \mathrm{O}_{2}\left(\mathrm{NO}_{3}\right)_{2}$ & $\mathrm{H}_{2} \mathrm{O}$ & $\mathrm{H}_{2} \mathrm{O}$ and $\mathrm{HNO}_{3}$ & 532 \\
\hline LST-006-003 & $110 \mathrm{~cm}$ ID cylinder tank, $\mathrm{B}_{4} \mathrm{C}$ Rods & $\mathrm{U}(10 \%) \mathrm{O}_{2}\left(\mathrm{NO}_{3}\right)_{2}$ & $\mathrm{H}_{2} \mathrm{O}$ & $\mathrm{H}_{2} \mathrm{O}$ and $\mathrm{HNO}_{3}$ & 532 \\
\hline LST-006-004 & $110 \mathrm{~cm}$ ID cylinder tank, $\mathrm{B}_{4} \mathrm{C}$ Rods & $\mathrm{U}(10 \%) \mathrm{O}_{2}\left(\mathrm{NO}_{3}\right)_{2}$ & $\mathrm{H}_{2} \mathrm{O}$ & $\mathrm{H}_{2} \mathrm{O}$ and $\mathrm{HNO}_{3}$ & 532 \\
\hline LST-006-005 & $110 \mathrm{~cm}$ ID cylinder tank, $\mathrm{B}_{4} \mathrm{C}$ Rods & $\mathrm{U}(10 \%) \mathrm{O}_{2}\left(\mathrm{NO}_{3}\right)_{2}$ & $\mathrm{H}_{2} \mathrm{O}$ & $\mathrm{H}_{2} \mathrm{O}$ and $\mathrm{HNO}_{3}$ & 532 \\
\hline LST-007-014 & $60 \mathrm{~cm}$ ID cylinder tank, $310 \mathrm{~g} \mathrm{U} / \mathrm{L}$ & $\mathrm{U}(10 \%) \mathrm{O}_{2}\left(\mathrm{NO}_{3}\right)_{2}$ & $\mathrm{H}_{2} \mathrm{O}$ & Bare & 709 \\
\hline LST-007-030 & $60 \mathrm{~cm}$ ID cylinder tank, $290 \mathrm{~g} \mathrm{U} / \mathrm{L}$ & $\mathrm{U}(10 \%) \mathrm{O}_{2}\left(\mathrm{NO}_{3}\right)_{2}$ & $\mathrm{H}_{2} \mathrm{O}$ & Bare & 770 \\
\hline LST-007-032 & $60 \mathrm{~cm}$ ID cylinder tank, $270 \mathrm{~g} \mathrm{U} / \mathrm{L}$ & $\mathrm{U}(10 \%) \mathrm{O}_{2}\left(\mathrm{NO}_{3}\right)_{2}$ & $\mathrm{H}_{2} \mathrm{O}$ & Bare & 842 \\
\hline LST-007-036 & $60 \mathrm{~cm}$ ID cylinder tank, $254 \mathrm{~g} \mathrm{U} / \mathrm{L}$ & $\mathrm{U}(10 \%) \mathrm{O}_{2}\left(\mathrm{NO}_{3}\right)_{2}$ & $\mathrm{H}_{2} \mathrm{O}$ & Bare & 896 \\
\hline LST-007-049 & $60 \mathrm{~cm}$ ID cylinder tank, $242 \mathrm{~g} \mathrm{U} / \mathrm{L}$ & $\mathrm{U}(10 \%) \mathrm{O}_{2}\left(\mathrm{NO}_{3}\right)_{2}$ & $\mathrm{H}_{2} \mathrm{O}$ & Bare & 942 \\
\hline LST-016-105 & STACY $28 \mathrm{~cm} \mathrm{Slab,} 464.4 \mathrm{~g} \mathrm{U} / \mathrm{L}$ & $\mathrm{U}(10 \%) \mathrm{O}_{2}\left(\mathrm{NO}_{3}\right)_{2}$ & $\mathrm{H}_{2} \mathrm{O}$ & Bare & 469 \\
\hline LST-016-113 & STACY $28 \mathrm{~cm}$ Slab, $429.9 \mathrm{~g} \mathrm{U} / \mathrm{L}$ & $\mathrm{U}(10 \%) \mathrm{O}_{2}\left(\mathrm{NO}_{3}\right)_{2}$ & $\mathrm{H}_{2} \mathrm{O}$ & Bare & 514 \\
\hline LST-016-125 & STACY $28 \mathrm{~cm}$ Slab, $371.9 \mathrm{~g} \mathrm{U} / \mathrm{L}$ & $\mathrm{U}(10 \%) \mathrm{O}_{2}\left(\mathrm{NO}_{3}\right)_{2}$ & $\mathrm{H}_{2} \mathrm{O}$ & Bare & 608 \\
\hline LST-016-129 & STACY $28 \mathrm{~cm} \mathrm{Slab,} 350.8 \mathrm{~g} \mathrm{U} / \mathrm{L}$ & $\mathrm{U}(10 \%) \mathrm{O}_{2}\left(\mathrm{NO}_{3}\right)_{2}$ & $\mathrm{H}_{2} \mathrm{O}$ & Bare & 650 \\
\hline LST-016-131 & STACY $28 \mathrm{~cm}$ Slab, $328.9 \mathrm{~g} \mathrm{U} / \mathrm{L}$ & $\mathrm{U}(10 \%) \mathrm{O}_{2}\left(\mathrm{NO}_{3}\right)_{2}$ & $\mathrm{H}_{2} \mathrm{O}$ & Bare & 700 \\
\hline LST-016-140 & STACY $28 \mathrm{~cm}$ Slab, $311.4 \mathrm{~g} \mathrm{U} / \mathrm{L}$ & $\mathrm{U}(10 \%) \mathrm{O}_{2}\left(\mathrm{NO}_{3}\right)_{2}$ & $\mathrm{H}_{2} \mathrm{O}$ & Bare & 739 \\
\hline LST-016-196 & STACY $28 \mathrm{~cm}$ Slab, $299.6 \mathrm{~g} \mathrm{U} / \mathrm{L}$ & $\mathrm{U}(10 \%) \mathrm{O}_{2}\left(\mathrm{NO}_{3}\right)_{2}$ & $\mathrm{H}_{2} \mathrm{O}$ & Bare & 772 \\
\hline LST-017-104 & STACY $28 \mathrm{~cm}$ Slab, $464.2 \mathrm{~g} \mathrm{U} / \mathrm{L}$ & $\mathrm{U}(10 \%) \mathrm{O}_{2}\left(\mathrm{NO}_{3}\right)_{2}$ & $\mathrm{H}_{2} \mathrm{O}$ & Bare & 469 \\
\hline LST-017-122 & STACY $28 \mathrm{~cm}$ Slab, $432.4 \mathrm{~g} \mathrm{U} / \mathrm{L}$ & $\mathrm{U}(10 \%) \mathrm{O}_{2}\left(\mathrm{NO}_{3}\right)_{2}$ & $\mathrm{H}_{2} \mathrm{O}$ & Bare & 510 \\
\hline LST-017-123 & STACY $28 \mathrm{~cm} \mathrm{Slab,} 369.7 \mathrm{~g} \mathrm{U} / \mathrm{L}$ & $\mathrm{U}(10 \%) \mathrm{O}_{2}\left(\mathrm{NO}_{3}\right)_{2}$ & $\mathrm{H}_{2} \mathrm{O}$ & Bare & 611 \\
\hline LST-017-126 & STACY $28 \mathrm{~cm} \mathrm{Slab,} 350.6 \mathrm{~g} \mathrm{U} / \mathrm{L}$ & $\mathrm{U}(10 \%) \mathrm{O}_{2}\left(\mathrm{NO}_{3}\right)_{2}$ & $\mathrm{H}_{2} \mathrm{O}$ & Bare & 650 \\
\hline LST-017-130 & STACY $28 \mathrm{~cm}$ Slab, $328.9 \mathrm{~g} \mathrm{U} / \mathrm{L}$ & $\mathrm{U}(10 \%) \mathrm{O}_{2}\left(\mathrm{NO}_{3}\right)_{2}$ & $\mathrm{H}_{2} \mathrm{O}$ & Bare & 700 \\
\hline LST-017-147 & STACY $28 \mathrm{~cm}$ Slab, $315.3 \mathrm{~g} \mathrm{U} / \mathrm{L}$ & $\mathrm{U}(10 \%) \mathrm{O}_{2}\left(\mathrm{NO}_{3}\right)_{2}$ & $\mathrm{H}_{2} \mathrm{O}$ & Bare & 729 \\
\hline
\end{tabular}

Table 2-2 below contains a list of the cases run and some reported information for each case. The information was retrieved utilizing the DICE code that is included on the September 2005 Edition of the Handbook DVD (NEA/NSC/DOC(95)03, 2005). The EALF, the volume of moderator to volume of fuel ratio $\left(\mathrm{V}_{\mathrm{m}} / \mathrm{V}_{\mathrm{f}}\right)$, the ${ }^{239} \mathrm{Pu}$ over uranium plus plutonium ratio $\left[{ }^{239} \mathrm{Pu} /(\mathrm{U}+\mathrm{Pu})\right]$ (not applicable in this validation and is replaced by the ratio of $\left.{ }^{235} \mathrm{U} / \mathrm{U}\right)$, the moderator over uranium plus plutonium ratio $\left[\mathrm{H} /(\mathrm{U}+\mathrm{Pu})\right.$, replaced by $\left.\left.\mathrm{H} /{ }^{235} \mathrm{U}\right)\right]$, and the reported benchmark $\mathrm{k}_{\text {eff }}\left(\mathrm{k}_{\text {bench }}\right)$ and $\left(\sigma_{\text {bench }}\right)$ uncertainty are all included. More detailed information for each case may be found in the benchmark handbook. 
Table 2-2. Case Name and Reported Information

\begin{tabular}{|c|c|c|c|c|c|c|}
\hline Case Name & EALF & $V_{m} / V_{f}$ & ${ }^{235} \mathrm{U} / \mathrm{U}$ & $\mathbf{H} /{ }^{235} \mathrm{U}$ & $\mathbf{k}_{\text {bench }}$ & $\sigma_{\text {bench }}$ \\
\hline ICT-002-001 & $9.13 \mathrm{E}-08$ & $7.15 \mathrm{E}+00$ & 0.1700 & N/A & 1.0014 & 0.0039 \\
\hline ICT-002-002 & $1.35 \mathrm{E}-07$ & $7.15 \mathrm{E}+00$ & 0.1700 & N/A & 1.0019 & 0.0040 \\
\hline ICT-002-003 & $9.96 \mathrm{E}-08$ & $7.15 \mathrm{E}+00$ & 0.1700 & N/A & 1.0017 & 0.0044 \\
\hline ICT-002-004 & $1.06 \mathrm{E}-07$ & $7.15 \mathrm{E}+00$ & 0.1700 & N/A & 1.0019 & 0.0044 \\
\hline ICT-002-005 & $9.92 \mathrm{E}-08$ & $7.15 \mathrm{E}+00$ & 0.1700 & N/A & 1.0014 & 0.0043 \\
\hline ICT-002-006 & $1.05 \mathrm{E}-07$ & $7.15 \mathrm{E}+00$ & 0.1700 & N/A & 1.0016 & 0.0044 \\
\hline LCT-002-001 & $1.30 \mathrm{E}-07$ & $3.88 \mathrm{E}+00$ & 0.0431 & N/A & 0.9997 & 0.0020 \\
\hline LCT-002-002 & $1.30 \mathrm{E}-07$ & $3.88 \mathrm{E}+00$ & 0.0431 & N/A & 0.9997 & 0.0020 \\
\hline LCT-002-003 & $1.30 \mathrm{E}-07$ & $3.88 \mathrm{E}+00$ & 0.0431 & N/A & 0.9997 & 0.0020 \\
\hline LCT-002-004 & $1.28 \mathrm{E}-07$ & $3.88 \mathrm{E}+00$ & 0.0431 & N/A & 0.9997 & 0.0020 \\
\hline LCT-002-005 & $1.27 \mathrm{E}-07$ & $3.88 \mathrm{E}+00$ & 0.0431 & N/A & 0.9997 & 0.0020 \\
\hline LCT-004-001 & $3.51 \mathrm{E}-07$ & $1.60 \mathrm{E}+00$ & 0.0431 & N/A & 0.9998 & 0.0033 \\
\hline LCT-004-002 & $3.52 \mathrm{E}-07$ & $1.60 \mathrm{E}+00$ & 0.0431 & N/A & 0.9998 & 0.0033 \\
\hline LCT-004-003 & $3.51 \mathrm{E}-07$ & $1.60 \mathrm{E}+00$ & 0.0431 & N/A & 0.9998 & 0.0033 \\
\hline LCT-004-004 & $3.53 \mathrm{E}-07$ & $1.60 \mathrm{E}+00$ & 0.0431 & N/A & 0.9998 & 0.0033 \\
\hline LCT-004-005 & $2.36 \mathrm{E}-07$ & $1.60 \mathrm{E}+00$ & 0.0431 & N/A & 0.9998 & 0.0033 \\
\hline LCT-004-006 & $2.82 \mathrm{E}-07$ & $1.60 \mathrm{E}+00$ & 0.0431 & N/A & 0.9998 & 0.0033 \\
\hline LCT-004-007 & $2.43 \mathrm{E}-07$ & $1.60 \mathrm{E}+00$ & 0.0431 & N/A & 0.9998 & 0.0033 \\
\hline LCT-004-008 & $2.90 \mathrm{E}-07$ & $1.60 \mathrm{E}+00$ & 0.0431 & N/A & 0.9998 & 0.0035 \\
\hline LCT-004-009 & $3.34 \mathrm{E}-07$ & $1.60 \mathrm{E}+00$ & 0.0431 & N/A & 0.9998 & 0.0035 \\
\hline LCT-004-010 & $3.26 \mathrm{E}-07$ & $1.60 \mathrm{E}+00$ & 0.0431 & N/A & 0.9998 & 0.0035 \\
\hline LCT-004-011 & $2.52 \mathrm{E}-07$ & $1.60 \mathrm{E}+00$ & 0.0431 & N/A & 0.9998 & 0.0035 \\
\hline LCT-004-012 & $2.71 \mathrm{E}-07$ & $1.60 \mathrm{E}+00$ & 0.0431 & N/A & 0.9998 & 0.0035 \\
\hline LCT-004-013 & $2.83 \mathrm{E}-07$ & $1.60 \mathrm{E}+00$ & 0.0431 & N/A & 0.9998 & 0.0035 \\
\hline LCT-004-014 & $2.81 \mathrm{E}-07$ & $1.60 \mathrm{E}+00$ & 0.0431 & N/A & 0.9998 & 0.0035 \\
\hline LCT-004-015 & $2.81 \mathrm{E}-07$ & $1.60 \mathrm{E}+00$ & 0.0431 & N/A & 0.9998 & 0.0035 \\
\hline LCT-004-016 & $2.82 \mathrm{E}-07$ & $1.60 \mathrm{E}+00$ & 0.0431 & N/A & 0.9998 & 0.0035 \\
\hline LCT-004-017 & $2.84 \mathrm{E}-07$ & $1.60 \mathrm{E}+00$ & 0.0431 & N/A & 0.9998 & 0.0035 \\
\hline LCT-004-018 & $2.89 \mathrm{E}-07$ & $1.60 \mathrm{E}+00$ & 0.0431 & N/A & 0.9998 & 0.0035 \\
\hline LCT-004-019 & $2.91 \mathrm{E}-07$ & $1.60 \mathrm{E}+00$ & 0.0431 & N/A & 0.9998 & 0.0035 \\
\hline LCT-004-020 & $2.67 \mathrm{E}-07$ & $1.60 \mathrm{E}+00$ & 0.0431 & N/A & 0.9998 & 0.0035 \\
\hline LCT-009-001 & $1.30 \mathrm{E}-07$ & $3.88 \mathrm{E}+00$ & 0.0431 & N/A & 1.0000 & 0.0021 \\
\hline LCT-009-002 & $1.29 \mathrm{E}-07$ & $3.88 \mathrm{E}+00$ & 0.0431 & N/A & 1.0000 & 0.0021 \\
\hline LCT-009-003 & $1.30 \mathrm{E}-07$ & $3.88 \mathrm{E}+00$ & 0.0431 & N/A & 1.0000 & 0.0021 \\
\hline LCT-009-004 & $1.29 \mathrm{E}-07$ & $3.88 \mathrm{E}+00$ & 0.0431 & N/A & 1.0000 & 0.0021 \\
\hline LCT-009-005 & $1.31 \mathrm{E}-07$ & $3.88 \mathrm{E}+00$ & 0.0431 & N/A & 1.0000 & 0.0021 \\
\hline LCT-009-006 & $1.30 \mathrm{E}-07$ & $3.88 \mathrm{E}+00$ & 0.0431 & N/A & 1.0000 & 0.0021 \\
\hline LCT-009-007 & $1.31 \mathrm{E}-07$ & $3.88 \mathrm{E}+00$ & 0.0431 & N/A & 1.0000 & 0.0021 \\
\hline LCT-009-008 & $1.30 \mathrm{E}-07$ & $3.88 \mathrm{E}+00$ & 0.0431 & N/A & 1.0000 & 0.0021 \\
\hline LCT-009-009 & $1.31 \mathrm{E}-07$ & $3.88 \mathrm{E}+00$ & 0.0431 & N/A & 1.0000 & 0.0021 \\
\hline LCT-009-010 & $1.30 \mathrm{E}-07$ & $3.88 \mathrm{E}+00$ & 0.0431 & N/A & 1.0000 & 0.0021 \\
\hline LCT-009-011 & $1.29 \mathrm{E}-07$ & $3.88 \mathrm{E}+00$ & 0.0431 & N/A & 1.0000 & 0.0021 \\
\hline LCT-009-012 & $1.30 \mathrm{E}-07$ & $3.88 \mathrm{E}+00$ & 0.0431 & N/A & 1.0000 & 0.0021 \\
\hline LCT-009-013 & $1.29 \mathrm{E}-07$ & $3.88 \mathrm{E}+00$ & 0.0431 & N/A & 1.0000 & 0.0021 \\
\hline LCT-009-014 & $1.31 \mathrm{E}-07$ & $3.88 \mathrm{E}+00$ & 0.0431 & N/A & 1.0000 & 0.0021 \\
\hline LCT-009-015 & $1.30 \mathrm{E}-07$ & $3.88 \mathrm{E}+00$ & 0.0431 & N/A & 1.0000 & 0.0021 \\
\hline LCT-009-016 & $1.31 \mathrm{E}-07$ & $3.88 \mathrm{E}+00$ & 0.0431 & N/A & 1.0000 & 0.0021 \\
\hline LCT-009-017 & $1.30 \mathrm{E}-07$ & $3.88 \mathrm{E}+00$ & 0.0431 & N/A & 1.0000 & 0.0021 \\
\hline
\end{tabular}




\begin{tabular}{|c|c|c|c|c|c|c|}
\hline Case Name & EALF & $V_{m} / V_{f}$ & ${ }_{\overline{235} \mathbf{U} / \mathbf{U}}$ & $\mathbf{H} /{ }^{235} \mathrm{U}$ & $\mathbf{k}_{\text {bench }}$ & $\sigma_{\text {bench }}$ \\
\hline LCT-009-018 & $1.31 \mathrm{E}-07$ & $3.88 E+00$ & 0.0431 & $\mathrm{~N} / \mathrm{A}$ & 1.0000 & 0.0021 \\
\hline LCT-009-019 & $1.30 \mathrm{E}-07$ & $3.88 \mathrm{E}+00$ & 0.0431 & N/A & 1.0000 & 0.0021 \\
\hline LCT-009-020 & $1.31 \mathrm{E}-07$ & $3.88 \mathrm{E}+00$ & 0.0431 & $\mathrm{~N} / \mathrm{A}$ & 1.0000 & 0.0021 \\
\hline LCT-009-021 & $1.30 \mathrm{E}-07$ & $3.88 \mathrm{E}+00$ & 0.0431 & N/A & 1.0000 & 0.0021 \\
\hline LCT-009-022 & $1.31 \mathrm{E}-07$ & $3.88 \mathrm{E}+00$ & 0.0431 & N/A & 1.0000 & 0.0021 \\
\hline LCT-009-023 & $1.30 \mathrm{E}-07$ & $3.88 \mathrm{E}+00$ & 0.0431 & N/A & 1.0000 & 0.0021 \\
\hline LCT-009-024 & $1.29 \mathrm{E}-07$ & $3.88 \mathrm{E}+00$ & 0.0431 & N/A & 1.0000 & 0.0021 \\
\hline LCT-009-025 & $1.29 \mathrm{E}-07$ & $3.88 \mathrm{E}+00$ & 0.0431 & $\mathrm{~N} / \mathrm{A}$ & 1.0000 & 0.0021 \\
\hline LCT-009-026 & $1.29 \mathrm{E}-07$ & $3.88 \mathrm{E}+00$ & 0.0431 & N/A & 1.0000 & 0.0021 \\
\hline LCT-009-027 & $1.29 \mathrm{E}-07$ & $3.88 \mathrm{E}+00$ & 0.0431 & $\mathrm{~N} / \mathrm{A}$ & 1.0000 & 0.0021 \\
\hline LCT-010-001 & $1.36 \mathrm{E}-07$ & $3.88 \mathrm{E}+00$ & 0.0431 & $\mathrm{~N} / \mathrm{A}$ & 1.0000 & 0.0021 \\
\hline LCT-010-002 & $1.30 \mathrm{E}-07$ & $3.88 \mathrm{E}+00$ & 0.0431 & N/A & 1.0000 & 0.0021 \\
\hline LCT-010-003 & $1.28 \mathrm{E}-07$ & $3.88 \mathrm{E}+00$ & 0.0431 & $\mathrm{~N} / \mathrm{A}$ & 1.0000 & 0.0021 \\
\hline LCT-010-004 & $1.26 \mathrm{E}-07$ & $3.88 \mathrm{E}+00$ & 0.0431 & $\mathrm{~N} / \mathrm{A}$ & 1.0000 & 0.0021 \\
\hline LCT $-010-005$ & $4.87 \mathrm{E}-07$ & $3.88 \mathrm{E}+00$ & 0.0431 & N/A & 1.0000 & 0.0021 \\
\hline LCT-010-006 & $3.49 \mathrm{E}-07$ & $3.88 \mathrm{E}+00$ & 0.0431 & N/A & 1.0000 & 0.0021 \\
\hline LCT-010-007 & $2.68 \mathrm{E}-07$ & $3.88 \mathrm{E}+00$ & 0.0431 & N/A & 1.0000 & 0.0021 \\
\hline LCT-010-008 & $2.32 \mathrm{E}-07$ & $3.88 \mathrm{E}+00$ & 0.0431 & N/A & 1.0000 & 0.0021 \\
\hline LCT-010-009 & $1.41 \mathrm{E}-07$ & $3.88 \mathrm{E}+00$ & 0.0431 & N/A & 1.0000 & 0.0021 \\
\hline LCT $-010-010$ & $1.35 \mathrm{E}-07$ & $3.88 \mathrm{E}+00$ & 0.0431 & N/A & 1.0000 & 0.0021 \\
\hline LCT-010-011 & $1.33 \mathrm{E}-07$ & $3.88 \mathrm{E}+00$ & 0.0431 & N/A & 1.0000 & 0.0021 \\
\hline LCT-010-012 & $1.30 \mathrm{E}-07$ & $3.88 \mathrm{E}+00$ & 0.0431 & $\mathrm{~N} / \mathrm{A}$ & 1.0000 & 0.0021 \\
\hline LCT $-010-013$ & $1.27 \mathrm{E}-07$ & $3.88 \mathrm{E}+00$ & 0.0431 & N/A & 1.0000 & 0.0021 \\
\hline LCT-010-014 & $3.76 \mathrm{E}-07$ & $1.60 \mathrm{E}+00$ & 0.0431 & N/A & 1.0000 & 0.0028 \\
\hline LCT-010-015 & $3.61 \mathrm{E}-07$ & $1.60 \mathrm{E}+00$ & 0.0431 & $\mathrm{~N} / \mathrm{A}$ & 1.0000 & 0.0028 \\
\hline LCT-010-016 & $3.51 \mathrm{E}-07$ & $1.60 \mathrm{E}+00$ & 0.0431 & N/A & 1.0000 & 0.0028 \\
\hline LCT-010-017 & $3.41 \mathrm{E}-07$ & $1.60 \mathrm{E}+00$ & 0.0431 & N/A & 1.0000 & 0.0028 \\
\hline LCT-010-018 & $3.35 \mathrm{E}-07$ & $1.60 \mathrm{E}+00$ & 0.0431 & $\mathrm{~N} / \mathrm{A}$ & 1.0000 & 0.0028 \\
\hline LCT-010-019 & $3.27 \mathrm{E}-07$ & $1.60 \mathrm{E}+00$ & 0.0431 & N/A & 1.0000 & 0.0028 \\
\hline LCT $-010-020$ & $3.60 \mathrm{E}-07$ & $1.60 \mathrm{E}+00$ & 0.0431 & N/A & 1.0000 & 0.0028 \\
\hline LCT-010-021 & $3.49 \mathrm{E}-07$ & $1.60 \mathrm{E}+00$ & 0.0431 & N/A & 1.0000 & 0.0028 \\
\hline LCT-010-022 & $3.35 \mathrm{E}-07$ & $1.60 \mathrm{E}+00$ & 0.0431 & N/A & 1.0000 & 0.0028 \\
\hline LCT-010-023 & $3.26 \mathrm{E}-07$ & $1.60 \mathrm{E}+00$ & 0.0431 & N/A & 1.0000 & 0.0028 \\
\hline LCT-010-024 & $8.21 \mathrm{E}-07$ & $1.60 \mathrm{E}+00$ & 0.0431 & N/A & 1.0000 & 0.0028 \\
\hline LCT-010-025 & $7.47 \mathrm{E}-07$ & $1.60 \mathrm{E}+00$ & 0.0431 & N/A & 1.0000 & 0.0028 \\
\hline LCT-010-026 & $6.88 \mathrm{E}-07$ & $1.60 \mathrm{E}+00$ & 0.0431 & $\mathrm{~N} / \mathrm{A}$ & 1.0000 & 0.0028 \\
\hline $\begin{array}{l}\text { LCT-010-027 } \\
\end{array}$ & $6.41 \mathrm{E}-07$ & $1.60 \mathrm{E}+00$ & 0.0431 & N/A & 1.0000 & 0.0028 \\
\hline LCT-010-028 & $5.96 \mathrm{E}-07$ & $1.60 \mathrm{E}+00$ & 0.0431 & N/A & 1.0000 & 0.0028 \\
\hline LCT-010-029 & $5.57 \mathrm{E}-07$ & $1.60 \mathrm{E}+00$ & 0.0431 & $\mathrm{~N} / \mathrm{A}$ & 1.0000 & 0.0028 \\
\hline LCT-010-030 & $4.75 \mathrm{E}-07$ & $1.60 \mathrm{E}+00$ & 0.0431 & N/A & 1.0000 & 0.0028 \\
\hline LCT-026-001 & $2.80 \mathrm{E}-07$ & $2.15 \mathrm{E}+00$ & 0.0492 & N/A & 1.0000 & 0.0034 \\
\hline LCT-026-002 & $4.74 \mathrm{E}-07$ & $2.15 \mathrm{E}+00$ & 0.0492 & N/A & 0.9996 & 0.0034 \\
\hline LCT-026-003 & $1.20 \mathrm{E}-06$ & $1.22 \mathrm{E}+00$ & 0.0492 & N/A & 1.0018 & 0.0062 \\
\hline LCT-026-004 & $1.96 \mathrm{E}-06$ & $1.22 \mathrm{E}+00$ & 0.0492 & N/A & 0.9978 & 0.0062 \\
\hline LCT-026-005 & $4.53 \mathrm{E}-07$ & 8.97E-01 & 0.0492 & N/A & 1.0007 & 0.0041 \\
\hline LCT-026-006 & $6.62 \mathrm{E}-07$ & 8.97E-01 & 0.0492 & N/A & 0.9983 & 0.0041 \\
\hline LCT-042-001 & $2.06 \mathrm{E}-07$ & $1.60 \mathrm{E}+00$ & 0.0235 & N/A & 1.0000 & 0.0016 \\
\hline LCT-042-002 & $2.14 \mathrm{E}-07$ & $1.60 \mathrm{E}+00$ & 0.0235 & N/A & 1.0000 & 0.0016 \\
\hline LCT-042-003 & $2.23 \mathrm{E}-07$ & $1.60 \mathrm{E}+00$ & 0.0235 & $\mathrm{~N} / \mathrm{A}$ & 1.0000 & 0.0016 \\
\hline LCT-042-004 & $2.21 \mathrm{E}-07$ & $1.60 \mathrm{E}+00$ & 0.0235 & N/A & 1.0000 & 0.0017 \\
\hline
\end{tabular}


CHPRC-00455, REV. 0

\begin{tabular}{|c|c|c|c|c|c|c|}
\hline Case Name & EALF & $V_{m} / V_{f}$ & ${ }^{\overline{235} \mathrm{U} / \mathrm{U}}$ & $\bar{H} /{ }^{235} \mathrm{U}$ & $\mathbf{k}_{\text {bench }}$ & $\sigma_{\text {bench }}$ \\
\hline$\overline{\text { LCT-042-005 }}$ & $2.17 \mathrm{E}-07$ & $1.60 \mathrm{E}+00$ & 0.0235 & N/A & 1.0000 & 0.0033 \\
\hline LCT-042-006 & $2.06 \mathrm{E}-07$ & $1.60 \mathrm{E}+00$ & 0.0235 & $\mathrm{~N} / \mathrm{A}$ & 1.0000 & 0.0016 \\
\hline LCT-042-007 & $2.11 \mathrm{E}-07$ & $1.60 \mathrm{E}+00$ & 0.0235 & $\mathrm{~N} / \mathrm{A}$ & 1.0000 & 0.0018 \\
\hline LCT-049-001 & $2.49 \mathrm{E}-06$ & $2.01 \mathrm{E}+00$ & 0.0500 & $3.99 \mathrm{E}+01$ & 1.0000 & 0.0034 \\
\hline LCT-049-002 & $2.51 \mathrm{E}-06$ & $2.01 \mathrm{E}+00$ & 0.0500 & $3.99 \mathrm{E}+01$ & 1.0000 & 0.0034 \\
\hline LCT-049-003 & $2.67 \mathrm{E}-06$ & $2.01 \mathrm{E}+00$ & 0.0500 & $3.99 \mathrm{E}+01$ & 1.0000 & 0.0034 \\
\hline LCT-049-004 & $2.78 \mathrm{E}-06$ & $2.01 \mathrm{E}+00$ & 0.0500 & $3.99 \mathrm{E}+01$ & 1.0000 & 0.0034 \\
\hline LCT-049-005 & $1.40 \mathrm{E}-06$ & $2.52 \mathrm{E}+00$ & 0.0500 & $4.99 \mathrm{E}+01$ & 1.0000 & 0.0042 \\
\hline LCT-049-006 & $1.42 \mathrm{E}-06$ & $2.52 \mathrm{E}+00$ & 0.0500 & $4.99 \mathrm{E}+01$ & 1.0000 & 0.0042 \\
\hline LCT-049-007 & $1.36 \mathrm{E}-06$ & $2.52 \mathrm{E}+00$ & 0.0500 & $4.99 \mathrm{E}+01$ & 1.0000 & 0.0042 \\
\hline LCT-049-008 & $1.46 \mathrm{E}-06$ & $2.52 \mathrm{E}+00$ & 0.0500 & $4.99 \mathrm{E}+01$ & 1.0000 & 0.0042 \\
\hline LCT-049-009 & $8.99 \mathrm{E}-07$ & $3.01 \mathrm{E}+00$ & 0.0500 & $5.97 \mathrm{E}+01$ & 1.0000 & 0.0037 \\
\hline LCT $-049-010$ & $9.05 \mathrm{E}-07$ & $3.01 \mathrm{E}+00$ & 0.0500 & $5.97 \mathrm{E}+01$ & 1.0000 & 0.0037 \\
\hline LCT-049-011 & $9.02 \mathrm{E}-07$ & $3.01 \mathrm{E}+00$ & 0.0500 & $5.97 \mathrm{E}+01$ & 1.0000 & 0.0037 \\
\hline LCT-049-012 & $9.54 \mathrm{E}-07$ & $3.01 \mathrm{E}+00$ & 0.0500 & $5.97 \mathrm{E}+01$ & 1.0000 & 0.0037 \\
\hline LCT-049-013 & $1.73 \mathrm{E}-06$ & $3.01 \mathrm{E}+00$ & 0.0500 & $5.97 \mathrm{E}+01$ & 1.0000 & 0.0036 \\
\hline LCT-049-014 & $1.74 \mathrm{E}-06$ & $3.01 \mathrm{E}+00$ & 0.0500 & $5.97 \mathrm{E}+01$ & 1.0000 & 0.0036 \\
\hline LCT-049-015 & $1.74 \mathrm{E}-06$ & $3.01 \mathrm{E}+00$ & 0.0500 & $5.97 \mathrm{E}+01$ & 1.0000 & 0.0036 \\
\hline LCT-049-016 & $1.35 \mathrm{E}-06$ & $3.01 \mathrm{E}+00$ & 0.0500 & $5.97 \mathrm{E}+01$ & 1.0000 & 0.0036 \\
\hline LCT-049-017 & $1.45 \mathrm{E}-06$ & $3.01 \mathrm{E}+00$ & 0.0500 & $5.97 \mathrm{E}+01$ & 1.0000 & 0.0036 \\
\hline LCT-049-018 & $1.24 \mathrm{E}-06$ & $3.01 \mathrm{E}+00$ & 0.0500 & $5.97 \mathrm{E}+01$ & 1.0000 & 0.0030 \\
\hline LCT-052-001 & $5.23 \mathrm{E}-07$ & $2.14 \mathrm{E}+00$ & 0.0474 & $\mathrm{~N} / \mathrm{A}$ & 1.0003 & 0.0023 \\
\hline LCT-052-002 & $1.90 \mathrm{E}-07$ & $4.15 \mathrm{E}+00$ & 0.0474 & N/A & 1.0003 & 0.0036 \\
\hline LCT-052-003 & $9.46 \mathrm{E}-08$ & $7.95 \mathrm{E}+00$ & 0.0474 & $\mathrm{~N} / \mathrm{A}$ & 1.0003 & 0.0034 \\
\hline LCT-052-004 & $5.25 \mathrm{E}-07$ & $2.14 \mathrm{E}+00$ & 0.0474 & $\mathrm{~N} / \mathrm{A}$ & 1.0003 & 0.0023 \\
\hline LCT-052-005 & $1.91 \mathrm{E}-07$ & $4.15 \mathrm{E}+00$ & 0.0474 & $\mathrm{~N} / \mathrm{A}$ & 1.0003 & 0.0036 \\
\hline LCT-052-006 & $9.59 \mathrm{E}-08$ & $7.95 \mathrm{E}+00$ & 0.0474 & N/A & 1.0003 & 0.0034 \\
\hline LST-003-001 & $4.14 \mathrm{E}-08$ & $7.85 \mathrm{E}+01$ & 0.1000 & $7.70 \mathrm{E}+02$ & 0.9997 & 0.0039 \\
\hline LST-003-002 & $3.95 \mathrm{E}-08$ & $8.94 \mathrm{E}+01$ & 0.1000 & $8.78 \mathrm{E}+02$ & 0.9993 & 0.0042 \\
\hline LST-003-003 & $3.92 \mathrm{E}-08$ & $9.14 \mathrm{E}+01$ & 0.1000 & $8.97 \mathrm{E}+02$ & 0.9995 & 0.0042 \\
\hline LST-003-004 & $3.90 \mathrm{E}-08$ & $9.31 \mathrm{E}+01$ & 0.1000 & $9.13 \mathrm{E}+02$ & 0.9995 & 0.0042 \\
\hline LST-003-005 & $3.63 \mathrm{E}-08$ & $1.20 \mathrm{E}+02$ & 0.1000 & $1.17 \mathrm{E}+03$ & 0.9997 & 0.0048 \\
\hline LST-003-006 & $3.60 \mathrm{E}-08$ & $1.24 \mathrm{E}+02$ & 0.1000 & $1.21 \mathrm{E}+03$ & 0.9999 & 0.0049 \\
\hline LST-003-007 & $3.58 \mathrm{E}-08$ & $1.26 \mathrm{E}+02$ & 0.1000 & $1.24 \mathrm{E}+03$ & 0.9994 & 0.0049 \\
\hline LST-003-008 & $3.47 \mathrm{E}-08$ & $1.44 \mathrm{E}+02$ & 0.1000 & $1.41 \mathrm{E}+03$ & 0.9993 & 0.0052 \\
\hline LST-003-009 & $3.46 \mathrm{E}-08$ & $1.46 \mathrm{E}+02$ & 0.1000 & $1.44 \mathrm{E}+03$ & 0.9996 & 0.0052 \\
\hline LST-004-001 & $4.21 \mathrm{E}-08$ & $0.00 \mathrm{E}+00$ & 0.1000 & $7.19 \mathrm{E}+02$ & 0.9994 & 0.0008 \\
\hline LST-004-029 & $4.10 \mathrm{E}-08$ & $0.00 \mathrm{E}+00$ & 0.1000 & $7.71 \mathrm{E}+02$ & 0.9999 & 0.0009 \\
\hline LST-004-033 & $3.98 \mathrm{E}-08$ & $0.00 \mathrm{E}+00$ & 0.1000 & $8.42 \mathrm{E}+02$ & 0.9999 & 0.0009 \\
\hline LST-004-034 & $3.90 \mathrm{E}-08$ & $0.00 \mathrm{E}+00$ & 0.1000 & $8.96 \mathrm{E}+02$ & 0.9999 & 0.0010 \\
\hline LST-004-046 & $3.84 \mathrm{E}-08$ & $0.00 \mathrm{E}+00$ & 0.1000 & $9.42 \mathrm{E}+02$ & 0.9999 & 0.0010 \\
\hline LST-004-051 & $3.79 \mathrm{E}-08$ & $0.00 \mathrm{E}+00$ & 0.1000 & $9.83 \mathrm{E}+02$ & 0.9994 & 0.0011 \\
\hline LST-004-054 & $3.75 \mathrm{E}-08$ & $0.00 \mathrm{E}+00$ & 0.1000 & $1.02 \mathrm{E}+03$ & 0.9996 & 0.0011 \\
\hline LST-005-001 & $4.12 \mathrm{E}-08$ & $5.55 \mathrm{E}+01$ & 0.0564 & $9.72 \mathrm{E}+02$ & 1.0000 & 0.0042 \\
\hline LST-005-002 & $4.12 \mathrm{E}-08$ & $5.55 \mathrm{E}+01$ & 0.0564 & $9.72 \mathrm{E}+02$ & 1.0000 & 0.0051 \\
\hline LST-005-003 & $4.15 \mathrm{E}-08$ & $5.55 \mathrm{E}+01$ & 0.0564 & $9.72 \mathrm{E}+02$ & 1.0000 & 0.0064 \\
\hline LST-006-001 & $4.86 \mathrm{E}-08$ & $5.42 \mathrm{E}+01$ & 0.1000 & $5.32 \mathrm{E}+02$ & 1.0000 & 0.0037 \\
\hline LST-006-002 & $4.89 \mathrm{E}-08$ & $5.42 \mathrm{E}+01$ & 0.1000 & $5.32 \mathrm{E}+02$ & 1.0000 & 0.0038 \\
\hline LST-006-003 & $4.93 \mathrm{E}-08$ & $5.42 \mathrm{E}+01$ & 0.1000 & $5.32 \mathrm{E}+02$ & 1.0000 & 0.0041 \\
\hline LST-006-004 & $4.94 \mathrm{E}-08$ & $5.42 \mathrm{E}+01$ & 0.1000 & $5.32 \mathrm{E}+02$ & 1.0000 & 0.0041 \\
\hline
\end{tabular}




\section{CHPRC-00455, REV. 0}

\begin{tabular}{|l|r|r|r|r|r|r||}
\hline \multicolumn{1}{|c|}{ Case Name } & \multicolumn{1}{|c|}{$\mathbf{E A L F}$} & \multicolumn{1}{c|}{$\mathbf{V}_{\mathbf{m}} / \mathbf{V}_{\mathbf{f}}$} & ${ }^{235} \mathbf{U} / \mathbf{U}$ & \multicolumn{1}{c|}{$\mathbf{H}^{235} \mathbf{U}$} & \multicolumn{1}{c|}{$\mathbf{k}_{\text {bench }}$} & \multicolumn{1}{c|}{$\boldsymbol{\sigma}_{\text {bench }}$} \\
\hline LST-006-005 & $4.99 \mathrm{E}-08$ & $5.42 \mathrm{E}+01$ & 0.1000 & $5.32 \mathrm{E}+02$ & 1.0000 & 0.0047 \\
\hline LST-007-014 & $4.28 \mathrm{E}-08$ & $7.15 \mathrm{E}+01$ & 0.1000 & $7.09 \mathrm{E}+02$ & 0.9961 & 0.0009 \\
\hline LST-007-030 & $4.15 \mathrm{E}-08$ & $7.76 \mathrm{E}+01$ & 0.1000 & $7.70 \mathrm{E}+02$ & 0.9973 & 0.0009 \\
\hline LST-007-032 & $4.01 \mathrm{E}-08$ & $8.49 \mathrm{E}+01$ & 0.1000 & $8.42 \mathrm{E}+02$ & 0.9985 & 0.0010 \\
\hline LST-007-036 & $3.93 \mathrm{E}-08$ & $9.04 \mathrm{E}+01$ & 0.1000 & $8.96 \mathrm{E}+02$ & 0.9988 & 0.0011 \\
\hline LST-007-049 & $3.87 \mathrm{E}-08$ & $9.50 \mathrm{E}+01$ & 0.1000 & $9.42 \mathrm{E}+02$ & 0.9983 & 0.0011 \\
\hline LST-016-105 & $5.22 \mathrm{E}-08$ & $4.73 \mathrm{E}+01$ & 0.1000 & $4.69 \mathrm{E}+02$ & 0.9996 & 0.0013 \\
\hline LST-016-113 & $4.96 \mathrm{E}-08$ & $5.18 \mathrm{E}+01$ & 0.1000 & $5.14 \mathrm{E}+02$ & 0.9999 & 0.0013 \\
\hline LST-016-125 & $4.57 \mathrm{E}-08$ & $6.14 \mathrm{E}+01$ & 0.1000 & $6.08 \mathrm{E}+02$ & 0.9994 & 0.0014 \\
\hline LST-016-129 & $4.44 \mathrm{E}-08$ & $6.56 \mathrm{E}+01$ & 0.1000 & $6.50 \mathrm{E}+02$ & 0.9996 & 0.0014 \\
\hline LST-016-131 & $4.31 \mathrm{E}-08$ & $7.05 \mathrm{E}+01$ & 0.1000 & $6.99 \mathrm{E}+02$ & 0.9995 & 0.0014 \\
\hline LST-016-140 & $4.22 \mathrm{E}-08$ & $7.45 \mathrm{E}+01$ & 0.1000 & $7.39 \mathrm{E}+02$ & 0.9992 & 0.0015 \\
\hline LST-016-196 & $4.15 \mathrm{E}-08$ & $7.78 \mathrm{E}+01$ & 0.1000 & $7.72 \mathrm{E}+02$ & 0.9994 & 0.0015 \\
\hline LST-017-104 & $5.23 \mathrm{E}-08$ & $4.73 \mathrm{E}+01$ & 0.1000 & $4.69 \mathrm{E}+02$ & 0.9981 & 0.0013 \\
\hline LST-017-122 & $4.99 \mathrm{E}-08$ & $5.15 \mathrm{E}+01$ & 0.1000 & $5.11 \mathrm{E}+02$ & 0.9986 & 0.0013 \\
\hline LST-017-123 & $4.57 \mathrm{E}-08$ & $6.16 \mathrm{E}+01$ & 0.1000 & $6.11 \mathrm{E}+02$ & 0.9989 & 0.0014 \\
\hline LST-017-126 & $4.45 \mathrm{E}-08$ & $6.56 \mathrm{E}+01$ & 0.1000 & $6.50 \mathrm{E}+02$ & 0.9992 & 0.0014 \\
\hline LST-017-130 & $4.31 \mathrm{E}-08$ & $7.05 \mathrm{E}+01$ & 0.1000 & $6.99 \mathrm{E}+02$ & 0.9987 & 0.0015 \\
\hline LST-017-147 & $4.24 \mathrm{E}-08$ & $7.35 \mathrm{E}+01$ & 0.1000 & $7.29 \mathrm{E}+02$ & 0.9996 & 0.0015 \\
\hline
\end{tabular}

\subsection{CALCULATED MCNP RESULTS}

Table 2-3 below contains a listing of all of the validation MCNP results. The table lists the case name, the reported benchmark $\mathrm{k}_{\mathrm{eff}}$ and one-sigma $(1 \sigma)$ statistical error (from Table 2-2), the calculated MCNP $\mathrm{k}_{\text {eff }}$ and $1 \sigma$ statistical error, and the normalized $\mathrm{k}_{\text {eff }}$ and total statistical error. The $\mathrm{k}_{\mathrm{eff}}$ normalization ( $\mathrm{k}_{\text {norm }}$ ) was performed by dividing the calculated $\mathrm{k}_{\mathrm{eff}}\left(\mathrm{k}_{\mathrm{MCNP}}\right)$ by the reported benchmark $\mathrm{k}_{\mathrm{eff}}\left(\mathrm{k}_{\text {bench }}\right)$. The statistical error or the uncertainty value $\left(\sigma_{\text {total }}\right)$ assigned to each case is a combination of the benchmark uncertainty for each experiment $\sigma_{\text {bench }}$ and the Monte Carlo uncertainty associated with the particular computational evaluation of the case, $\sigma_{\mathrm{MCNP}}$, or:

$$
\left(\sigma_{\text {bench }}^{2}+\sigma_{\mathrm{MCNP}}{ }^{2}\right)^{1 / 2}
$$

Table 2-3. Case Name and MCNP Results

\begin{tabular}{|l|c|c|c|c|c|c|c|c|c|}
\hline Case Name & & $\mathbf{k}_{\text {bench }}$ & $\boldsymbol{\sigma}_{\text {bench }}$ & & $\mathbf{k}_{\text {MCNP }}$ & $\boldsymbol{\sigma}_{\text {MCNP }}$ & & $\mathbf{k}_{\text {norm }}$ & $\boldsymbol{\sigma}_{\text {total }}$ \\
\hline ICT-002-001 & & 1.0014 & 0.0039 & & 0.9936 & 0.0007 & & 0.9922 & 0.0040 \\
\hline ICT-002-002 & & 1.0019 & 0.0040 & & 0.9975 & 0.0008 & & 0.9956 & 0.0041 \\
\hline ICT-002-003 & 1.0017 & 0.0044 & & 0.9986 & 0.0007 & & 0.9969 & 0.0045 \\
\hline ICT-002-004 & 1.0019 & 0.0044 & & 0.9933 & 0.0008 & & 0.9914 & 0.0045 \\
\hline ICT-002-005 & 1.0014 & 0.0043 & & 0.9931 & 0.0007 & & 0.9917 & 0.0044 \\
\hline ICT-002-006 & 1.0016 & 0.0044 & & 0.9875 & 0.0007 & & 0.9859 & 0.0045 \\
\hline LCT-002-001 & 0.9997 & 0.0020 & & 0.9929 & 0.0020 & & 0.9932 & 0.0028 \\
\hline LCT-002-002 & 0.9997 & 0.0020 & & 0.9947 & 0.0019 & & 0.9950 & 0.0028 \\
\hline LCT-002-003 & 0.9997 & 0.0020 & & 0.9994 & 0.0019 & & 0.9997 & 0.0028 \\
\hline LCT-002-004 & 0.9997 & 0.0020 & & 0.9983 & 0.0020 & & 0.9986 & 0.0028 \\
\hline LCT-002-005 & 0.9997 & 0.0020 & & 0.9942 & 0.0019 & & 0.9945 & 0.0028 \\
\hline
\end{tabular}


CHPRC-00455, REV. 0

\begin{tabular}{|c|c|c|c|c|c|c|}
\hline Case Name & $\mathbf{k}_{\text {bench }}$ & $\sigma_{\text {bench }}$ & $\mathbf{k}_{\mathrm{MCNP}}$ & $\sigma_{\mathrm{MCNP}}$ & $\mathbf{k}_{\text {norm }}$ & $\sigma_{\text {total }}$ \\
\hline LCT-004-001 & 0.9998 & 0.0033 & 0.9923 & 0.0018 & 0.9925 & 0.0038 \\
\hline LCT-004-002 & 9998 & 0.0033 & .9890 & 0.0022 & .9892 & 0.0040 \\
\hline LCT-004-003 & 9998 & 0.0033 & 0.9935 & 0.0021 & .9937 & 0.0039 \\
\hline LCT-004-004 & 0.9998 & 0.0033 & 0.9898 & 0.0019 & 0.9900 & 0.0038 \\
\hline LCT-004-005 & 0.9998 & 0.0033 & 0.9921 & 0.0019 & 0.9923 & 0.0038 \\
\hline LCT-004-006 & 0.9998 & 0.0033 & 0.9870 & 0.0023 & 0.9872 & 0.0040 \\
\hline LCT-004-007 & .9998 & 0.0033 & 0.9891 & 0.0021 & 0.9893 & 0.0039 \\
\hline LCT-004-008 & 9998 & 0.0035 & 0.9852 & 0.0021 & .9853 & 0.0041 \\
\hline LCT-004-009 & 9998 & 0.0035 & 0.9853 & 0.0018 & .9855 & 0.0040 \\
\hline LCT-004-010 & 0.9998 & 0.0035 & 0.9969 & 0.0019 & .9971 & 0040 \\
\hline LCT-004-011 & 0.9998 & 0.0035 & 0.9828 & 0.0020 & 0.9830 & 0.0040 \\
\hline LCT-004-012 & 0.9998 & 0.0035 & 0.9745 & 0.0017 & 0.9746 & 0.0039 \\
\hline LCT-004-013 & 0.9998 & 0.0035 & 0.9818 & 0.0020 & 0.9820 & 0.0040 \\
\hline LCT-C & 9998 & 0.0 & 0.9879 & 0.0023 & 0.9881 & 0.0042 \\
\hline & 9998 & & & & 825 & \\
\hline LCT & 0.9998 & 0.0 & 0.9867 & 0.0 & .9869 & 0040 \\
\hline LCT-004-017 & 0.9998 & 0.0035 & 0.9854 & 0.0020 & 0.9856 & 0.0040 \\
\hline LCT-004-018 & 0.9998 & 0.0035 & 0.9818 & 0.0019 & 9820 & 0.0040 \\
\hline LCT-004-019 & 0.9998 & 0.0 & 0.9886 & 0.0019 & 0.9888 & 0.0040 \\
\hline LCT & 9998 & 0.0 & 9838 & 0.0017 & .9839 & 2039 \\
\hline LC1 & 0000 & 0.0 & 0.9936 & 0.0 & 0.9936 & 027 \\
\hline & 0000 & & & & 954 & \\
\hline LCT-0 & 1.0000 & 0.0 & 9957 & 0.0 & .9957 & 0.0027 \\
\hline LCT-009-004 & 1.0000 & 0.0 & 0.9950 & 0.0 & .9950 & 0.0029 \\
\hline LCT-009-005 & 1.0000 & 0.0 & 0.9966 & & .9966 & 0.0028 \\
\hline LCT-009-006 & 1.0000 & 0.0 & 0.9939 & 0.0 & 0.9939 & 0.0028 \\
\hline 007 & 0000 & 0.0 & 9964 & 0.0 & 0.9964 & 027 \\
\hline LC1 & 0000 & 0.0 & 0.9930 & & 0.9930 & \\
\hline LCT & 1.0000 & 0.0 & 0.9946 & & 0.9946 & 028 \\
\hline LCT-009-010 & 1.0000 & & 1.0006 & & \begin{tabular}{|l|}
1.0006 \\
\end{tabular} & 0.0029 \\
\hline LCT-0 & 1.0000 & & 0.9967 & & 0.9967 & 0.0027 \\
\hline LCT-009-012 & 1.0000 & 0.0 & 0.9966 & & 0.9966 & 0.0027 \\
\hline LCT-009-013 & 1.0000 & 0.0021 & \begin{tabular}{|l|}
1.0006 \\
\end{tabular} & & 1.0006 & 0.0027 \\
\hline & 1.0000 & 0.0021 & 0.9956 & 0.0 & 0.9956 & 0.0029 \\
\hline LCT-0 & 1.0000 & 0.0021 & 0.9923 & 0.0 & 0.9923 & 0.0027 \\
\hline LCT-009-016 & 1.0000 & 0.0021 & \begin{tabular}{|l|}
0.9932 \\
\end{tabular} & 0.0019 & \begin{tabular}{|l|}
0.9932 \\
\end{tabular} & 0.0028 \\
\hline & 1.0000 & & 1.0018 & 0.0020 & 1.0018 & 0.0029 \\
\hline LCT-009-018 & 1.0000 & 0.0021 & \begin{tabular}{|l|}
0.9958 \\
\end{tabular} & & 0.9958 & 0.0027 \\
\hline LCT-009-019 & 1.0000 & 0.0021 & 0.9931 & 0.0018 & 0.9931 & 0.0028 \\
\hline LCT-009-020 & 1.0000 & 0.0021 & 0.9968 & & 0.9968 & 0.0028 \\
\hline & 1.0000 & 0.0021 & 0.9954 & 0.0019 & 0.9954 & 0.0028 \\
\hline LCT-009-022 & 1.0000 & 0.0021 & 0.9994 & 0.0018 & 0.9994 & 0.0027 \\
\hline LCT-009-023 & 1.0000 & 0.0021 & \begin{tabular}{|l|}
0.9958 \\
\end{tabular} & & 0.9958 & 0.0028 \\
\hline LCT-009-024 & 1.0000 & 0.0021 & 0.9945 & 0.0018 & 0.9945 & 0.0028 \\
\hline LCT-009-025 & 1.0000 & 0.0021 & 0.9982 & 0.0017 & 0.9982 & 0.0027 \\
\hline LCT-009-026 & 1.0000 & 0.0021 & 0.9894 & 0.0017 & 0.9894 & 0.0027 \\
\hline LCT-009-027 & 1.0000 & & 0.9925 & 0.0019 & 0.9925 & 0.0028 \\
\hline LCT-010-001 & 1.0000 & 0.0021 & 1.0059 & 0.0019 & \begin{tabular}{|l|}
1.0059 \\
\end{tabular} & 0.0028 \\
\hline LCT-010-002 & 1.0000 & 0.0021 & 1.0075 & 0.0017 & 1.0075 & 0.0027 \\
\hline LCT-010-003 & 1.0000 & 0.0021 & 1.0017 & 0.0019 & 1.0017 & 0.0029 \\
\hline
\end{tabular}


CHPRC-00455, REV. 0

\begin{tabular}{|c|c|c|c|c|c|c|}
\hline Case Name & $\mathbf{k}_{\text {bench }}$ & $\sigma_{\text {bench }}$ & $\mathbf{k}_{\text {MCNP }}$ & $\sigma_{\mathrm{MCNP}}$ & $\mathbf{k}_{\text {norm }}$ & $\sigma_{\text {total }}$ \\
\hline LCT-010-004 & 1.0000 & 0.0021 & 0.9918 & 0.0017 & 0.9918 & 0.0027 \\
\hline LCT-010-005 & 1.0000 & 0.0021 & 0.9945 & 0.0019 & 0.9945 & 0.0029 \\
\hline LCT-010-006 & 1.0000 & 0.0021 & 0.9966 & 0.0018 & 0.9966 & 0.0028 \\
\hline LCT-010-007 & 1.0000 & 0.0021 & 0.9963 & 0.0017 & 0.9963 & 0.0027 \\
\hline LCT-010-008 & 1.0000 & 0.0021 & 0.9946 & 0.0019 & 0.9946 & 0.0029 \\
\hline LCT-010-009 & 1.0000 & 0.0021 & 0.9988 & 0.0018 & 0.9988 & 0.0027 \\
\hline LCT-010-010 & 1.0000 & 0.0021 & 0.9987 & 0.0015 & 0.9987 & 0.0026 \\
\hline LCT-010-011 & 1.0000 & 0.0021 & 0.9993 & 0.0019 & 0.9993 & 0.0028 \\
\hline LCT-010-012 & 1.0000 & 0.0021 & 0.9978 & 0.0019 & 0.9978 & 0.0028 \\
\hline LCT-010-013 & 1.0000 & 0.0021 & 0.9950 & 0.0018 & 0.9950 & 0.0028 \\
\hline LCT-010-014 & 1.0000 & 0.0028 & 0.9966 & 0.0019 & 0.9966 & 0.0034 \\
\hline LCT-010-015 & 1.0000 & 0.0028 & 0.9989 & 0.0017 & 0.9989 & 0.0033 \\
\hline LCT-010-016 & 1.0000 & 0.0028 & 1.0029 & 0.0018 & 1.0029 & 0.0033 \\
\hline LCT-010-017 & 1.0000 & 0.0028 & 0.9960 & 0.0018 & 0.9960 & 0.0033 \\
\hline LCT-010-018 & 1.0000 & 0.0028 & 0.9942 & 0.0020 & 0.9942 & 0.0034 \\
\hline LCT-010-019 & 1.0000 & 0.0028 & 0.9963 & 0.0021 & 0.9963 & 0.0035 \\
\hline LCT-010-020 & 1.0000 & 0.0028 & 1.0033 & 0.0019 & 1.0033 & 0.0034 \\
\hline LCT-010-021 & 1.0000 & 0.0028 & 1.0056 & 0.0018 & 1.0056 & 0.0033 \\
\hline LCT-010-022 & 1.0000 & 0.0028 & 1.0064 & 0.0018 & 1.0064 & 0.0033 \\
\hline LCT-010-023 & 1.0000 & 0.0028 & 0.9978 & 0.0018 & 0.9978 & 0.0034 \\
\hline LCT-010-024 & 1.0000 & 0.0028 & 0.9978 & 0.0017 & 0.9978 & 0.0033 \\
\hline LCT-010-025 & 1.0000 & 0.0028 & 0.9978 & 0.0017 & 0.9978 & 0.0033 \\
\hline LCT-010-026 & 1.0000 & 0.0028 & 1.0000 & 0.0017 & 1.0000 & 0.0033 \\
\hline LCT-010-027 & 1.0000 & 0.0028 & 1.0015 & 0.0017 & 1.0015 & 0.0033 \\
\hline LCT-010-028 & 1.0000 & 0.0028 & 0.9961 & 0.0019 & 0.9961 & 0.0034 \\
\hline LCT-010-029 & 1.0000 & 0.0028 & 0.9990 & 0.0019 & 0.9990 & 0.0034 \\
\hline LCT-010-030 & 1.0000 & 0.0028 & 0.9921 & 0.0018 & 0.9921 & 0.0033 \\
\hline LCT-026-001 & 1.0000 & 0.0034 & 0.9990 & 0.0008 & 0.9990 & 0.0035 \\
\hline LCT-026-002 & 0.9996 & 0.0034 & 1.0047 & 0.0008 & 1.0051 & 0.0035 \\
\hline LCT-026-003 & 1.0018 & 0.0062 & 1.0005 & 0.0008 & 0.9987 & 0.0062 \\
\hline LCT-026-004 & 0.9978 & 0.0062 & 1.0055 & 0.0008 & 1.0077 & 0.0062 \\
\hline LCT-026-005 & 1.0007 & 0.0041 & 0.9991 & 0.0008 & 0.9984 & 0.0042 \\
\hline LCT-026-006 & 0.9983 & 0.0041 & 1.0048 & 0.0008 & 1.0065 & 0.0042 \\
\hline LCT-042-001 & 1.0000 & 0.0016 & 0.9969 & 0.0016 & 0.9969 & 0.0023 \\
\hline LCT-042-002 & 1.0000 & 0.0016 & 0.9932 & 0.0016 & 0.9932 & 0.0023 \\
\hline LCT-042-003 & 1.0000 & 0.0016 & 0.9968 & 0.0016 & 0.9968 & 0.0022 \\
\hline LCT-042-004 & 1.0000 & 0.0017 & 0.9927 & 0.0017 & 0.9927 & 0.0024 \\
\hline LCT-042-005 & 1.0000 & 0.0033 & 0.9957 & 0.0018 & 0.9957 & 0.0037 \\
\hline LCT-042-006 & 1.0000 & 0.0016 & 0.9966 & 0.0017 & 0.9966 & 0.0023 \\
\hline LCT-042-007 & 1.0000 & 0.0018 & 0.9942 & 0.0017 & 0.9942 & 0.0024 \\
\hline LCT-049-001 & 1.0000 & 0.0034 & 0.9950 & 0.0006 & 0.9950 & 0.0035 \\
\hline LCT-049-002 & 1.0000 & \begin{tabular}{|l|}
0.0034 \\
\end{tabular} & 0.9947 & 0.0006 & 0.9947 & 0.0034 \\
\hline LCT-049-003 & 1.0000 & 0.0034 & 0.9947 & 0.0006 & 0.9947 & 0.0035 \\
\hline LCT-049-004 & 1.0000 & 0.0034 & 0.9948 & 0.0006 & 0.9948 & 0.0035 \\
\hline LCT-049-005 & 1.0000 & 0.0042 & 0.9957 & 0.0006 & 0.9957 & 0.0042 \\
\hline LCT-049-006 & 1.0000 & 0.0042 & 0.9961 & 0.0007 & 0.9961 & 0.0043 \\
\hline LCT-049-007 & 1.0000 & 0.0042 & 0.9953 & 0.0006 & 0.9953 & 0.0042 \\
\hline LCT-049-008 & 1.0000 & 0.0042 & 0.9930 & 0.0006 & 0.9930 & 0.0042 \\
\hline LCT-049-009 & 1.0000 & 0.0037 & 0.9948 & 0.0006 & 0.9948 & 0.0038 \\
\hline LCT-049-010 & 1.0000 & 0.0037 & 0.9970 & 0.0007 & 0.9970 & 0.0038 \\
\hline
\end{tabular}


CHPRC-00455, REV. 0

\begin{tabular}{|c|c|c|c|c|c|c|}
\hline Case Name & $\mathbf{k}_{\text {bench }}$ & $\sigma_{\text {bench }}$ & $\mathbf{k}_{\mathrm{MCNP}}$ & $\sigma_{M C N P}$ & $\mathbf{k}_{\text {norm }}$ & $\sigma_{\text {total }}$ \\
\hline LCT-049-011 & 1.0000 & 0.0037 & 0.9928 & 0.0007 & 0.9928 & 0.0038 \\
\hline LCT-049-012 & 1.0000 & 0.0037 & 0.9940 & 0.0007 & 0.9940 & 0.0038 \\
\hline LCT-049-013 & 1.0000 & 0.0036 & 0.9949 & 0.0006 & 0.9949 & 0.0037 \\
\hline LCT-049-014 & 1.0000 & 0.0036 & .9939 & 0.0007 & 0.9939 & 0.0037 \\
\hline LCT-049-015 & 1.0000 & 0.0036 & 9953 & 0.0007 & 0.9953 & 0.0037 \\
\hline LCT-049-016 & 1.0000 & 0.0036 & 0.9948 & 0.0006 & 0.9948 & 0.0037 \\
\hline LCT-049-017 & 1.0000 & 0.0036 & 0.9958 & 0.0006 & 0.9958 & 0.0036 \\
\hline LCT-049-018 & 1.0000 & 0.0030 & 0.9958 & 0.0006 & 0.9958 & 0.0031 \\
\hline LCT-052-001 & 1.0003 & 0.0023 & 0.9909 & 0.0006 & 0.9906 & 0.0024 \\
\hline LCT-052-002 & 1.0003 & 0.0036 & 0.9933 & 0.0005 & 0.9930 & 0.0036 \\
\hline LCT-052-003 & 1.0003 & & 0.9993 & 0.0005 & 0.9990 & \\
\hline LCT-052-004 & 1.0003 & 0.0023 & 0.9953 & 0.0005 & 0.9950 & 0.0024 \\
\hline LCT-052-005 & 1.0003 & 0.0036 & 0.9918 & 0.0005 & 0.9915 & 0.0036 \\
\hline LCT-0 & 1.0003 & 0.0034 & 0.9890 & 0.0005 & 0.9887 & 0.0034 \\
\hline LST-003-001 & 0.9997 & 0.0039 & 0.9949 & 0.0004 & 0.9952 & 0.0039 \\
\hline LST-003-002 & 0.9993 & 0.0042 & 0.9938 & 0.0004 & 0.9945 & 0.0042 \\
\hline LST-003-003 & 0.9995 & 0.0042 & 0.9980 & 0.0004 & 0.9985 & 0.0042 \\
\hline LST-003-004 & 9995 & 0.0042 & & 0.0004 & & \\
\hline LST-C & 0.9997 & & 9957 & 0.0003 & 0.9960 & 0.0 \\
\hline LS & .9999 & 0.0 & 9970 & 0.0003 & 0.9971 & 0.0049 \\
\hline LST-0 & .9994 & 0.0049 & 0.9946 & 0.0003 & 0.9952 & 0.0049 \\
\hline LST-003-008 & 0.9993 & 0.0052 & 0.9984 & 0.0003 & 0.9991 & 0.0052 \\
\hline LST-003-009 & 0.9996 & 0.0052 & 0.9967 & 0.0003 & 0.9971 & 0.0052 \\
\hline LST-004-001 & 0.9994 & 0.0008 & 0.9984 & 0.0007 & 0.9990 & 0.0010 \\
\hline LST-004-029 & 0.9999 & 0.0009 & 0.9983 & 0.0007 & 0.9984 & 0.0011 \\
\hline LST-0 & 9999 & & & 0.0006 & 0.9974 & \\
\hline LST & 0.9999 & & 0.9998 & 0.0006 & 0.9999 & \\
\hline LST & 0.9999 & 0.0 & 1.0001 & 0.0006 & 1.0002 & 0.0011 \\
\hline LST. & .9994 & 0.0011 & 0.9994 & 0.0006 & 0.9999 & 0.0012 \\
\hline LST-004-054 & 0.9996 & 0.0011 & 0.9991 & 0.0005 & 0.9995 & 0.0012 \\
\hline LST-005-001 & 1.0000 & 0.0042 & 0.9958 & 0.0005 & 0.9958 & 0.0042 \\
\hline LST-005-002 & 1.0000 & 0.0051 & 0.9965 & 0.0005 & 0.9965 & 0.0051 \\
\hline LST-005-003 & 1.0000 & 0.0064 & 0.9972 & 0.0005 & 0.9972 & 0.0064 \\
\hline LST-0 & 1.0000 & 0.0037 & 0.9986 & 0.0007 & 0.9986 & 0.0038 \\
\hline LST & 1.0000 & & & 0.0007 & 1.0038 & \\
\hline-003 & 1.0000 & 0.0041 & 0.9965 & 0.0008 & 0.9965 & 0.0042 \\
\hline LST-006-004 & 1.0000 & 0.0041 & 0.9987 & 0.0007 & 0.9987 & 0.0042 \\
\hline LST-006-005 & 1.0000 & 0.0047 & 0.9995 & 0.0007 & 0.9995 & 0.0048 \\
\hline LST-007-014 & 0.9961 & 0.0009 & 0.9927 & 0.0009 & 0.9966 & 0.0013 \\
\hline LST-007-030 & 0.9973 & 0.0009 & 0.9967 & 0.0008 & 0.9994 & 0.0012 \\
\hline LST-007-032 & 0.9985 & 0.0010 & 0.9953 & 0.0008 & 0.9968 & 0.0013 \\
\hline LST-007-036 & & & & & & \\
\hline LST-007-049 & & & & 0.0007 & 0.9974 & 0.0013 \\
\hline LST-016-105 & 0.9996 & 0.0013 & 1.0034 & 0.0009 & 1.0038 & 0.0016 \\
\hline LST-016-113 & 0.9999 & 0.0013 & 1.0048 & 0.0009 & 1.0049 & 0.0016 \\
\hline LST-016-125 & 0.9994 & 0.0014 & 1.0010 & 0.0008 & 1.0016 & 0.0016 \\
\hline LST-016-129 & 0.9996 & 0.0014 & 1.0020 & 0.0009 & 1.0024 & 0.0016 \\
\hline LST-016-131 & 0.9995 & 0.0014 & 0.9996 & 0.0008 & 1.0001 & 0.0016 \\
\hline LST-016-140 & 0.9992 & 0.0015 & 1.0014 & 0.0008 & 1.0022 & 0.0017 \\
\hline LST-016-196 & 0.9994 & 0.0015 & 1.0011 & 0.0008 & 1.0017 & 0.0017 \\
\hline
\end{tabular}


CHPRC-00455, REV. 0

\begin{tabular}{||c|c|c|c|c|c|c|c|c|c|}
\hline Case Name & & $\mathbf{k}_{\text {bench }}$ & $\boldsymbol{\sigma}_{\text {bench }}$ & & $\mathbf{k}_{\text {MCNP }}$ & $\boldsymbol{\sigma}_{\text {MCNP }}$ & & $\mathbf{k}_{\text {norm }}$ & $\boldsymbol{\sigma}_{\text {total }}$ \\
\hline LST-017-104 & & 0.9981 & 0.0013 & & 1.0016 & 0.0009 & & 1.0035 & 0.0016 \\
\hline LST-017-122 & & 0.9986 & 0.0013 & & 1.0013 & 0.0009 & & 1.0027 & 0.0016 \\
\hline LST-017-123 & & 0.9989 & 0.0014 & & 0.9992 & 0.0009 & & 1.0003 & 0.0017 \\
\hline LST-017-126 & & 0.9992 & 0.0014 & & 0.9991 & 0.0008 & & 0.9998 & 0.0016 \\
\hline LST-017-130 & & 0.9987 & 0.0015 & & 1.0005 & 0.0008 & & 1.0018 & 0.0017 \\
\hline LST-017-147 & & 0.9996 & 0.0015 & & 1.0001 & 0.0007 & & 1.0005 & 0.0017 \\
\hline
\end{tabular}

\subsection{STATISTICAL ANALYSIS}

The Oak Ridge National Laboratory (ORNL) code USLSTATS (ORNL 1998) was used to perform the statistical analysis of all case results. USLSTATS provides an automated means of evaluating and combining the statistical error of the calculation, code biases and benchmark uncertainties. The USLSTATS calculation uses the combined uncertainties and data to provide a linear trend and an overall uncertainty that would be inflated if simpler methods were used due to multicolinearity of the data.

The derived USL is determined such that there is a high degree of confidence that a calculated result is sub-critical if its calculated $k_{s}$ plus calculational uncertainties $\left(\Delta \mathrm{k}_{\mathrm{s}}\right)$ lies at or below this limit, i.e.,

$$
\mathrm{k}_{\mathrm{s}}+\Delta \mathrm{k}_{\mathrm{s}} \leq \mathrm{USL}
$$

Two different methods are used to determine of a USL for a set of criticality benchmark calculations. The USLSTATS computer program uses these two methods [i.e., (1) confidence band with administrative margin and (2) single-sided uniform-width closed-interval] to calculate USLs based on a set of user-supplied $\mathrm{k}_{\text {eff }}$ values and corresponding values of a single associated trending parameter $\mathrm{X}$ [e.g., EALF, $\mathrm{V}_{\mathrm{m}} / \mathrm{V}_{\mathrm{f}}$ ratio, ${ }^{235} \mathrm{U} / \mathrm{U}$ ratio, and $\mathrm{H} /{ }^{235} \mathrm{U}$ ratio], for a set of criticality benchmark calculations. USLSTATS performs a linear regression on the data to meet the confidence of fit requested.

Only USL method 1 (confidence band with administrative margin) results are utilized for this analysis. The recommended purpose of method 2 is to apply it in tandem with method 1 to verify that the administrative margin is conservative relative to a quantitative statistical estimate (see Figure 3-1). This application of method 2 may be especially important when a limited number of data points are used in the determination of benchmark $k_{\text {eff }}$ calculations or when the calculated values have a large standard deviation. In these cases the statistical-based subcritical margin may indicate the need for a larger administration margin in method 1.

USLSTATS uses several user input parameters to establish an appropriate USL. The user guide (ORNL 1998) has recommended values for each of these parameters:

- $\mathrm{P}$, proportion of population falling above the lower tolerance level $(\mathrm{P}=0.995)$. This parameter is used only in the computation of the USL by method 2, and represents the 
proportion of the population of criticality experiments that will lie above the line representing one tolerance level below the linear regression fit to $\mathrm{k}_{\text {eff. }}$

- $1-\gamma$, confidence of fit $(1-\gamma=0.95)$. This represents the desired confidence level to be assigned the linear regression fit of $k_{\text {eff }}$ values. It is also used in a $\chi^{2}$ test for normality of input data.

- $\alpha$, confidence on proportion $\mathrm{P}(\alpha=0.95)$. This parameter is a required input, but is not used by USL method 1

- $\Delta \mathrm{k}_{\mathrm{a}}$, administrative margin used to ensure subcriticality $\left(\Delta \mathrm{k}_{\mathrm{a}}=0.05\right)$. This parameter is only used by USL method 1 . This value is also consistent with the administrative margin normally used for criticality analyses with plutonium systems.

For this analysis each of the four parameters presented in Table 2-2 was used in conjunction with the normalized $\mathrm{k}_{\text {eff }}\left(\mathrm{k}_{\text {norm }}\right)$ and total uncertainty $\left(\sigma_{\text {total }}\right)$ from Table 2-3 to determine the appropriate USL. The results of the USLSTATS analysis are presented in Table 31 , which includes trending parameters, the USL equation for method 1, trending parameter range, and the minimum $\mathrm{k}_{\mathrm{eff}}$ values taken directly from the output files shown in Appendix D.

Table 3-1. USLSTATS Analysis Results

\begin{tabular}{|c|c|c|c|}
\hline $\begin{array}{l}\text { Trending } \\
\text { Parameter }\end{array}$ & USL Equation (Method 1) & $\begin{array}{c}\text { Trending Parameter } \\
\text { Range }\end{array}$ & $\begin{array}{c}\text { USL at } X=0 \\
\text { intercept }\end{array}$ \\
\hline EALF & $0.9353+(-4.4477 \mathrm{E}+02)^{*} \mathrm{X}$ & $3.46 \mathrm{E}-08<=\mathrm{X}<=2.78 \mathrm{E}-06$ & 0.934 \\
\hline $\mathrm{V}_{\mathrm{m}} / \mathrm{V}_{\mathrm{f}}$ & $\begin{array}{lr}0.9348+(3.7479 \mathrm{E}-05) * X(X<126.06) \\
0.9395 & (X>=126.06) \\
\end{array}$ & $0.0000<=X<=146.48$ & 0.935 \\
\hline${ }^{235} \mathrm{U} / \mathrm{U}$ & $0.9338+(2.7358 \mathrm{E}-02)^{*} \mathrm{X}$ & $2.35 \mathrm{E}-02<=\mathrm{X}<=0.17000$ & 0.934 \\
\hline $\mathrm{H} /(\mathrm{U}+\mathrm{Pu})$ & $0.9386+(2.5141 \mathrm{E}-06)^{*} \mathrm{X}$ & $39.943<=\mathrm{X}<=1437.5$ & 0.939 \\
\hline
\end{tabular}

The $\mathrm{k}_{\text {eff }}$ data, including the USLSTATS equation results as a function of each of the four trending parameters is also presented in Figure 3-1. In the figures, the data points are the $k_{\text {eff }}$ values. The upper line (long dashes) represents a linear regression fit to a set of calculations based on critical experiments $k_{c}(x)$. The middle line (doted) represents the lower confidence bend for a single additional calculation $\mathrm{k}_{\mathrm{c}}(\mathrm{x})-\mathrm{W}$. The width of this band $\mathrm{W}$ is determined statistically based on the existing data and a specific level of confidence, the greater the standard deviation in the data or the larger the confidence desired, the larger the bandwidth will be. The confidence band accounts for uncertainties in the experiments, the calculational approach, and the calculational data (e.g., cross sections). The value $\mathrm{W}$ is defined for a confidence level as

$$
\mathrm{W}=\max \left\{\left.\mathrm{W}(\mathrm{x})\right|_{\mathrm{x}-\min , \mathrm{x}-\max }\right\},
$$

where $\mathrm{w}(\mathrm{x})$ is a curvilinear function based on the pooled standard deviation of the linear regression fit and the standard deviation of each calculated $\mathrm{k}_{\mathrm{eff}}$ value. Note that the magnitude of $\mathrm{w}(\mathrm{x})$ is depended on the number of critical experiments included in the validation. Typically, $\mathrm{W}$ is determined at a $95 \%$ confidence level. 
In Figure 3-1 below, the USL for the EALF trending parameter curve is shown to trend downward slightly for higher EALFs with a few data points falling below the $\mathrm{k}_{\mathrm{c}}(\mathrm{x})$-W curve, the lower confidence limit for calculated criticals. For the 167 data points used, about 8 points could be below the curve for the $95 \%$ confidence level used. The bottom line of USL (1) in Fig. 3-1 represents the USL from method 1 , which has a quantity $\Delta \mathrm{k}_{\mathrm{a}}$ (defined earlier) as an additional margin below the $\mathrm{k}_{\mathrm{c}}(\mathrm{x})-\mathrm{W}$ line. The USL equation for the EALF gives a conservative upper subcritical limit of 0.934 when USL equation 1 in Table 3-1 is applied at the maximum trending parameter range.

The moderator to fissile volume ratio is used as the second trending parameter for the benchmark cases. The USL value is a minimum at the minimum parameter value. A positive bias adjustment line has been added to the line $\mathrm{k}_{\mathrm{c}}(\mathrm{x})-\mathrm{W}$ at the point where $\mathrm{k}_{\mathrm{c}}(\mathrm{x})=1$ to prevent taking credit for a positive bias $[\beta(x)>0]$ by assuming $k_{c}(x)=1$ everywhere that $k_{c}(x)>1$. The USL equation for the moderator to fissile volume ratio gives a conservative upper subcritical limit of 0.935 at the minimum parameter value.

The ratio of ${ }^{239} \mathrm{Pu}$ to the total heavy metal concentration is the third trending parameter for the benchmark cases. However, this trending parameter has been replaced by the uranium enrichments, which is shown to trend upward slightly for higher ${ }^{235} \mathrm{U} / \mathrm{U}$ ratios, but is essentially a horizontal line with a few data points falling below the $\mathrm{k}_{\mathrm{c}}(\mathrm{x})$-W curve, the lower confidence limit for calculated criticals. For the 167 data points used, about 8 points could be below the curve for the $95 \%$ confidence level used. Therefore, a conservative upper subcritical limit of 0.934 obtained from USL equation evaluated at $\mathrm{x}=0$ as shown in Table 3-1 would represent the lowest $\mathrm{k}_{\text {eff }}$ for this trending parameter from the 0.05 administrative margin line.

The ratio of the hydrogen concentration to the fissile concentration is used as the last trending parameter for the benchmark cases. Only the nitrate solution cases (60) as shown in Table 2-1 are included in the trending analysis while the heterogeneous cases are excluded even though moderator exists within the fuel rod bundles for those cases. None of the calculated $\mathrm{k}_{\mathrm{eff}}$ values for the critical experiments falls below the uniform confidence band $\mathrm{k}_{\mathrm{c}}(\mathrm{x})$-W curve, the lower confidence limit for calculated criticals. The USL value is a minimum at the minimum $\mathrm{H}^{235} \mathrm{U}$ ratio. Extrapolating the USL equation for the $\mathrm{H} /{ }^{235} \mathrm{U}=0$ gives a conservative USL of 0.939 for this trending parameter.

A minimum USL among the results of four trending parameters of 0.934 occurs for both the EALF and the enrichment trending parameters. It is recommended that this USL value be utilized. The method 2 curves in all four trendings verify that the administrative margin is conservative relative to a quantitative statistical estimate (Fig. 3-1). 
Figure 3-1. $k_{\text {eff }}$ Data and the USLSTATS Trending Results

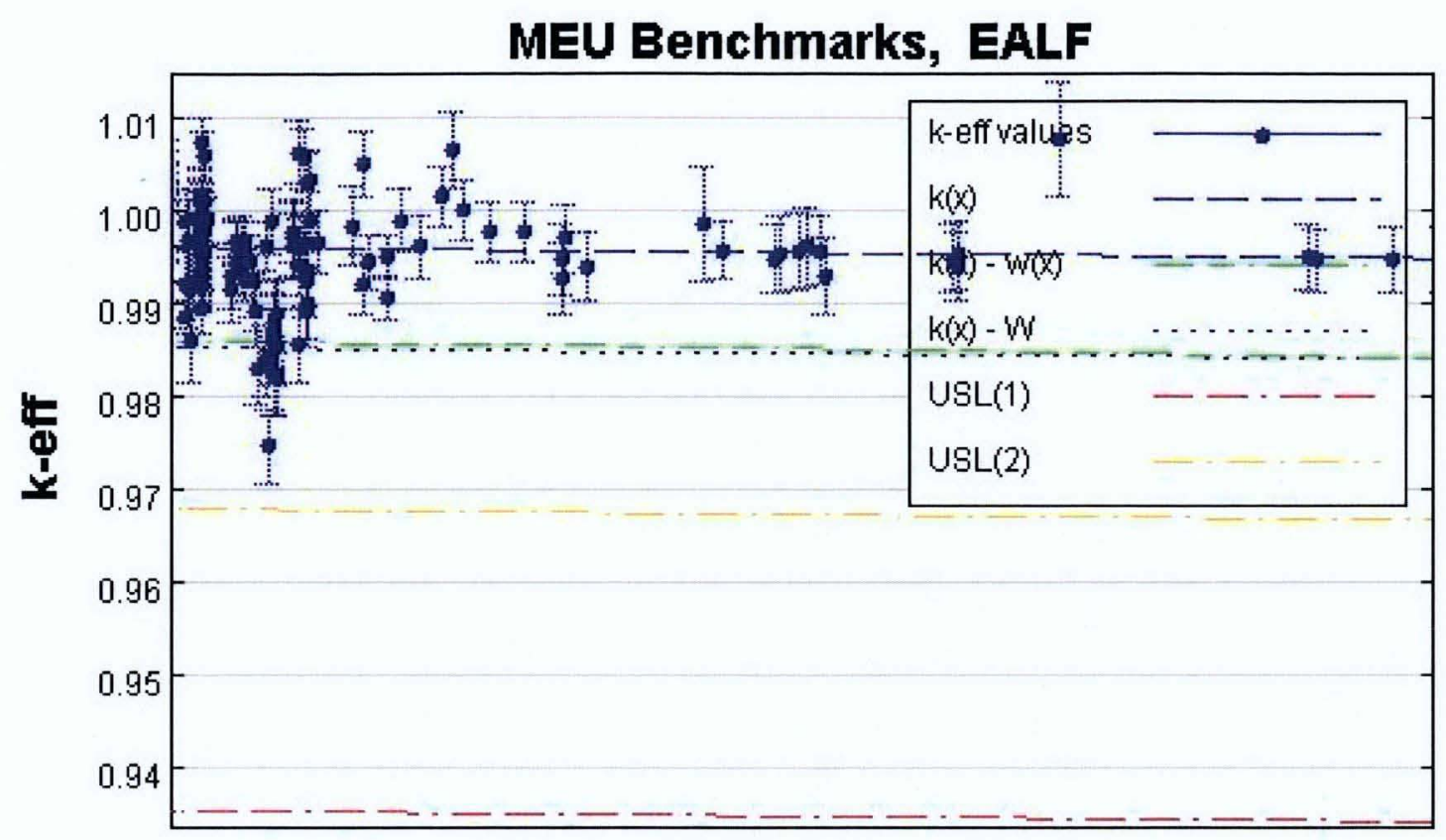

Trending Parameter, $x$ (3.0e-08 - 3.0E-06)

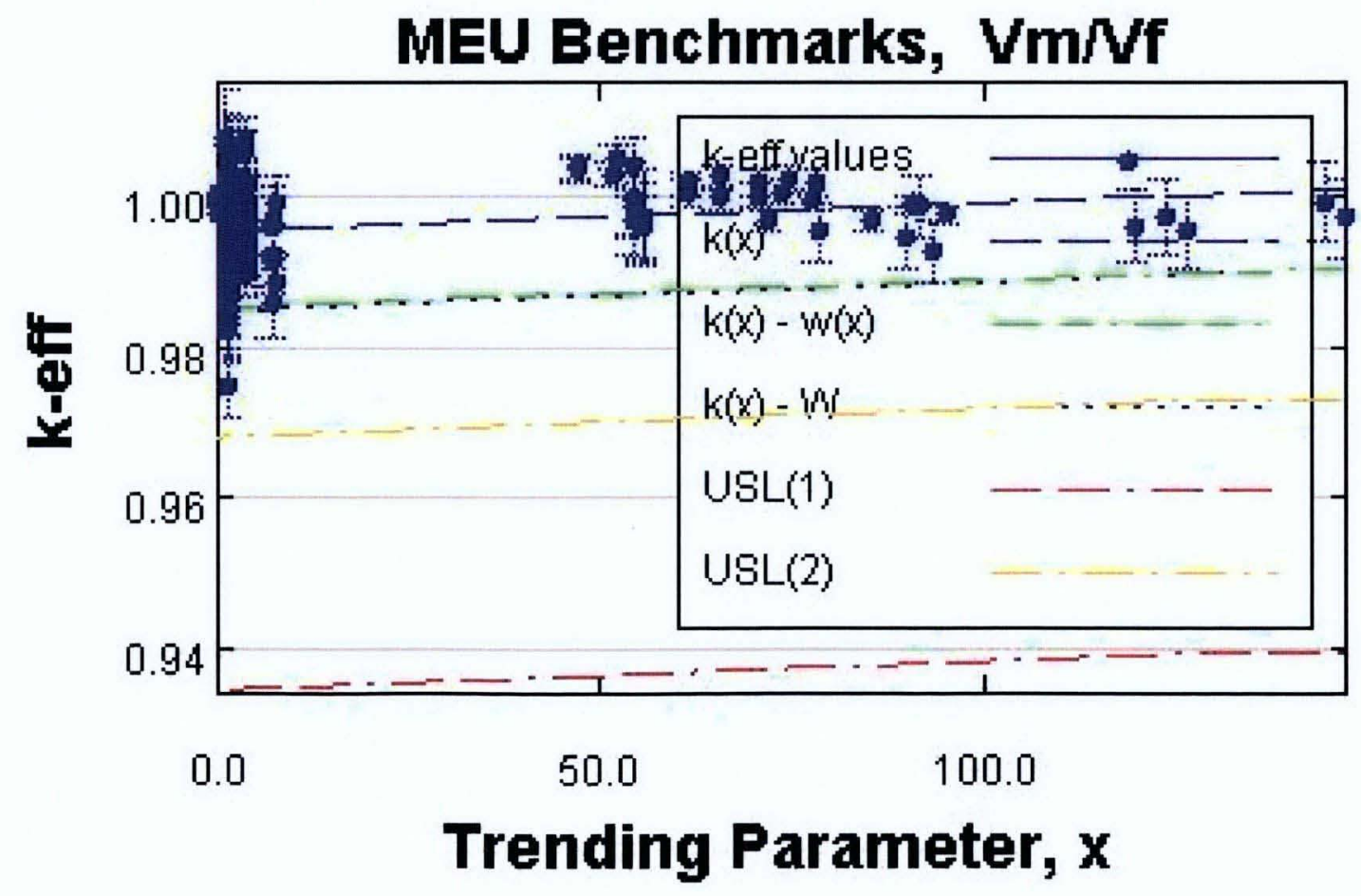



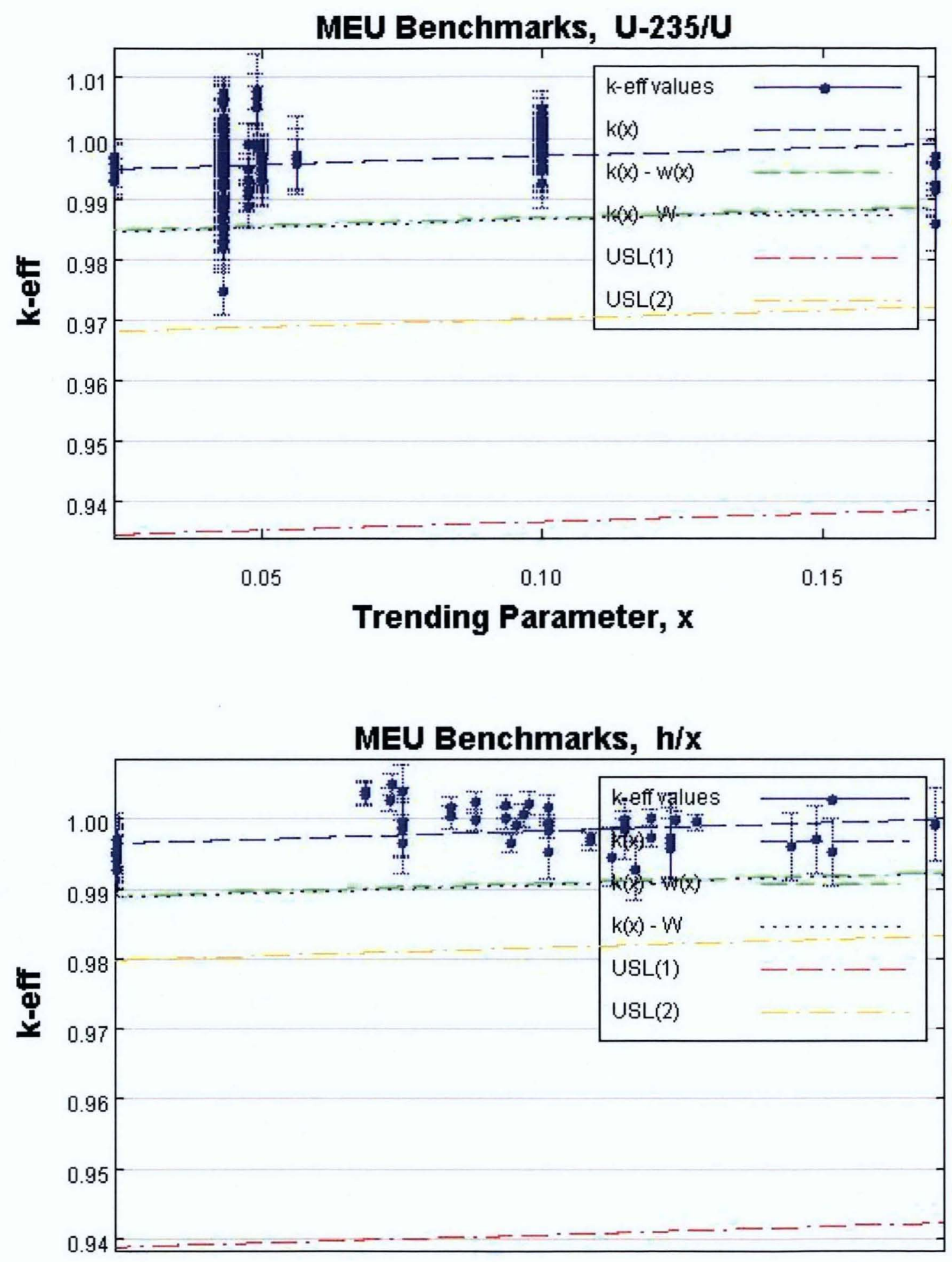

Trending Parameter, $x(35.0-1440.0)$ 


\subsection{DISCUSSION OF CODE AND CROSS SECTIONS USED}

The code used for this validation is MCNP5 Version 1.40. This code is under configuration control. The earlier versions of MCNP have been used extensively for criticality analysis. MCNP is a Monte Carlo code using continuous energy cross sections.

Table B-1 in Appendix B lists the isotope cross sections used in this validation. These are the cross sections that the MCNP5 code uses when an extension (e.g., $.62, .55 \mathrm{c}$ ) is not added to the end of the isotope identifier (e.g., 1001, 94239). No specific selection of cross sections was made because any of the cross section data set included with MCNP code by LANL are considered to be adequate. MCNP will select the first match of an isotope identifier (without extension) found in the directory file XSDIR. This default process has given a cross section set based on ENDF/B-VI when available. It is expected that ENDF/B-VI has a larger number of data points and has corrected data when available. The cross section sets listed in Table B-1 are the only ones validated for calculating $k_{\text {eff }}$ for intermediate enriched uranium by this validation analysis. CSERs that use other cross sections need to address their validity and their affect on the validated USL for the intermediate enriched uranium systems.

\subsection{RECOMMENDED USL}

Based on the data and discussion presented in Section 3 a single USL for the intermediate enriched uranium calculations with the MCNP5 Version 1.40 code of 0.934 is recommended. This conservatively utilizes the minimum USL calculated from all of the four independent parameters. To implement this USL the calculated $\mathrm{k}_{\mathrm{eff}}$ and the statistical uncertainty from future analysis are utilized in the following manner:

$$
\mathrm{k}_{\mathrm{eff}}=\mathrm{k}_{\mathrm{calc}}+1.645 \sigma_{\text {calc }} \leq \mathrm{USL}
$$

where:

$\mathrm{k}_{\mathrm{eff}}=$ the determined $\mathrm{k}$

$\mathrm{k}_{\text {calc }}=$ the MCNP calculated $\mathrm{k}_{\text {eff, }}$

$1.645=$ the number of standard deviations required to achieve $95 \%$ confidence in the calculation for a one sided distribution,

$\sigma_{\text {calc }}=$ the MCNP calculated statistical uncertainty, and

$\mathrm{USL}=0.934$.

This USL assures that future analyses of intermediate enriched uranium systems will be safely subcritical if the MCNP calculated $\mathrm{k}_{\text {eff }}$ and statistical uncertainty meets the above criteria.

This can also be implemented via a calculational bias. A USL of 0.934 implies a bias of 0.016. This is implemented in the equation:

$$
\mathrm{k}_{\text {calc }}=\mathrm{k}_{\text {limit }}+\text { bias }-1.645 \sigma_{\text {calc }}
$$

where:

$$
\mathrm{k}_{\mathrm{calc}}=\text { the MCNP calculated } \mathrm{k}_{\mathrm{eff}} \text {, }
$$


$\mathrm{k}_{\text {limit }}=0.95$, bias $=0.016$,

$1.645=$ the number of standard deviations required to achieve $95 \%$ confidence in the calculation for a one sided distribution, and,

$\sigma_{\text {calc }}=$ the MCNP calculated statistical uncertainty.

When the MCNP calculated statistical uncertainty is $<0.001$ this gives the recommended $k_{\text {eff }}$ value of 0.932 for the IEU systems.

When the analyst is utilizing the above USL data, for a specific analysis, they will need to determine that this evaluation is sufficient for their needs. Where applicable, they should discuss whether their specific application is adequately within the various ranges of applicability for the different trending parameters and that isotopic cross sections validated in this analysis were used, and if not, the justification for their use. Or if outside some of the ranges, discuss why this validation data and USL analysis is adequate.

\subsection{REFERENCES}

LA-UR-03-1987, 2005, MCNP - A General Monte Carlo N-Particle Transport Code, Version 5 1.40, Los Alamos National Laboratory, Los Alamos, New Mexico.

Lan, J. S., 2005, Requirements and Management Plan, Use of MCNP Version 4C for Safety Significant and Safety Class Applications at Fluor Hanford, HNF-26564, Rev. 0A, Fluor Hanford, Inc., Richland, Washington.

NEA/NSC/DOC(95)03, 2005, International Handbook Of Evaluated Criticality Safety

Benchmark Experiments, Volume VI, , Plutonium Systems, Nuclear Energy Agency, Paris, France.

ORNL, 1998, USLSTATS: A Utility to Calculate Upper Subcritical Limits for Criticality Safety Applications, Oak Ridge National Laboratory, Oak Ridge, Tennessee. 
CHPRC-00455, REV. 0

APPENDIX A - TECHNICAL PEER REVIEW

A-1 
CHPRC-00455, REV. 0

This page is intentionally left blank 


\section{Peer Review Comments}

This validation report for Intermediate Enriched Uranium compounds was independently reviewed by E. M. Miller, a qualified Criticality Safety Specialist in the Criticality and Shielding group of Fluor Government Group. The reviewer concurs with the USL value determined in the report and offers the following review in support of this conclusion.

The USLSTATS statistical program for analyzing criticality validation data assumes that the data points have a normal distribution. As part of the statistical program's analysis, the data is tested for normality. The three of the four trending runs of the statistics program in this validation analysis failed its crude test method. If the data does not have a normal distribution, the confidence band calculated by the program may not be as large as the statistics of the data would warrant. The data could be tested for normality by more sophisticated methods to justify using the margins calculated by the program. Instead, the visual and historical analyses of the data will be used to show that the large margin of safety in the USL value is adequate.

The average of the 167 data points is 0.996 as shown in Appendix D. The standard deviation is 0.0062 . The validation cases show that MCNP calculates the $k_{\text {eff }}$ accurately with a small deviation.

Looking at the margin between the USL(1) line (Confidence band for a single future calculation with administrative margin) and the lower confidence interval (one-sided, uniform-width, lower 95.0 confidence band for a single future calculation) in Figure 3-1, one can intuitively see that there is an adequate margin for the data points being analyzed. Further, since the $\mathrm{k}(\mathrm{x})-\mathrm{W}(\mathrm{x})$ and $\mathrm{k}(\mathrm{x})-\mathrm{W}$ curves are parallel and the first is not noticeably parabolic in shape over the trending parameter's range, the confidence band is not significantly dependent on the trending parameter. Without the margin being dependent on the trending parameter and the average $k_{\text {eff }}$ being approximately 1.0 , the variance of the $\mathrm{k}_{\mathrm{eff}} \mathrm{S}$ about its mean can be used to estimate a conservative confidence band.

Appendix D gives the pooled standard deviation (variance of the linear regression fit of the calculated $k_{\text {eff }}$ values and the statistical variance of each calculated $k_{\text {eff }}$ value) to use. This value needs to be multiplied by a statistical distribution value that incorporates the number of experiments (167 data points), the expected confidence level ( 0.95 confidence that $99.5 \%$ of the data points are above the lower tolerance interval), a one sided distribution, and the distribution of data points. The Student-T one sided distribution value specified in Appendix D by USLSTATS is 1.645 . The USLSTATS program multiplies this number by a value of 1.0438 to give a multiplier of 1.72 . As a conservatism, a multiplier of 2.0 is used here.

All pooled standard deviations calculated for the trend cases were less than 0.0062 . Using the multiplier of 2.0 from above, the confidence band would be 0.012 . Subtracting this from the average $\mathrm{k}_{\text {eff }}$ value of 0.996 gives a value of 0.984 . Reducing this result by our administrative margin of 0.05 gives 0.934 . This agrees with the 0.934 minimum value for USL(1) in Table 3-1. Based on this result and the visual margin found in the Figures 3-1, this reviewer considers the USL value of 0.934 to be adequately conservative for Intermediate Enriched Uranium compounds. 
CHPRC-00455, REV. 0

\section{CHECKLIST FOR TECHNICAL PEER REVIEW}

Document Reviewed - HNF-30616, Rev. 0

Title: MCNP5 Criticality Validation and Bias for Intermediate Enriched Uranium Systems

Author(s): J.S.Lan

Date: May 2007

Scope of Review: Entive docoment ekcept MCNP runs.

\begin{tabular}{|c|c|c|c|c|}
\hline Yes & No* & NA & & \\
\hline & [ ] & [ ] & & Referenced analyses appropriate. \\
\hline & [ ] & [ ] & & $\begin{array}{l}\text { Problem completely defined and all potential configurations } \\
\text { considered. }\end{array}$ \\
\hline ] & ] & 54 & & Accident scenarios developed in a clear and logical manner. \\
\hline & [ ] & [ ] & & Necessary assumptions explicitly stated and supported. \\
\hline & [ ] & [ & & Computer codes and data files documented. \\
\hline & ] & ] & & Data used in calculations explicitly stated in document. \\
\hline & [ ] & {[} & & $\begin{array}{l}\text { Data checked for consistency with original source information as } \\
\text { applicable. }\end{array}$ \\
\hline & [ ] & & & $\begin{array}{l}\text { Mathematical derivations checked including dimensional } \\
\text { consistency of results }\end{array}$ \\
\hline & ] & {[} & & $\begin{array}{l}\text { Models appropriate and used within range of validity, or use outside } \\
\text { range of established validity justified. }\end{array}$ \\
\hline & [ & [ & & $\begin{array}{l}\text { Hand calculations checked for errors. Spreadsheet results should be } \\
\text { treated exactly the same as hand calculations. }\end{array}$ \\
\hline [ & [ ] & $\infty$ & & Software input correct and consistent with document reviewed. \\
\hline & [ & $\infty$ & & $\begin{array}{l}\text { Software output consistent with input and with results reported in } \\
\text { document reviewed. }\end{array}$ \\
\hline & 了 & {[} & & $\begin{array}{l}\text { Limits/criteria/guidelines applied to analysis results are appropriate } \\
\text { and referenced. Limits/criteria/guidelines checked against } \\
\text { references. }\end{array}$ \\
\hline & ] & [ & & Safety margins consistent with good engineering practices. \\
\hline & ] & {[} & & Conclusions consistent with analytical results and applicable limits. \\
\hline & [ & & & $\begin{array}{l}\text { Results and conclusions address all points required in the problem } \\
\text { statement. }\end{array}$ \\
\hline & ] & & & Format consistent with applicable guides or other standards. \\
\hline & ] & & ** & Review calculations, comments, and/or notes are attached. \\
\hline & ] & [ ] & & $\begin{array}{l}\text { Document approved (for example, the reviewer affirms the technical } \\
\text { accuracy of the document). }\end{array}$ \\
\hline
\end{tabular}

E. M. Miller Elumad II Inder 23 MAY 2007

Technical Peer Reviewer (printed name and signature) Date

* All "no" responses must be explained below or on an additional sheet.

* Any calculations, comments, or notes generated as part of this review should be signed, dated and attached to this checklist. The material should be labeled and recorded in such a manner as to be understandable to a technically qualified third party. 


\section{Checklist For Technical Peer Review}

Document Reviewed - HNF-30616, Rev. 0

Title: MCNP5 Criticality Validation and Bias for Intermediate Enriched Uranium Systems

Author: J. S. Lan

Date: August 2006

Reviewed: MEU Calculations

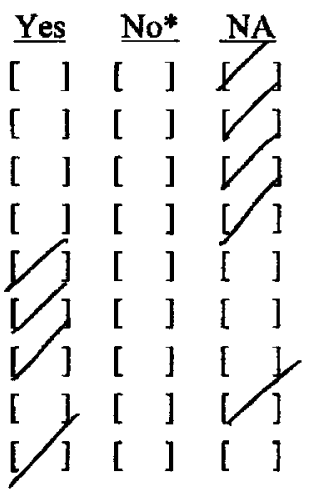

\section{$\left[\begin{array}{lllll} & {[} & {[} & 1\end{array}\right]$}
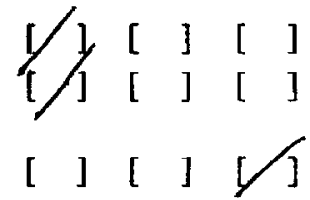

[ ] [ ]

[ ] [ ]

[ ] [ ]

[ ] [ ] $1 / 7$

[ ] [ ] []

[ $[1][]$
Referenced analyses appropriate.

Problem completely defined and all potential configurations considered.

Accident scenarios developed in a clear and logical manner.

Necessary assumptions explicitly stated and supported.

Computer codes and data files documented.

Data used in calculations explicitly stated in document.

Data checked for consistency with original source information as applicable.

Mathematical derivations checked including dimensional consistency of results.

Models appropriate and used within range of validity, or use outside range of established validity justified.

Hand calculations checked for errors. Spreadsheet results should be treated exactly the same as hand calculations.

Software input correct and consistent with document reviewed.

Software output consistent with input and with results reported in document reviewed.

Limits/criteria/guidelines applied to analysis results are appropriate and referenced. Limits/criteria/guidelines checked against references.

Safety margins consistent with good engineering practices.

Conclusions consistent with analytical results and applicable limits.

Results and conclusions address all points required in the problem statement.

Format consistent with applicable guides or other standards.

** Review calculations, comments, and/or notes are attached.

Document approved (for example, the reviewer affirms the technical accuracy of the document).

\section{SR Gedeon $N$}

Technical Peer Reviewer (printed name and signature)

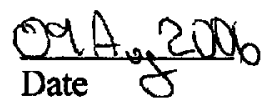

- All "no" responses must be explained below or on an additional sheet.

* Any calculations, comments, or notes generated as part of this review should be signed, dated and attached to this checklist. The material should be labeled and recorded in such a manner as to be understandable to a technically qualified third party. 
CHPRC-00455, REV. 0

This page is intentionally left blank 
CHPRC-00455, REV. 0

APPENDIX B - TABLE OF CROSS-SECTIONS UTILIZED 
CHPRC-00455, REV. 0

This page is intentionally left blank 
CHPRC-00455, REV. 0

\section{Table B-1. MCNP Cross Section ID's Used in This Analysis}

\begin{tabular}{|r|l|l|r|}
\hline $\mathrm{Z}$ & \multicolumn{2}{|c|}{ Isotope } & MCNP ID \\
\hline 1 & $\mathrm{H}$ & Hydrogen & $1001.62 \mathrm{c}$ \\
\hline 5 & $\mathrm{~B}$ & Boron & $5010.66 \mathrm{c}$ \\
\hline & & & $5011.66 \mathrm{c}$ \\
\hline 6 & $\mathrm{C}$ & Carbon & $6000.66 \mathrm{c}$ \\
\hline 7 & $\mathrm{~N}$ & Nitrogen & $7014.62 \mathrm{c}$ \\
\hline 8 & $\mathrm{O}$ & Oxygen & $8016.62 \mathrm{c}$ \\
\hline 12 & $\mathrm{Mg}$ & Magnesium & $12000.62 \mathrm{c}$ \\
\hline 13 & $\mathrm{Al}$ & Aluminum & $13027.62 \mathrm{c}$ \\
\hline 14 & $\mathrm{Si}$ & Silicon & $14000.60 \mathrm{c}$ \\
\hline 15 & $\mathrm{P}$ & Phosphorus & $15031.66 \mathrm{c}$ \\
\hline 16 & $\mathrm{~S}$ & Sulfur & $16000.62 \mathrm{c}$ \\
\hline & & & $16032.62 \mathrm{c}$ \\
\hline 20 & $\mathrm{Ca}$ & Calcium & $20000.62 \mathrm{c}$ \\
\hline
\end{tabular}

\begin{tabular}{|r|l|l|r|}
\hline $\mathrm{Z}$ & \multicolumn{2}{|c|}{ Isotope } & MCNP ID \\
\hline 22 & $\mathrm{Ti}$ & Titanium & $22000.62 \mathrm{c}$ \\
\hline 24 & $\mathrm{Cr}$ & Chromium & $24000.50 \mathrm{c}$ \\
\hline 25 & $\mathrm{Mn}$ & Manganese & $25055.62 \mathrm{c}$ \\
\hline 26 & $\mathrm{Fe}$ & Iron & $26000.55 \mathrm{c}$ \\
\hline 28 & $\mathrm{Ni}$ & Nickel & $28000.50 \mathrm{c}$ \\
\hline 29 & $\mathrm{Cu}$ & Copper & $29000.50 \mathrm{c}$ \\
\hline 40 & $\mathrm{Zr}$ & Zirconium & $40000.66 \mathrm{c}$ \\
\hline 41 & $\mathrm{Nb}$ & Niobium & $41093.66 \mathrm{c}$ \\
\hline 42 & $\mathrm{Mo}$ & Molybdenum & $42000.66 \mathrm{c}$ \\
\hline 64 & $\mathrm{Gd}$ & Gadolinium & $64152.66 \mathrm{c}$ \\
\hline & & & $64154.66 \mathrm{c}$ \\
\hline & & & $64155.66 \mathrm{c}$ \\
\hline & & & $64156.66 \mathrm{c}$ \\
\hline
\end{tabular}

\begin{tabular}{|r|l|l|r|}
\hline $\mathrm{Z}$ & \multicolumn{2}{|c|}{ Isotope } & MCNP ID \\
\hline & & & $64157.66 \mathrm{c}$ \\
\hline & & & $64158.66 \mathrm{c}$ \\
\hline & & & $64160.66 \mathrm{c}$ \\
\hline 67 & Ho & Holmium & $67165.66 \mathrm{c}$ \\
\hline 72 & $\mathrm{Hf}$ & Hofnium & $72000.60 \mathrm{c}$ \\
\hline 82 & $\mathrm{~Pb}$ & Lead & $82000.50 \mathrm{c}$ \\
\hline 92 & $\mathrm{U}$ & Uranium & $92234.69 \mathrm{c}$ \\
\hline & & & $92235.69 \mathrm{c}$ \\
\hline & & & $92236.69 \mathrm{c}$ \\
\hline & & & $92238.69 \mathrm{c}$ \\
\hline
\end{tabular}

Notes: MCNP IDs that end in $.50 \mathrm{c}$ or $.55 \mathrm{c}$ utilize ENDF/B-V cross sections. MCNP IDs that end in $.60 \mathrm{c}, .62 \mathrm{c}$, $.66 \mathrm{c}$ utilize ENDF/B-VI cross sections. MCNP IDs that end in .69c utilize ENDF/B-VII cross-sections. 


\section{CHPRC-00455, REV. 0}

This page is intentionally left blank 
CHPRC-00455, REV. 0

APPENDIX C - MCNP INPUT FILES

C-1 
CHPRC-00455, REV. 0

This page is intentionally left blank 


\section{CHPRC-00455, REV. 0}

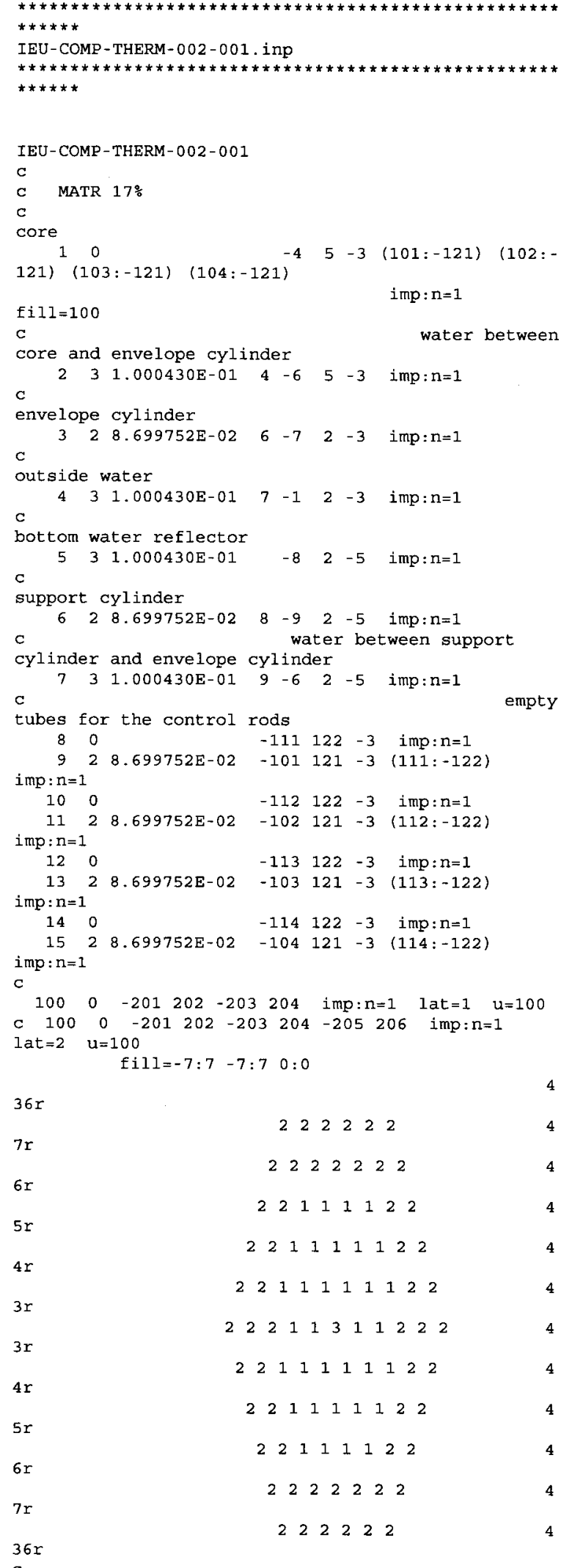

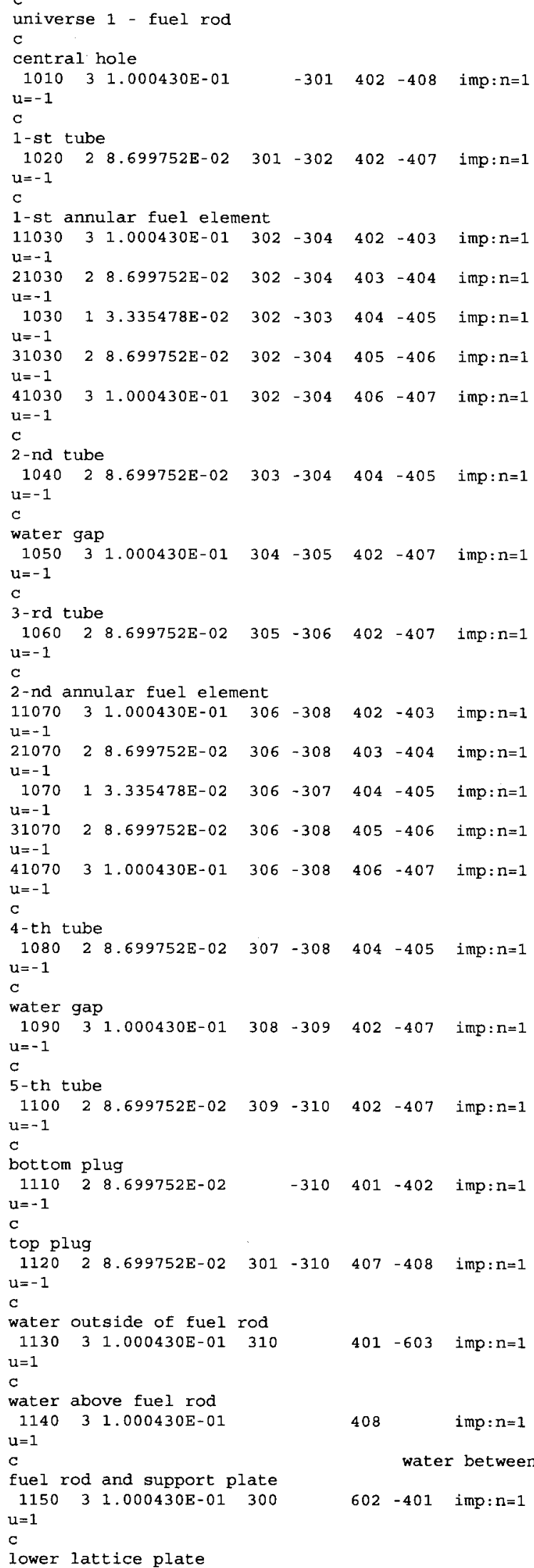




\section{CHPRC-00455, REV. 0}

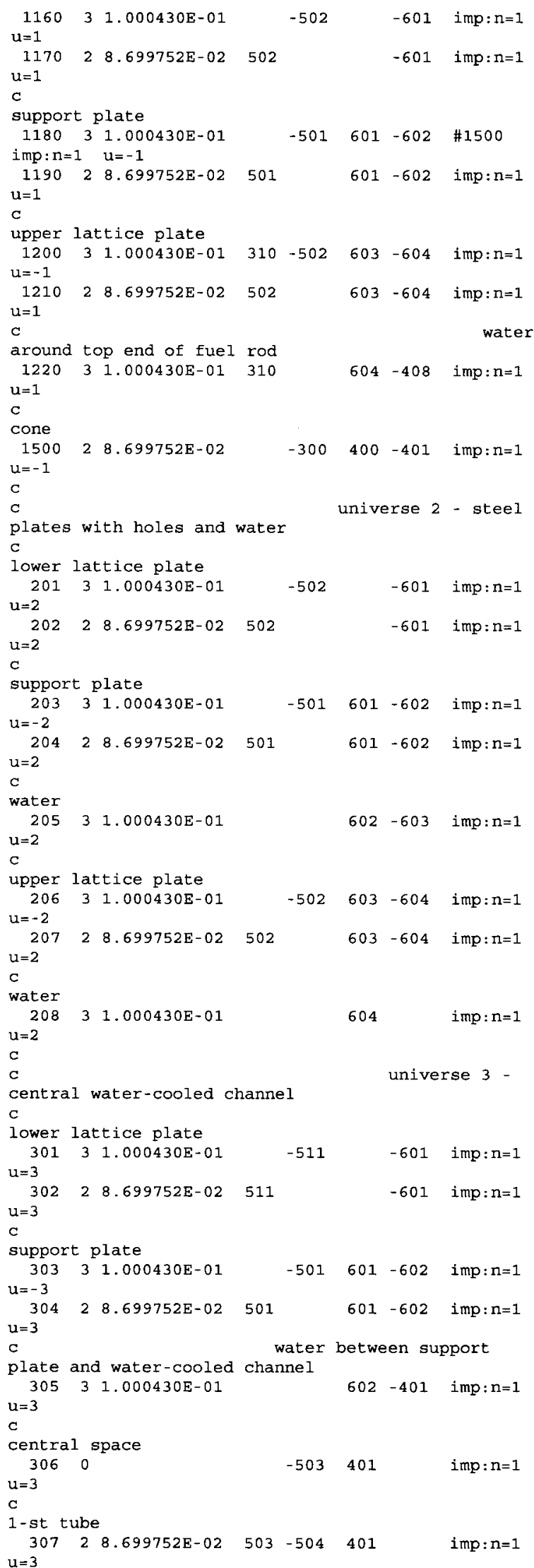




\section{CHPRC-00455, REV. 0}

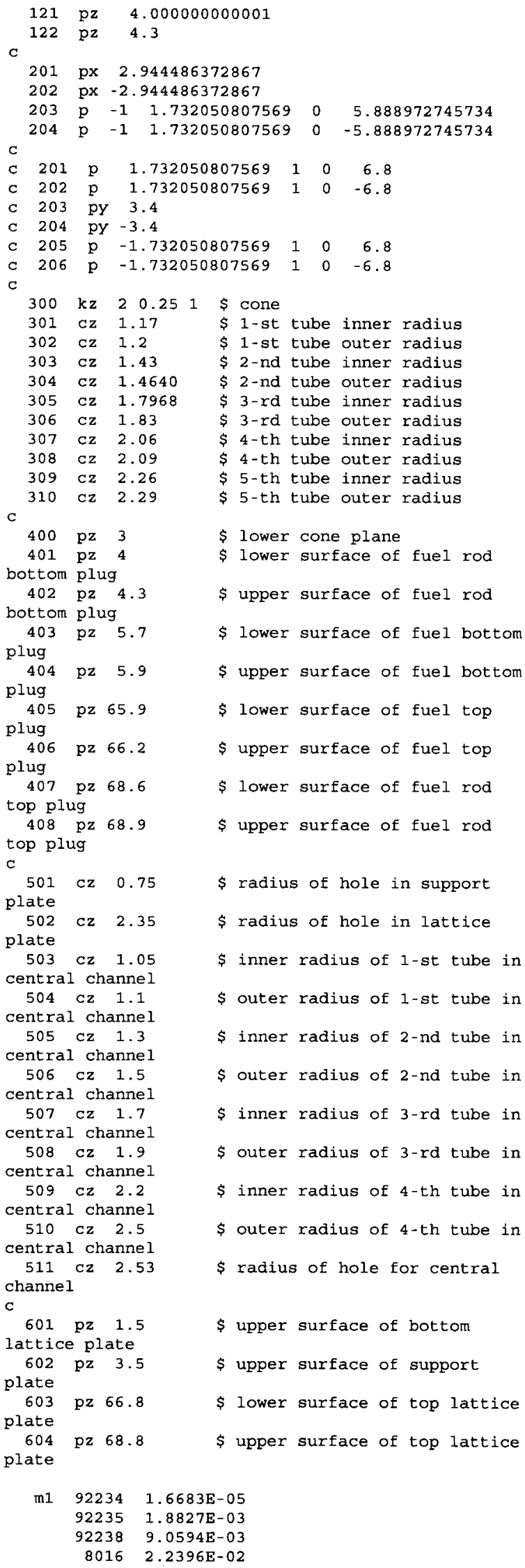

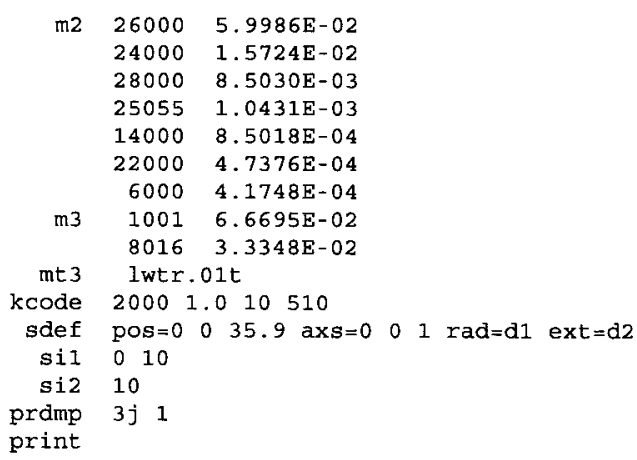




\section{CHPRC-00455, REV. 0}

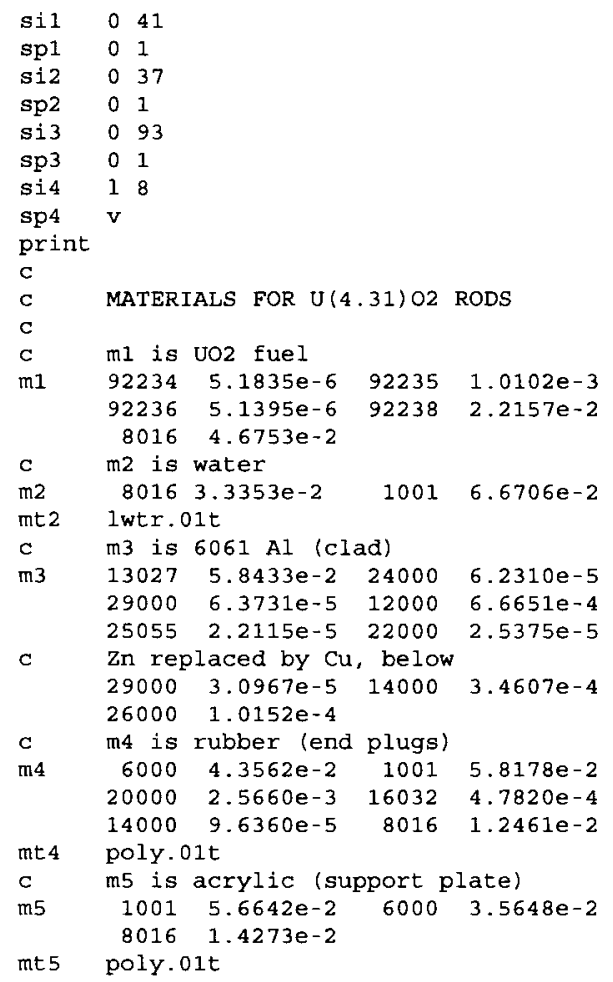

11 px .0001 \$ closest edge of closest cluster

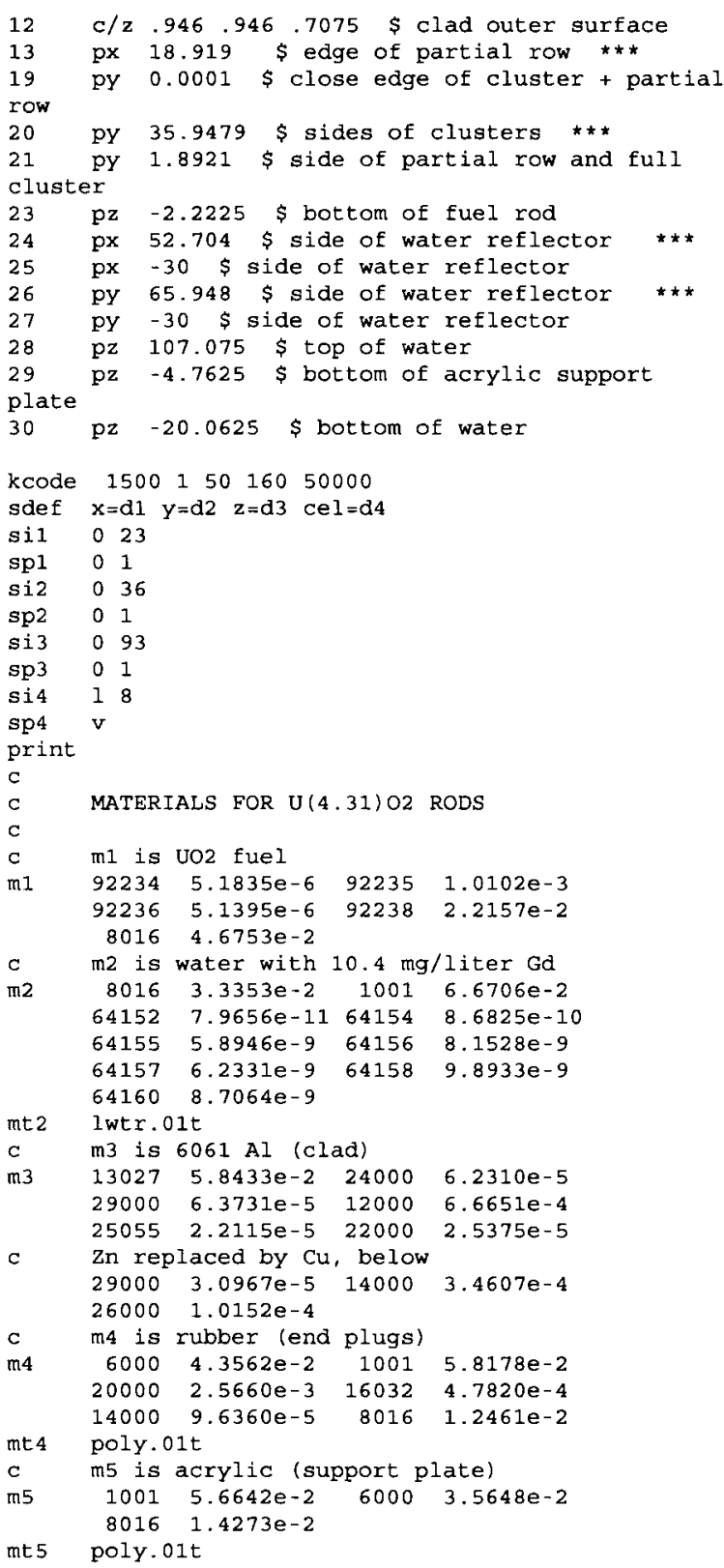

M401p THREE 15X8 CLUSTERS OF U(4.306)O2 RODS, 2.540 CM PITCH, 8.58 CM SEP

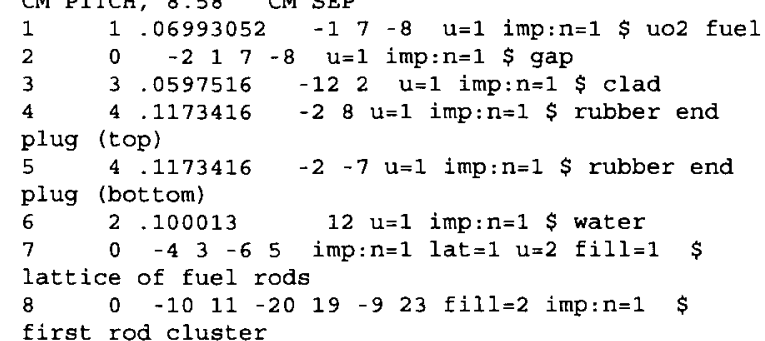




\section{CHPRC-00455, REV. 0}

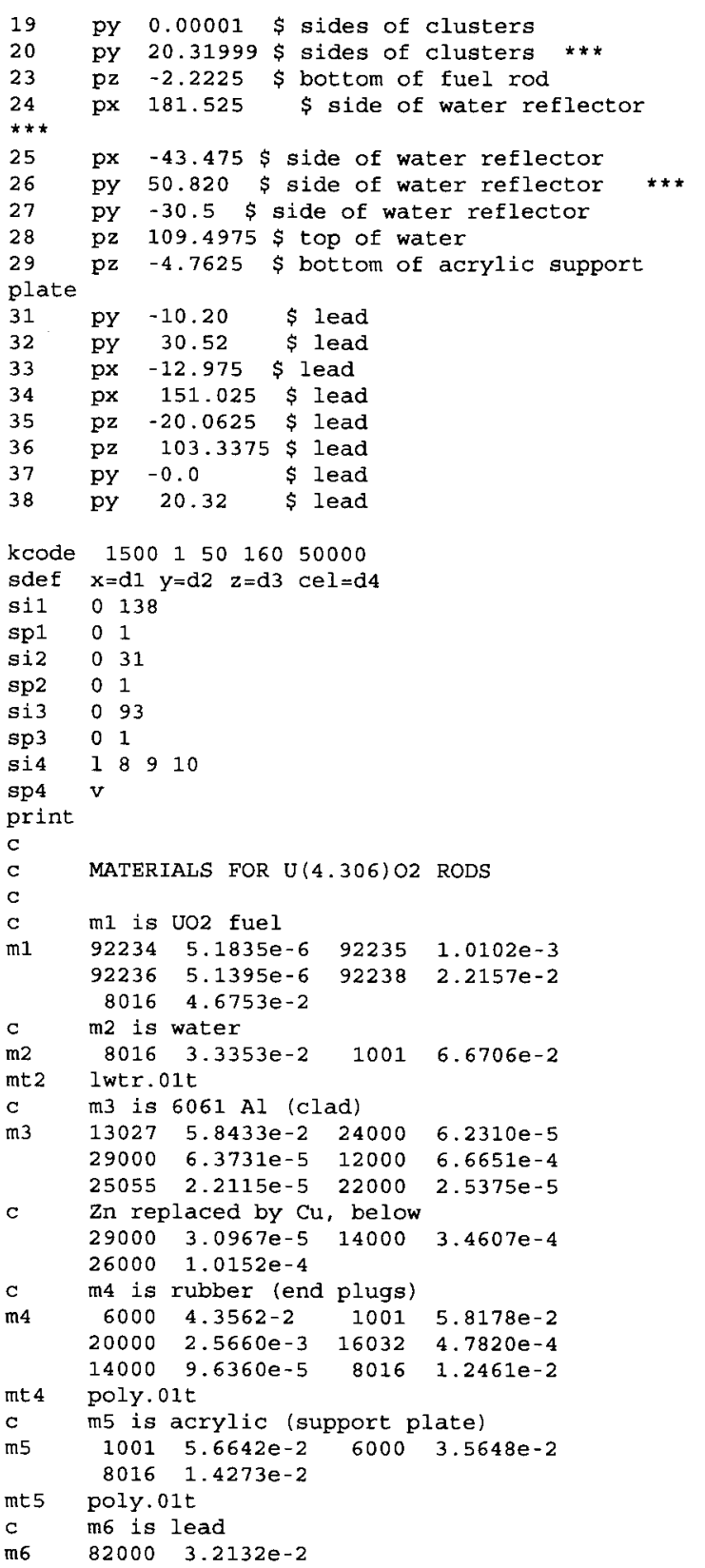

\section{$\star \star \star \star * * *$ \\ LEU-COMP-THERM-026-001. inp

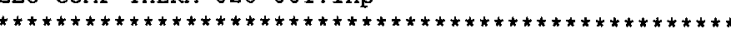

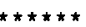

LEU-COMP-THERM-026-001

C MATR $12.9 \mathrm{~mm}$

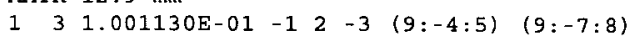
(101:-102:103:-104:105:-106:-

$5: 6)$ imp: $n=1$

$20-101102-103104-1051065-6$ imp: $n=1$ fill $=2$

$3 \quad 3 \quad 1.001130 E-01-201202 \quad-203 \quad 204 \quad-205 \quad 206$

imp: $n=1 \quad$ lat $=2 \quad u=2$

fill $=-15: 15-15: 15 \quad 0: 0$

$245 r$

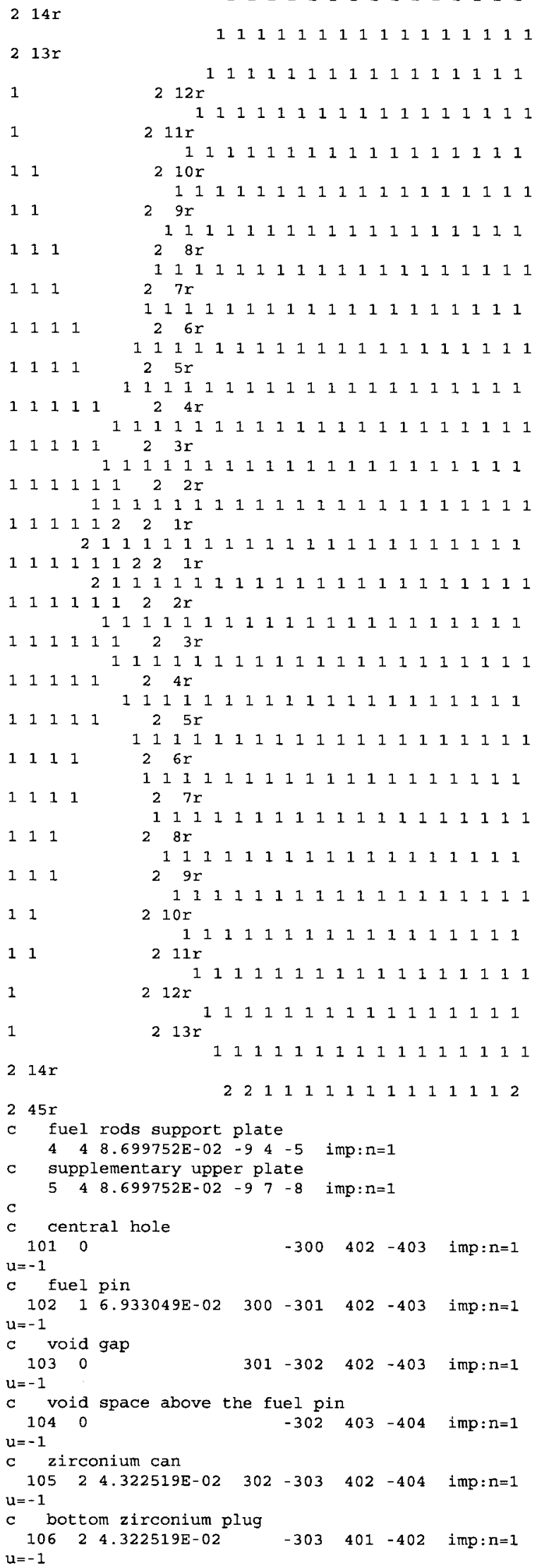




\section{CHPRC-00455, REV. 0}

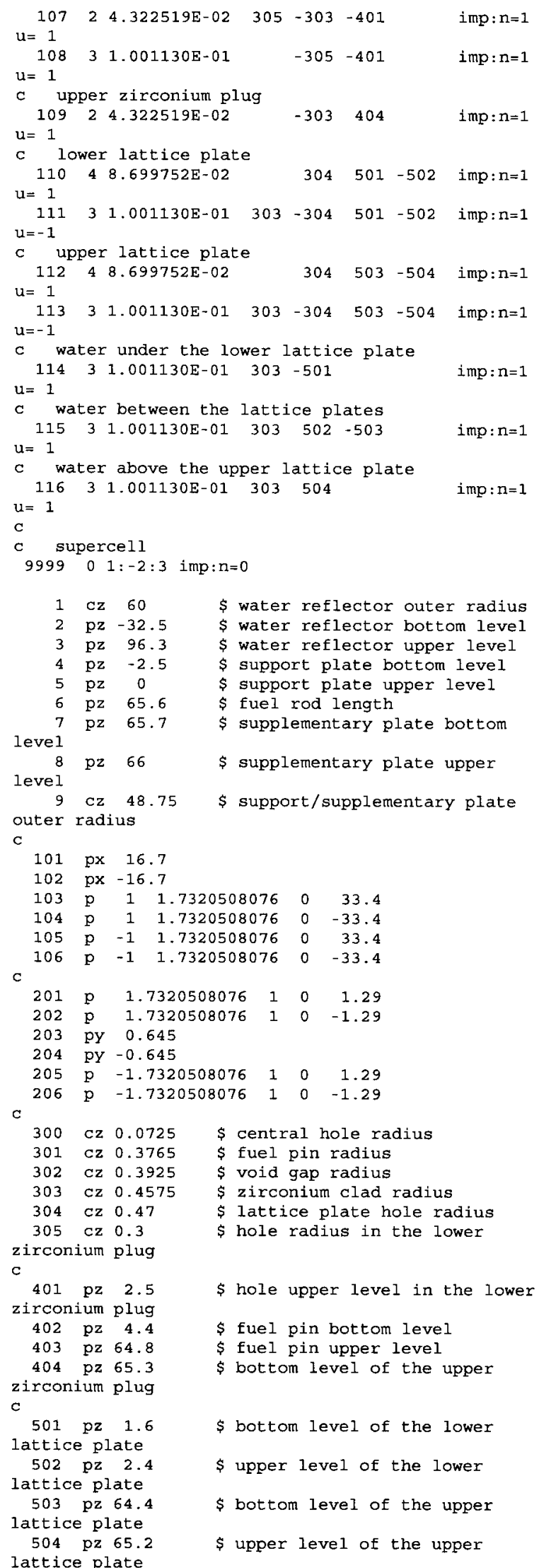

$92234 \quad 4.5882 \mathrm{E}-06$

$922351.1239 \mathrm{E}-03$

$922382.1441 \mathrm{E}-02$

$80164.6761 \mathrm{E}-02$

c Cross section in material 2 was changed from $50 \mathrm{C}$ to $60 \mathrm{C}$

m2 $40000 \quad 4.2794 \mathrm{E}-02$

$41093 \quad 4.2456 \mathrm{E}-04$

$72000 \quad 6.6297 \mathrm{E}-06$

m3 $1001 \quad 6.6742 \mathrm{E}-02$

$8016 \quad 3.3371 \mathrm{E}-02$

mt.3 1wtr.01t

m4 $26000 \quad 5.9986 E-02$

$24000 \quad 1.5724 \mathrm{E}-02$

$28000 \quad 8.5030 \mathrm{E}-03$

$250551.0431 \mathrm{E}-03$

$14000 \quad 8.5018 \mathrm{E}-04$

$22000 \quad 4.7376 \mathrm{E}-04$

$6000 \quad 4.1748 \mathrm{E}-04$

kcode $20001.0 \quad 10 \quad 510$

sdef $\operatorname{pos}=0 \quad 0 \quad 34.6$ axs $=0 \quad 0 \quad 1 \quad \mathrm{rad}=\mathrm{d} 1 \mathrm{ext}=\mathrm{d} 2$

sil $0 \quad 16.8782$

si2 30.2

prdmp $3 j 1$

print

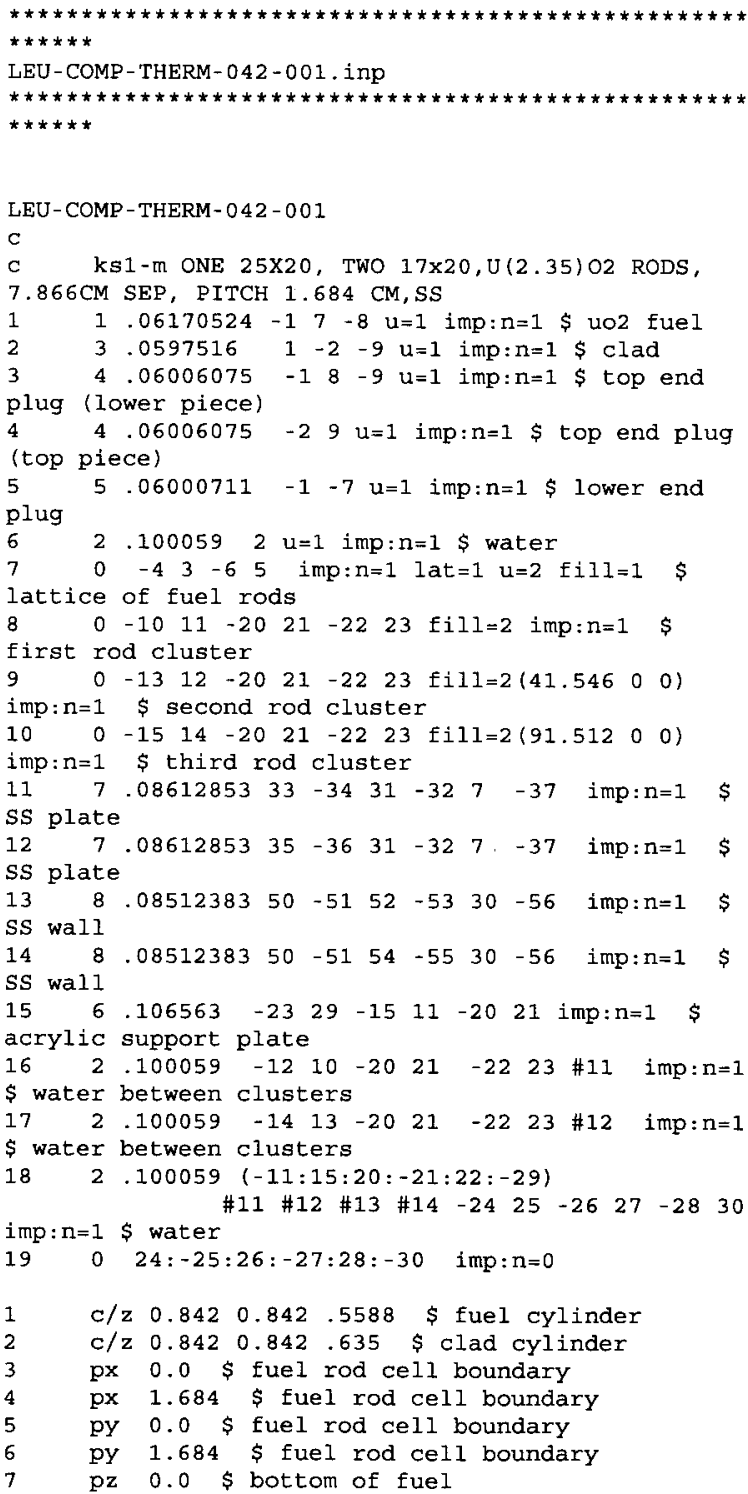




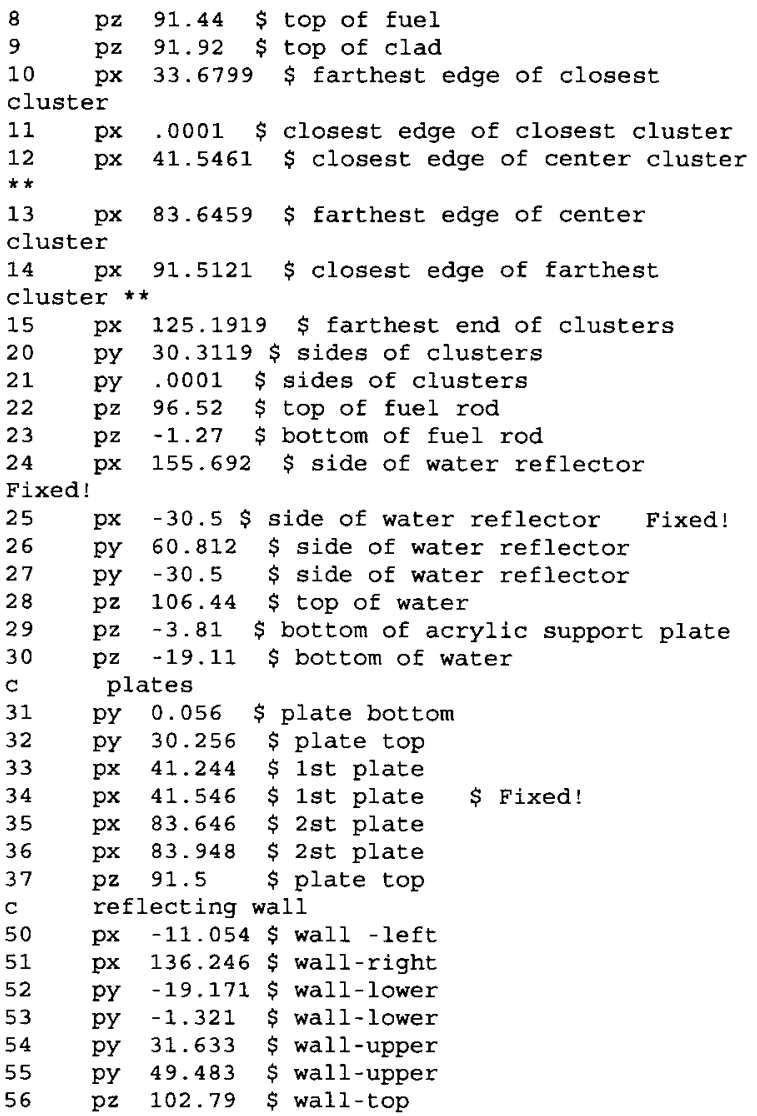

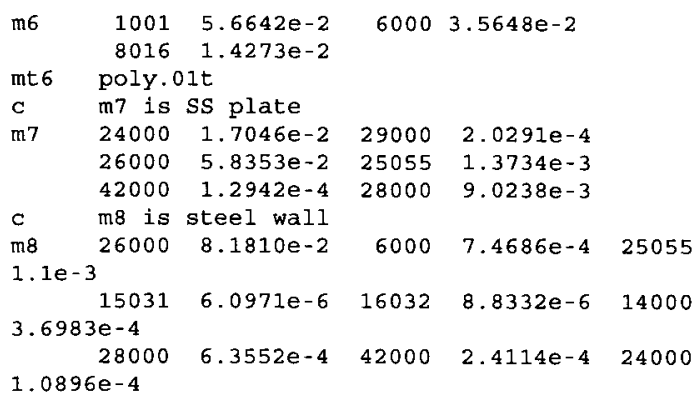$$
\begin{array}{lllllllll} 
& 1.4396547 e-1 & 24 & -25 & 6 & -5 & 8 & -7 & (30:-31: 32:-
\end{array}
$$ 


\section{CHPRC-00455, REV. 0}
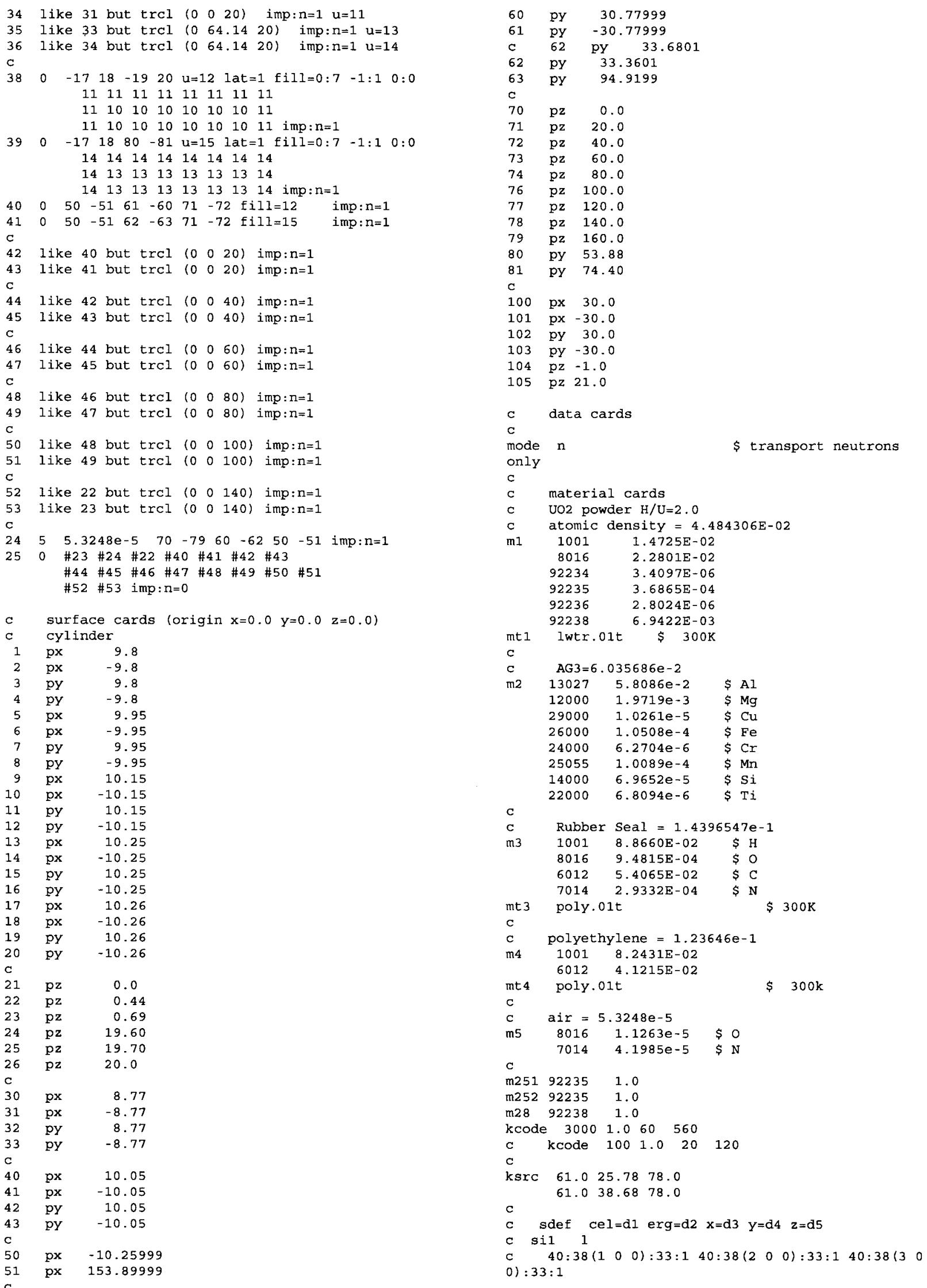


\section{CHPRC-00455, REV. 0}

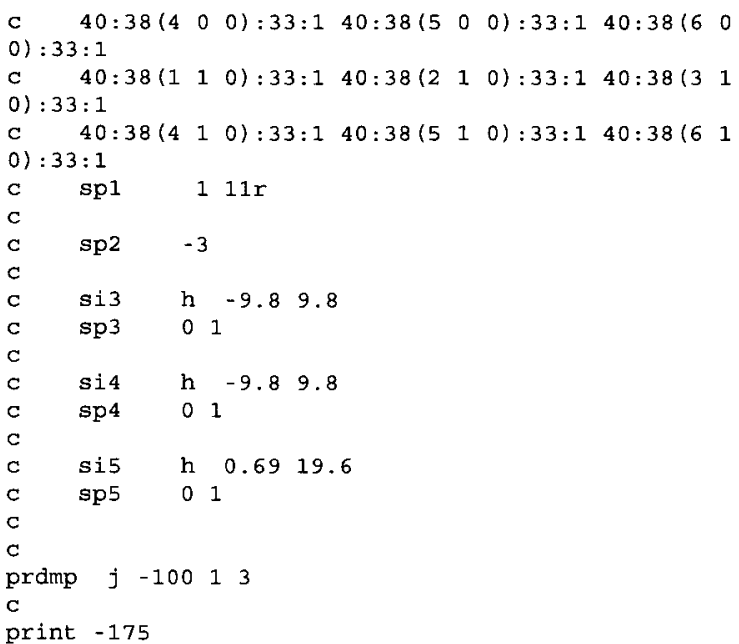

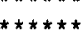 \\ LEU-COMP - THERM- 052 - 001 . inp

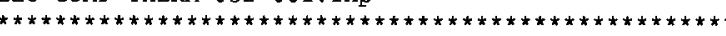
$\star * * * * *$

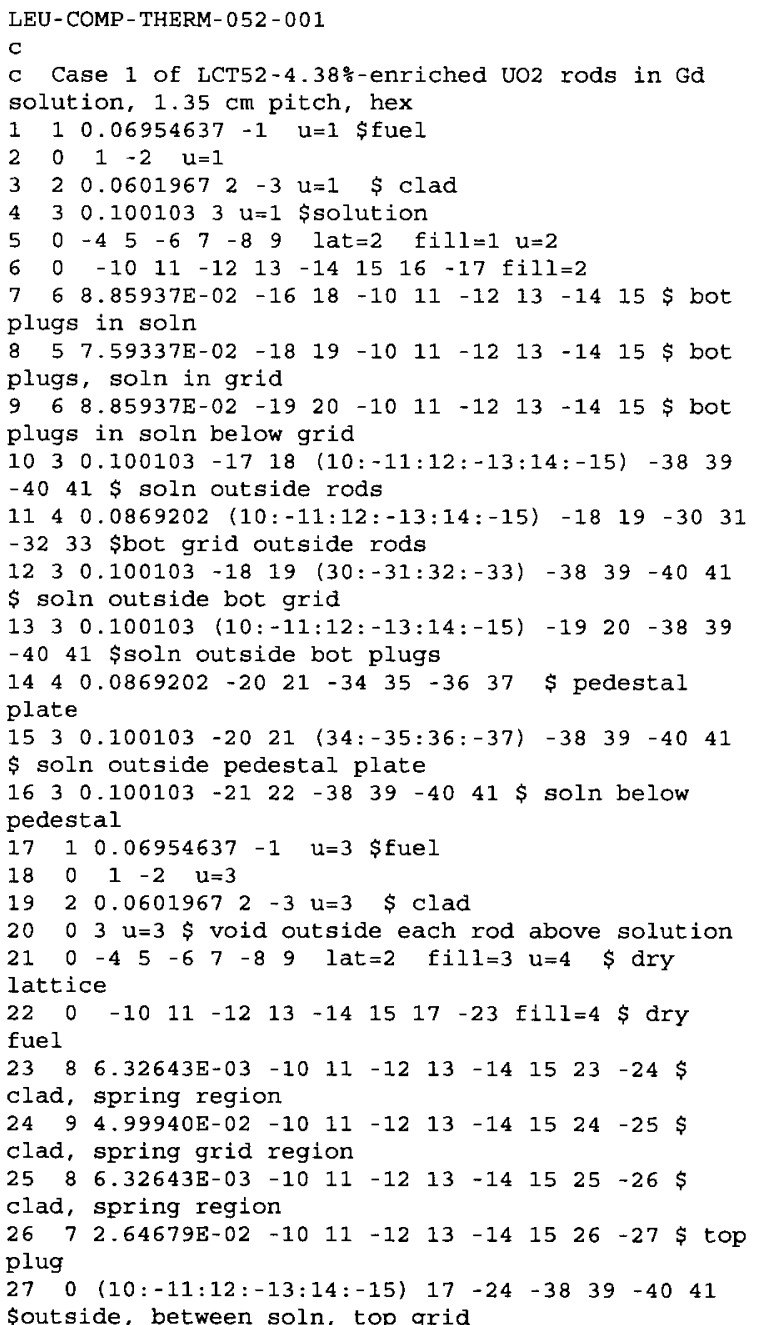

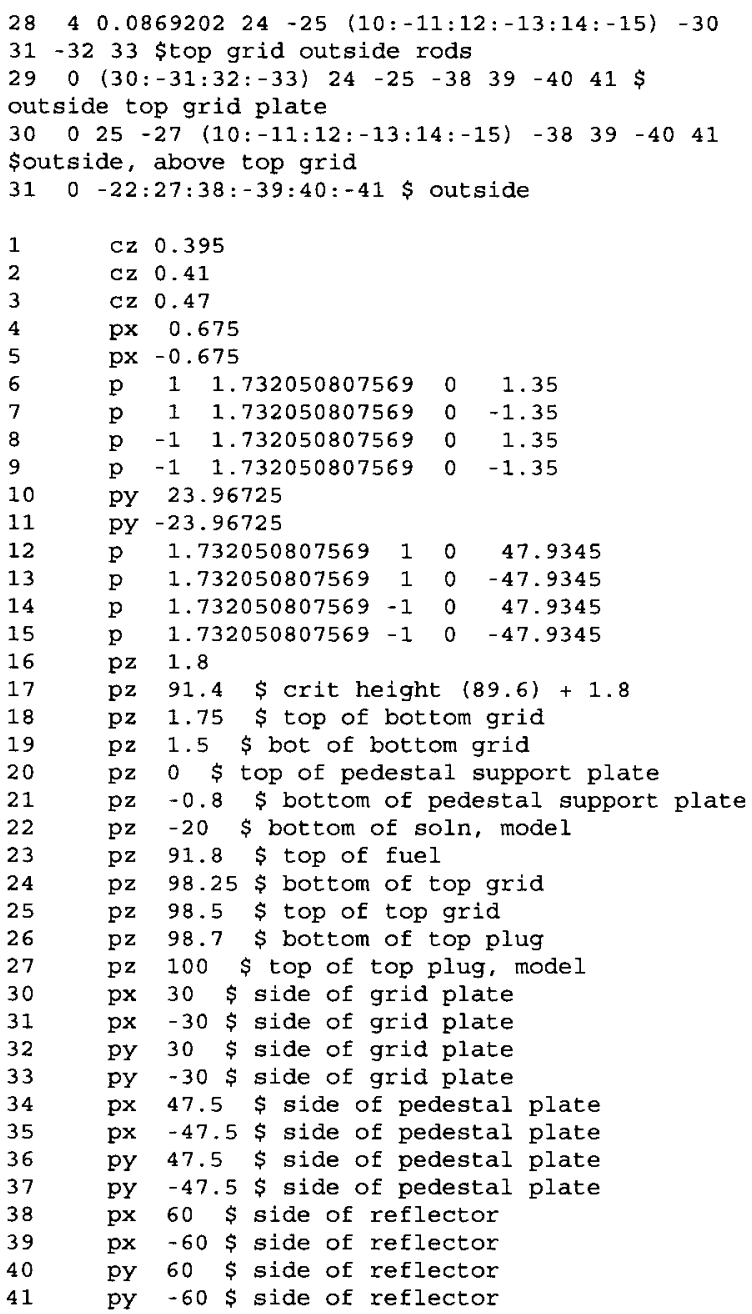

imp: $n \quad 129 r 0$

m1 \$4.38\%-Enriched Uranium Dioxide 0.06954637 $8016 \quad 4.6391 \mathrm{E}-02$

$922347.1318 \mathrm{E}-06$

$922351.1104 \mathrm{E}-03$

$92236 \quad 3.1838 \mathrm{E}-05$

$92238 \quad 2.2006 \mathrm{E}-02$

m2 \$ AGS aluminum alloy 0.0601967

$13027 \quad 5.9569 \mathrm{E}-02$

$12000 \quad 3.1442 \mathrm{E}-04$

$14000 \quad 2.4894 \mathrm{e}-4$

$260006.4052 e-5$

In not available

m3 \$ Water w Gd 0.100103

$\begin{array}{cc}1001 & 6.66997 \mathrm{e}-2 \\ 8016 & 3.33871 \mathrm{e}-2 \\ 7014 & 1.35177 \mathrm{e}-5 \\ 64152 & 4.59556 \mathrm{E}-09 \\ 64154 & 5.00916 \mathrm{E}-08 \\ 64155 & 3.40071 \mathrm{E}-07 \\ 64156 & 4.70356 \mathrm{E}-07 \\ 64157 & 3.59603 \mathrm{E}-07 \\ 64158 & 5.70769 \mathrm{E}-07 \\ 64160 & 5.02295 \mathrm{E}-07 \\ \operatorname{tr} .01 \mathrm{t} & \\ \text { ainless } & \text { Steel Pedestal } 0.0869202 \\ 6000 & 5.8694 \mathrm{E}-02 \\ 4000 & 1.6469 \mathrm{E}-02 \\ 8000 & 8.1061 \mathrm{E}-03 \\ 5055 & 1.7319 \mathrm{E}-03 \\ 4000 & 1.6939 \mathrm{E}-03 \\ 6000 & 1.1883 \mathrm{E}-04 \\ 5031 & 6.1438 \mathrm{E}-05\end{array}$




\section{CHPRC-00455, REV. 0}

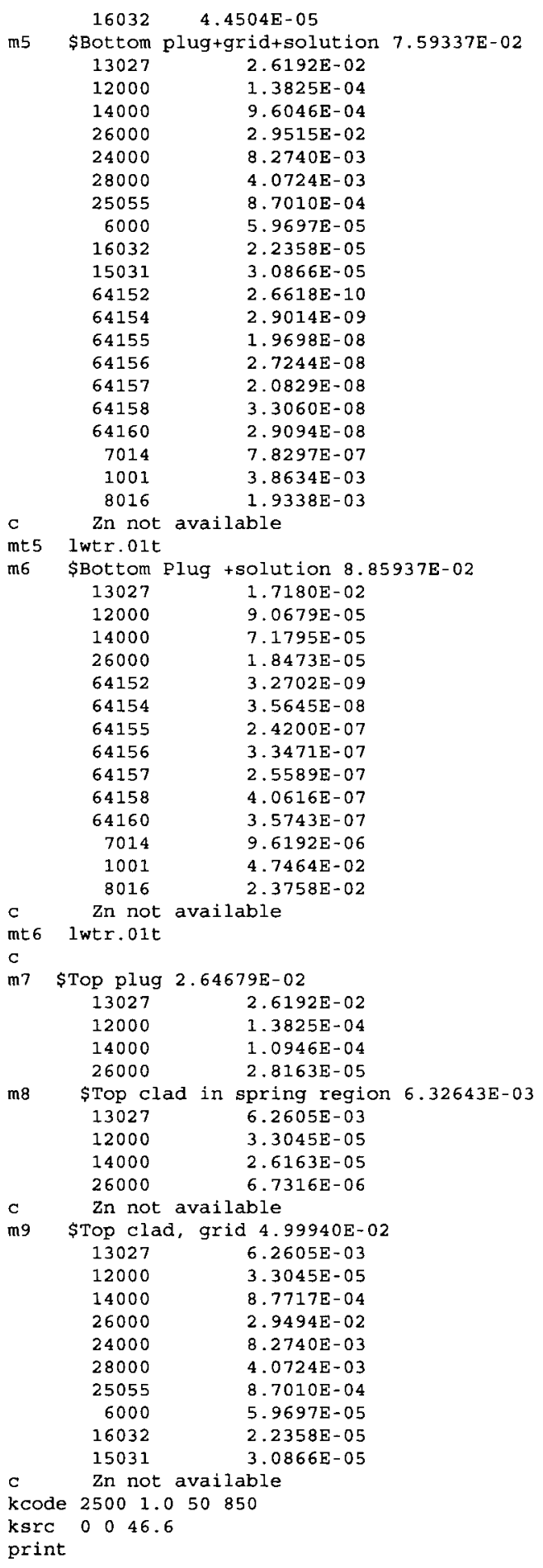

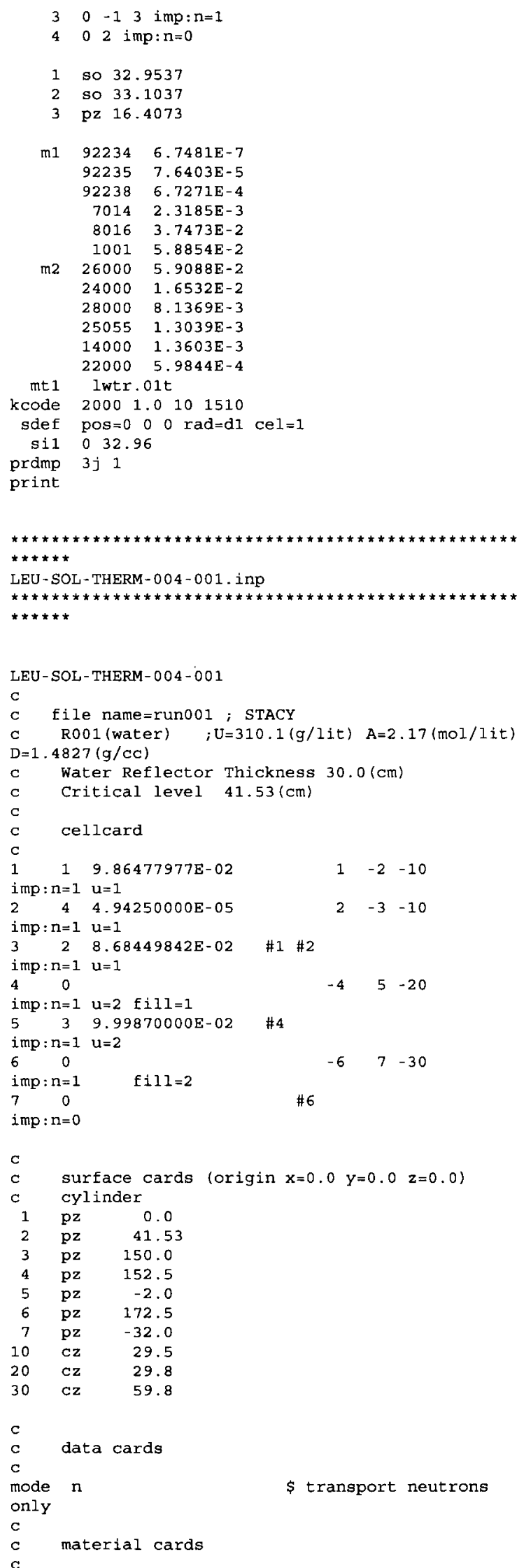


CHPRC-00455, REV. 0

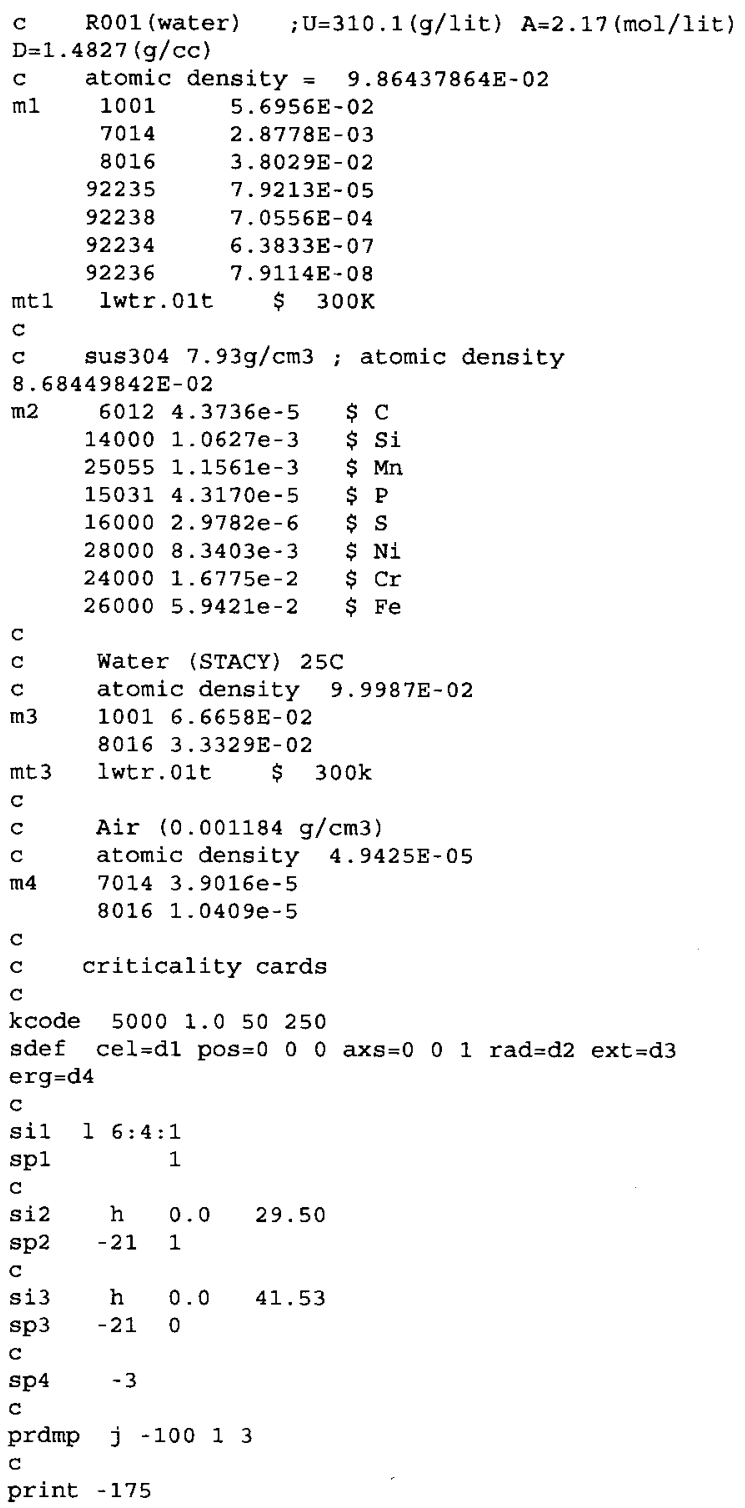

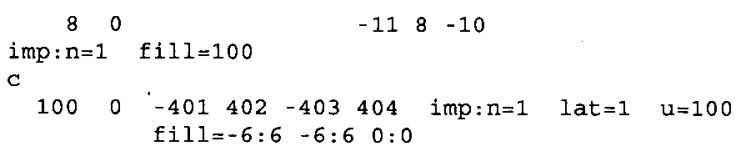




\section{CHPRC-00455, REV. 0}

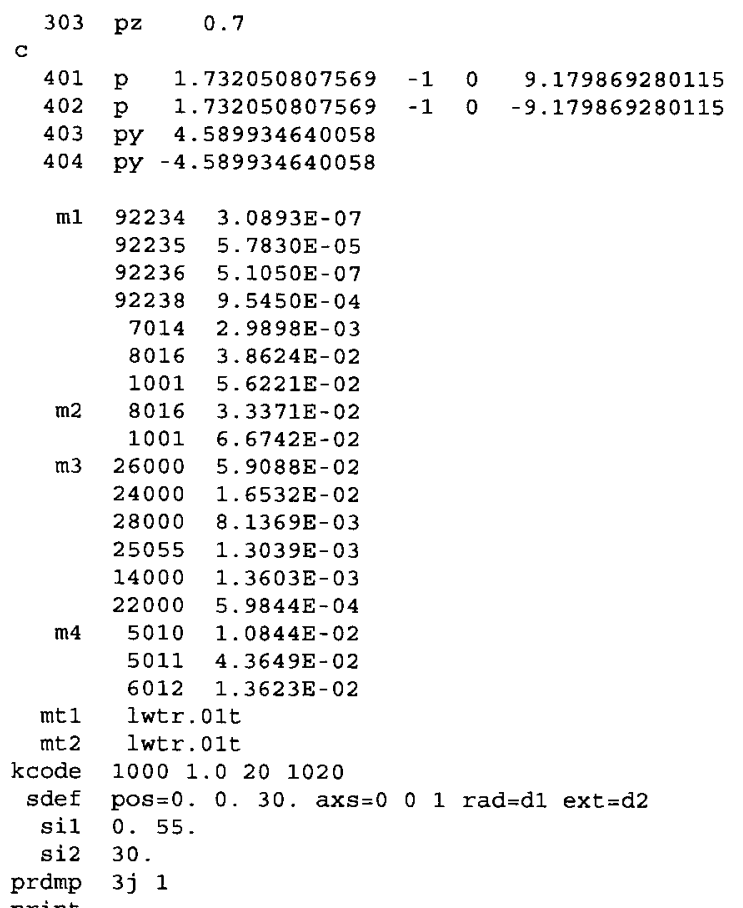

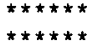

LEU - SOL-THERM-006-001, inp

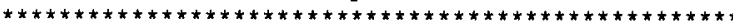

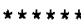

LEU-SOL-THERM-006-001

C leu-sol-therm-006-01

$10 \quad 4:-5: 10$

imp: $\mathrm{n}=0$

$23 \quad 8.701954 \mathrm{E}-02 \quad-4 \quad 5 \quad-10 \quad(3:-6)$

imp: $n=1$

$32 \quad 1.001130 \mathrm{E}-01 \quad-36-9 \quad(2:-7)$

imp : $\mathrm{n}=1$

imp: $n=1$

$53 \quad 8.701954 \mathrm{E}-02 \quad-2 \quad 7 \quad-10 \quad(1:-8)$

$i \mathrm{mp}: \mathrm{n}=1$

$\begin{array}{lllllll}6 & 1 & 9.910035 \mathrm{E}-02 & -1 & 11 & 8 & -201\end{array}$

imp : $\mathrm{n}=1$

imp: $n=1$

$\begin{array}{lllll}-1 & 11 & 201 & -10\end{array}$

imp: $n=1 \quad$ fill $=100$

$100 \quad 0-401 \quad 402-403 \quad 404$ imp: $n=1$ lat $=1 \quad u=100$ fill $=-6: 6 \quad-6: 6 \quad 0: 0$

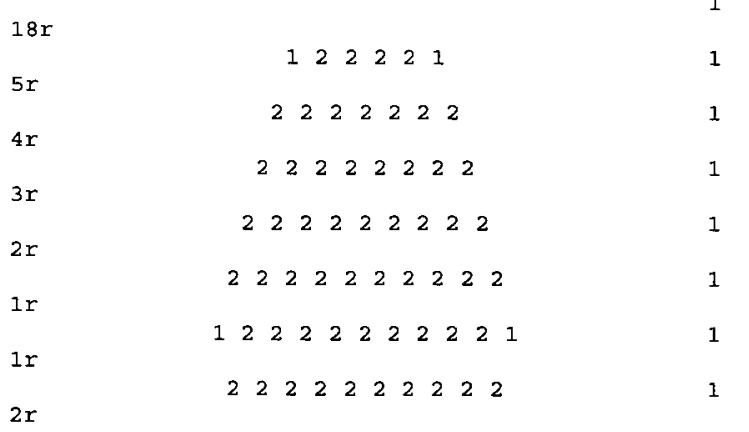

$\begin{array}{lllllllllll}3 r & 2 & 2 & 2 & 2 & 2 & 2 & 2 & 2 & 2 & 1 \\ 4 r & 2 & 2 & 2 & 2 & 2 & 2 & 2 & 2 & 1 \\ 5 r & 2 & 2 & 2 & 2 & 2 & 2 & 2 & 1 \\ 18 r & 1 & 2 & 2 & 2 & 2 & 1 & & 1\end{array}$

18

$1000 \quad 3 \quad 8.701954 \mathrm{E}-02 \quad-200$

imp: $n=1 \quad u=1$

$\begin{array}{lllll}1001 & 1 & 9.910035 \mathrm{E}-02 & 200 & -201\end{array}$

imp: $\mathrm{n}=1 \quad \mathrm{u}=1$

10020

imp : $\mathrm{n}=1 \quad \mathrm{u}=1$

$200019.910035 E-02$

imp: $n=1 \quad u=2$

$200138.701954 E-02$

imp: $n=1 \quad u=2$

$200319.910035 E-02$

imp: $\mathrm{n}=1 \quad \mathrm{u}=2$

20040

$1 \mathrm{mp}: \mathrm{n}=1 \quad \mathrm{u}=2$

c

$300038.701954 \mathrm{E}-02$

imp: $\mathrm{n}=1 \mathrm{u}=3$

$300146.811600 \mathrm{E}-02$

imp: $\mathrm{n}=1 \mathrm{u}=3$

$300238.701954 \mathrm{E}-02$

imp: $n=1 \quad u=3$

$3003 \quad 1 \quad 9.910035 \mathrm{E}-02$

i mp: $n=1 \quad u=3$

$i_{i m p: n=1}^{3004} \begin{array}{llll}3 & 8.701954 \mathrm{E}-02 & 300 & -200\end{array}$

$\begin{array}{llllll}3005 & 1 & 9.910035 E-02 & 301 & 200 & -201\end{array}$

imp: $n=1 \quad u=3$

30060

301

201

imp: $\mathrm{n}=1 \quad \mathrm{u}=3$

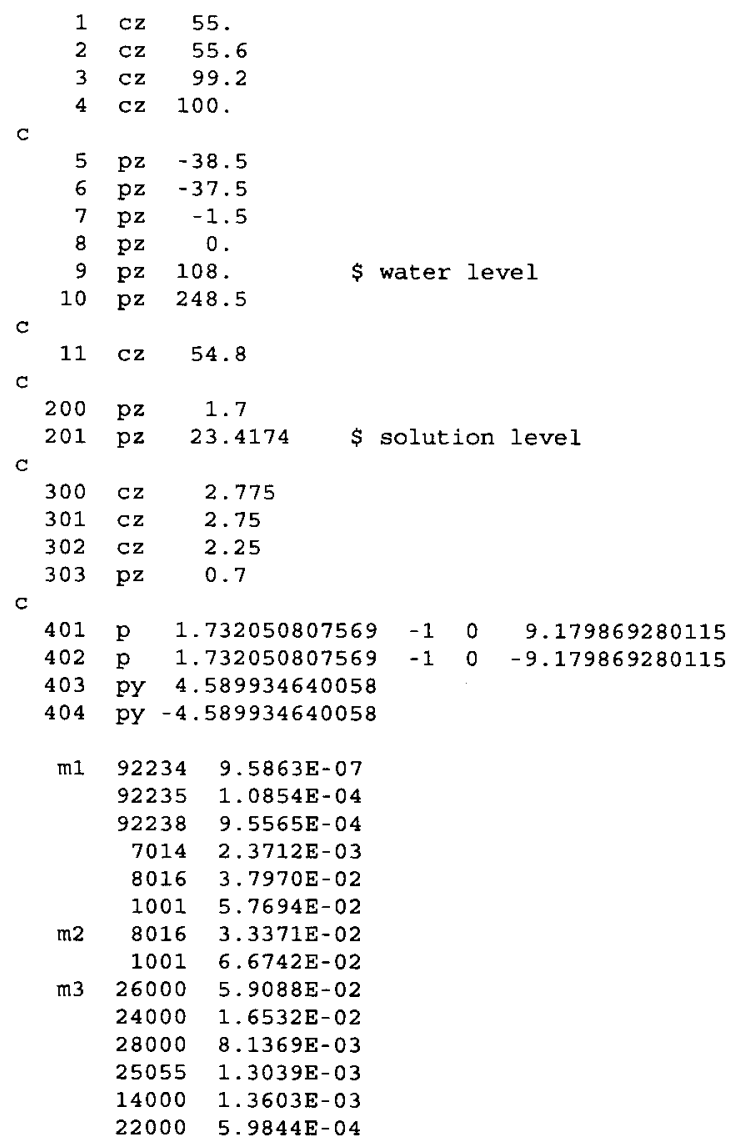




\section{CHPRC-00455, REV. 0}

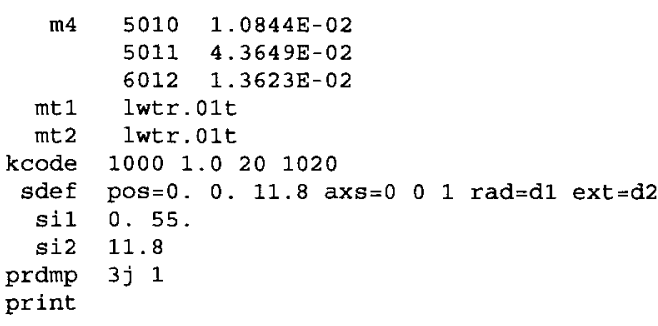

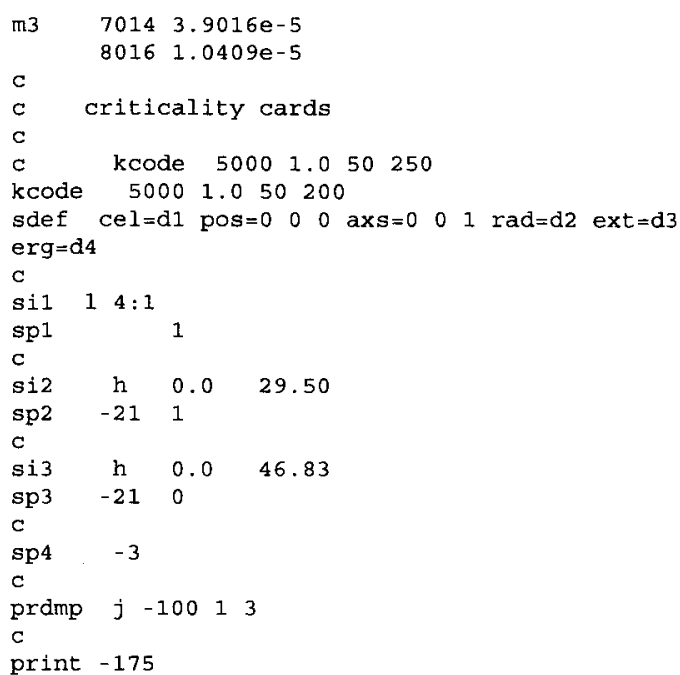




\section{CHPRC-00455, REV. 0}
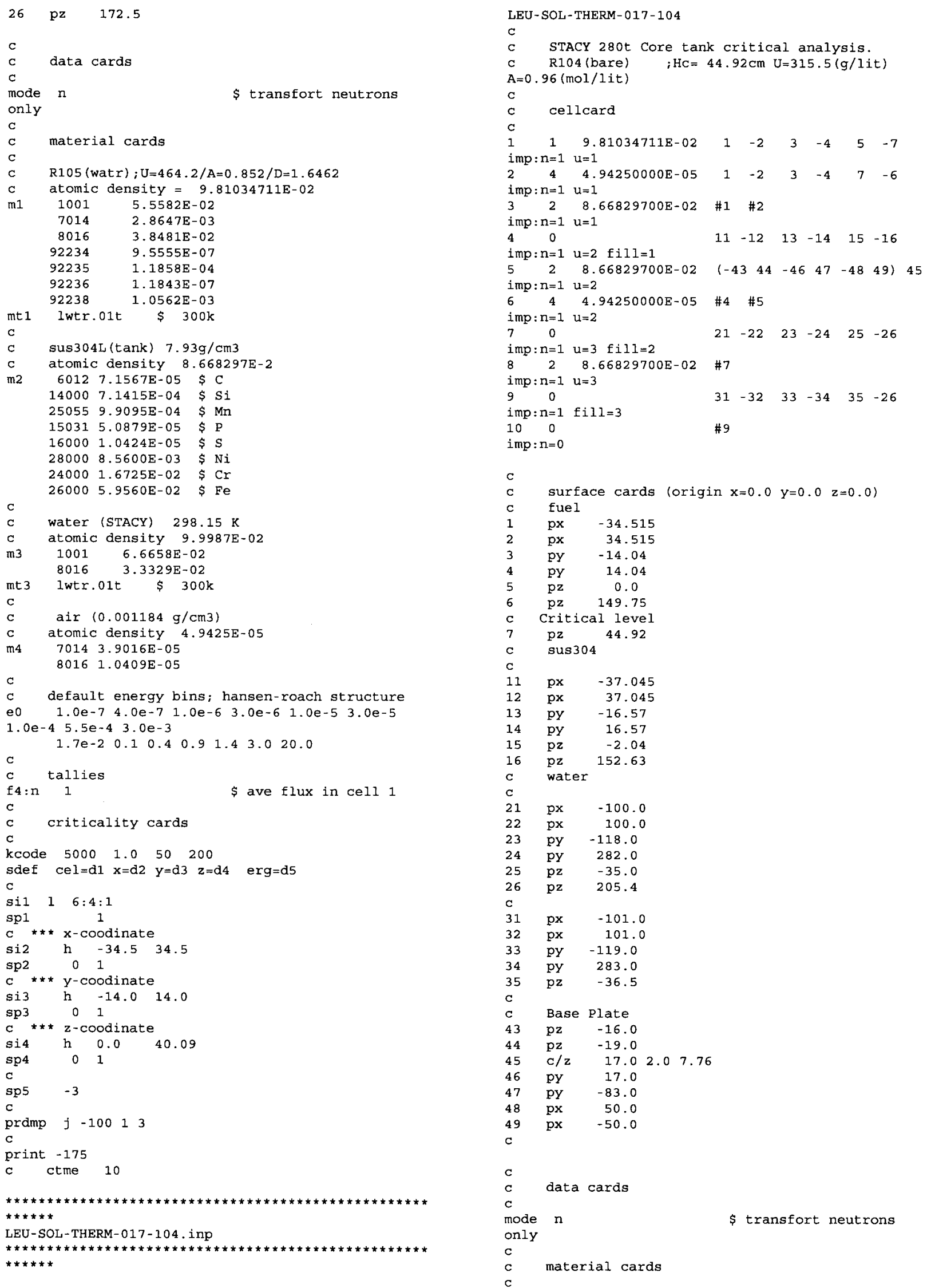


\section{CHPRC-00455, REV. 0}

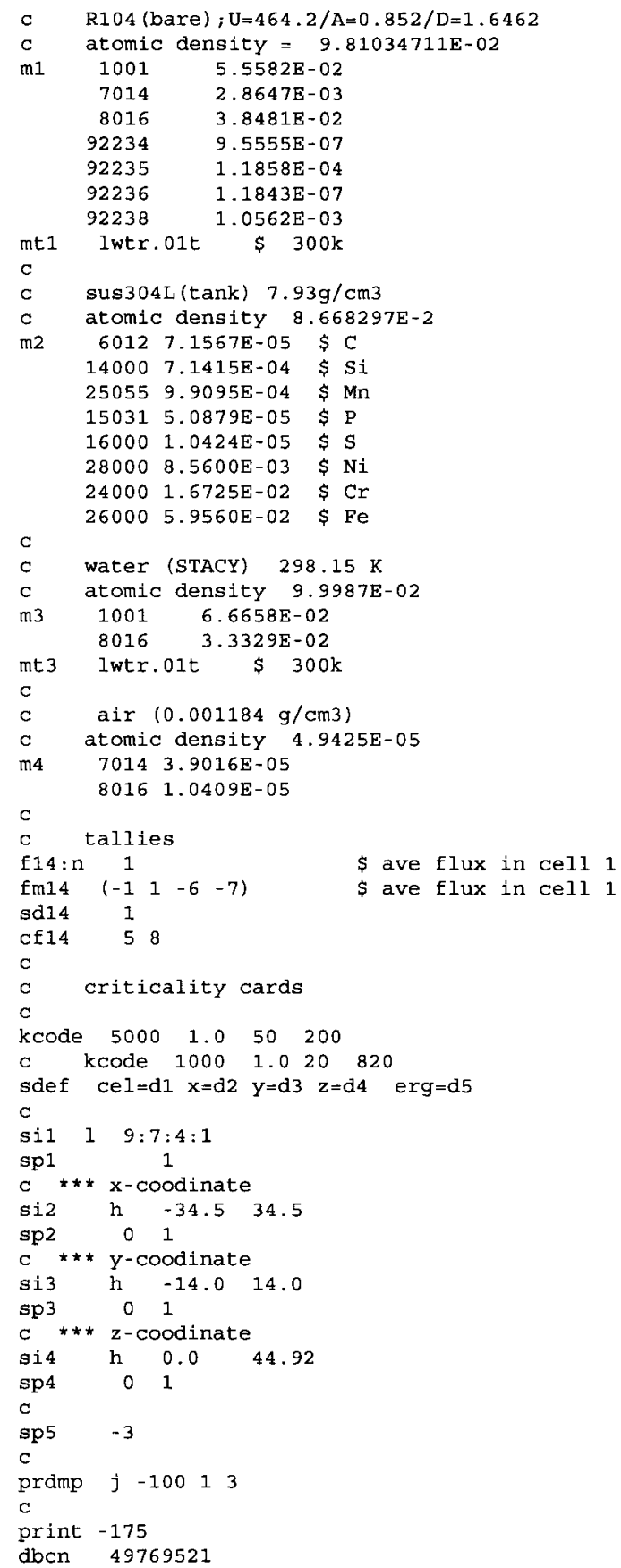


CHPRC-00455, REV. 0

APPENDIX D - USLSTATS OUTPUTS

D-1 
CHPRC-00455, REV. 0

This page is intentionally left blank 
uslstats: a utility to calculate upper subcritical

limits for criticality safety applications

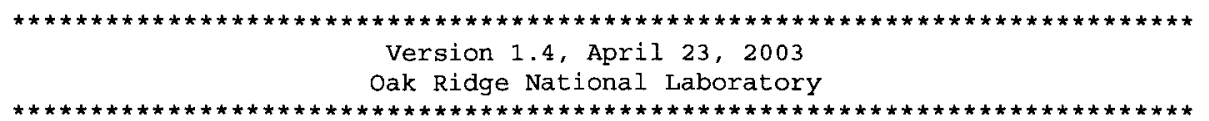

Input to statistical treatment from file:meu-ealf, inp

Title: MEU Benchmarks, EALF

$\begin{array}{llr}\text { Proportion of the population } & =.995 \\ \text { Confidence of fit } & =.950 \\ \text { Confidence on proportion } & =.950 \\ \text { Number of observations } & =167 \\ \text { Minimum value of closed band } & =0.00 \\ \text { Maximum value of closed band } & =0.00 \\ \text { Administrative margin } & =0.05\end{array}$

independent

variable - $\mathbf{x}$

dependent

variable - y

deviation

$9.13000 \mathrm{E}-08$

$1.35000 \mathrm{E}-07$

$9.96000 \mathrm{E}-08$

$1.06000 \mathrm{E}-07$

$9.92000 \mathrm{E}-08$

1. $05000 \mathrm{E}-07$

1. $30000 \mathrm{E}-07$

1. $30000 \mathrm{E}-07$

$1.30000 \mathrm{E}-07$

1.28000E-07

1.27000E-07

$3.51000 \mathrm{E}-07$

3. $52000 \mathrm{E}-07$

$3.51000 \mathrm{E}-07$

3. $53000 \mathrm{E}-07$

2. $36000 \mathrm{E}-07$

2. $82000 \mathrm{E}-07$

$2.43000 \mathrm{E}-07$

2. $90000 \mathrm{E}-07$

3. $34000 \mathrm{E}-07$

3. $26000 \mathrm{E}-07$

2. $52000 \mathrm{E}-07$

2.71000E-07

2. $83000 \mathrm{E}-07$

2. $81000 \mathrm{E}-07$

2. $81000 \mathrm{E}-07$

$2.82000 \mathrm{E}-07$

$2.84000 \mathrm{E}-07$

2. $89000 \mathrm{E}-07$

2. $91000 \mathrm{E}-07$

2. $67000 \mathrm{E}-07$

1. $30000 \mathrm{E}-07$

1. $29000 \mathrm{E}-07$

1. $30000 \mathrm{E}-07$

1.29000E-07

1. $31000 \mathrm{E}-07$

1. $30000 \mathrm{E}-07$

1. $31000 \mathrm{E}-07$

1. 30000 E- 07

1. $31000 \mathrm{E}-07$

1. $30000 \mathrm{E}-07$

1.29000E-07

$1.30000 \mathrm{E}-07$

$1.29000 \mathrm{E}-07$

$1.31000 \mathrm{E}-07$

$1.30000 \mathrm{E}-07$

$1.31000 \mathrm{E}-07$

1. $30000 \mathrm{E}-07$

1. $31000 \mathrm{E}-07$

$1.30000 \mathrm{E}-07$
9. $92200 \mathrm{E}-01$

9. $95600 \mathrm{E}-01$

9. $96900 \mathrm{E}-01$

9. $91400 \mathrm{E}-01$

9. $91700 \mathrm{E}-01$

9. $85900 \mathrm{E}-01$

9. $93200 \mathrm{E}-01$

9. $95000 \mathrm{E}-01$

9. $99700 \mathrm{E}-01$

9. $98600 \mathrm{E}-01$

9. $94500 \mathrm{E}-01$

9. $92500 \mathrm{E}-01$

9. $89200 \mathrm{E}-01$

$9.93700 \mathrm{E}-01$

9. $90000 \mathrm{E}-01$

9. $92300 \mathrm{E}-01$

9. $87200 \mathrm{E}-01$

9. $89300 \mathrm{E}-01$

9. $85300 \mathrm{E}-01$

9. $85500 \mathrm{E}-01$

9. $97100 \mathrm{E}-01$

9. $83000 \mathrm{E}-01$

9. $74600 \mathrm{E}-01$

9. $82000 \mathrm{E}-01$

$9.88100 \mathrm{E}-01$

9. $82500 \mathrm{E}-01$

9. $86900 \mathrm{E}-01$

9. $85600 \mathrm{E}-01$

9. $82000 \mathrm{E}-01$

9. $88800 \mathrm{E}-01$

9.83900E-01

9. $93600 \mathrm{E}-01$

9. $95400 \mathrm{E}-01$

9. $95700 \mathrm{E}-01$

$9.95000 \mathrm{E}-01$

9. $96600 \mathrm{E}-01$

$9.93900 \mathrm{E}-01$

9. $96400 \mathrm{E}-01$

$9.93000 \mathrm{E}-01$

9. $94600 \mathrm{E}-01$

1. $00060 \mathrm{E}+00$

9. $96700 \mathrm{E}-01$

9. $96600 \mathrm{E}-01$

1. $00060 \mathrm{E}+00$

9. $95600 \mathrm{E}-01$

9. $92300 \mathrm{E}-01$

9. $93200 \mathrm{E}-01$

1. $00180 \mathrm{E}+00$

9. $95800 \mathrm{E}-01$

9. $93100 \mathrm{E}-01$

\section{in $y$}

4. $00000 \mathrm{E}-03$

4. $10000 \mathrm{E}-03$

$4.50000 \mathrm{E}-03$

4. $50000 \mathrm{E}-03$

4. $40000 \mathrm{E}-03$

4. $50000 \mathrm{E}-03$

2. 80000 E- 03

2. $80000 \mathrm{E}-03$

2. $80000 \mathrm{E}-03$

2. $80000 \mathrm{E}-03$

2. $80000 \mathrm{E}-03$

3. $80000 \mathrm{E}-03$

4. $00000 \mathrm{E}-03$

3. $90000 \mathrm{E}-03$

3. $80000 \mathrm{E}-03$

$3.80000 E-03$

4. $00000 \mathrm{E}-03$

3. $90000 \mathrm{E}-03$

4. $10000 \mathrm{E}-03$

4. $00000 \mathrm{E}-03$

4. $00000 \mathrm{E}-03$

4. $00000 \mathrm{E}-03$

3. $90000 \mathrm{E}-03$

4. $00000 \mathrm{E}-03$

4. $20000 \mathrm{E}-03$

4. $00000 \mathrm{E}-03$

$4.00000 \mathrm{E}-03$

$4.00000 \mathrm{E}-03$

4. $00000 \mathrm{E}-03$

$4.00000 \mathrm{E}-03$

3. $90000 \mathrm{E}-03$

2. $70000 \mathrm{E}-03$

2.70000E-03

2. $70000 \mathrm{E}-03$

2. $90000 \mathrm{E}-03$

2. $80000 \mathrm{E}-03$

2. $80000 \mathrm{E}-03$

2. $70000 \mathrm{E}-03$

2. $80000 \mathrm{E}-03$

2. $80000 \mathrm{E}-03$

2. $90000 \mathrm{E}-03$

2. $70000 \mathrm{E}-03$

2. $70000 \mathrm{E}-03$

2. $70000 \mathrm{E}-03$

2. $90000 \mathrm{E}-03$

$2.70000 \mathrm{E}-03$

2. $80000 \mathrm{E}-03$

2. 90000 E-03

2. $70000 \mathrm{E}-03$

2. $80000 \mathrm{E}-03$

\begin{abstract}
independent
\end{abstract}
variable - $\mathrm{x}$

$6.41000 \mathrm{E}-07$

5. $96000 \mathrm{E}-07$

5. 57000E-07

4.75000E-07

2. $80000 \mathrm{E}-07$

4. $74000 \mathrm{E}-07$

1. $20000 \mathrm{E}-06$

1. $96000 \mathrm{E}-06$

4. $53000 \mathrm{E}-07$

$6.62000 \mathrm{E}-07$

2. $06000 \mathrm{E}-07$

2. 14000 E-07

2. $23000 \mathrm{E}-07$

2. $21000 \mathrm{E}-07$

2. 17000 E- 07

2. $06000 \mathrm{E}-07$

2. $11000 \mathrm{E}-07$

2. $49000 \mathrm{E}-06$

2. $51000 \mathrm{E}-06$

2. $67000 \mathrm{E}-06$

2. 78000E-06

1. $40000 \mathrm{E}-06$

1. $42000 \mathrm{E}-06$

1. $36000 \mathrm{E}-06$

1. $46000 \mathrm{E}-06$

8. $99000 \mathrm{E}-07$

9. $05000 \mathrm{E}-07$

9.02000E-07

9. $54000 \mathrm{E}-07$

1. $73000 \mathrm{E}-06$

1. $74000 \mathrm{E}-06$

1. $74000 \mathrm{E}-06$

1. $35000 \mathrm{E}-06$

1. $45000 \mathrm{E}-06$

1. $24000 \mathrm{E}-06$

5. $23000 \mathrm{E}-07$

1. $90000 \mathrm{E}-07$

9. $46000 \mathrm{E}-08$

5. $25000 \mathrm{E}-07$

1. $91000 \mathrm{E}-07$

9. $59000 \mathrm{E}-08$

4. $14000 \mathrm{E}-08$

3. $95000 \mathrm{E}-08$

3. $92000 \mathrm{E}-08$

3. $90000 \mathrm{E}-08$

3. $63000 \mathrm{E}-08$

3. $60000 \mathrm{E}-08$

3. $58000 \mathrm{E}-08$

3. $47000 \mathrm{E}-08$

3. $46000 \mathrm{E}-08$ dependent

variable - y

1. $00150 \mathrm{E}+00$

9. $96100 \mathrm{E}-01$

$9.99000 \mathrm{E}-01$

9. $92100 \mathrm{E}-01$

9. $99000 \mathrm{E}-01$

1. $00510 \mathrm{E}+00$

9. $98700 \mathrm{E}-01$

1. $00770 \mathrm{E}+00$

9. $98400 \mathrm{E}-01$

1. $00650 \mathrm{E}+00$

$9.96900 \mathrm{E}-01$

$9.93200 \mathrm{E}-01$

9. $96800 \mathrm{E}-01$

9. $92700 \mathrm{E}-01$

9.95700E-01

$9.96600 \mathrm{E}-01$

9. $94200 \mathrm{E}-01$

9. $95000 \mathrm{E}-01$

9. $94700 \mathrm{E}-01$

$9.94700 \mathrm{E}-01$

9. $94800 \mathrm{E}-01$

9. $95700 \mathrm{E}-01$

9. $96100 \mathrm{E}-01$

9. $95300 \mathrm{E}-01$

$9.93000 \mathrm{E}-01$

9. $94800 \mathrm{E}-01$

$9.97000 \mathrm{E}-01$

9. $92800 \mathrm{E}-01$

9. $94000 \mathrm{E}-01$

9. $94900 \mathrm{E}-01$

$9.93900 \mathrm{E}-01$

$9.95300 \mathrm{E}-01$

9. $94800 \mathrm{E}-01$

9. $95800 \mathrm{E}-01$

9. $95800 \mathrm{E}-01$

$9.90600 \mathrm{E}-01$

$9.93000 \mathrm{E}-01$

9. $99000 \mathrm{E}-01$

$9.95000 \mathrm{E}-01$

9. $91500 \mathrm{E}-01$

9. $88700 \mathrm{E}-01$

9. $95200 \mathrm{E}-01$

9. $94500 \mathrm{E}-01$

9. $98500 \mathrm{E}-01$

9. $92600 E-01$

9. $96000 \mathrm{E}-01$

9. $97100 \mathrm{E}-01$

9.95200E-01

$9.99100 \mathrm{E}-01$

9.97100E-01

deviation

in $y$

3. $30000 E-03$

3. $40000 \mathrm{E}-03$

3. $40000 \mathrm{E}-03$

3. $30000 \mathrm{E}-03$

3. $50000 \mathrm{E}-03$

3. $50000 E-03$

$6.20000 \mathrm{E}-03$

$6.20000 \mathrm{E}-03$

4. $20000 \mathrm{E}-03$

4. $20000 \mathrm{E}-03$

2. $30000 \mathrm{E}-03$

2. $30000 \mathrm{E}-03$

2. $20000 \mathrm{E}-03$

2. $40000 \mathrm{E}-03$

3. $70000 \mathrm{E}-03$

2. $30000 \mathrm{E}-03$

2. $40000 \mathrm{E}-03$ 
CHPRC-00455, REV. 0

\begin{tabular}{|c|c|c|}
\hline $1.31000 \mathrm{E}-07$ & $9.96800 \mathrm{E}-01$ & $2.80000 \mathrm{E}-03$ \\
\hline $1.30000 \mathrm{E}-07$ & $9.95400 E-01$ & $2.80000 E-03$ \\
\hline $1.31000 \mathrm{E}-07$ & $9.99400 \mathrm{E}-01$ & $2.70000 \mathrm{E}-03$ \\
\hline 1. $30000 E-07$ & $9.95800 \mathrm{E}-01$ & $2.80000 \mathrm{E}-03$ \\
\hline $1.29000 \mathrm{E}-07$ & $9.94500 \mathrm{E}-01$ & $2.80000 \mathrm{E}-03$ \\
\hline $1.29000 \mathrm{E}-07$ & $9.98200 \mathrm{E}-01$ & $2.70000 \mathrm{E}-03$ \\
\hline $1.29000 \mathrm{E}-07$ & $9.89400 \mathrm{E}-01$ & $2.70000 \mathrm{E}-03$ \\
\hline $1.29000 \mathrm{E}-07$ & $9.92500 E-01$ & $2.80000 \mathrm{E}-03$ \\
\hline $1.36000 \mathrm{E}-07$ & 1. $00590 \mathrm{E}+00$ & $2.80000 \mathrm{E}-03$ \\
\hline $1.30000 \mathrm{E}-07$ & 1. $00750 \mathrm{E}+00$ & $2.70000 \mathrm{E}-03$ \\
\hline 1. $28000 \mathrm{E}-07$ & $1.00170 \mathrm{E}+00$ & $2.90000 \mathrm{E}-03$ \\
\hline $1.26000 \mathrm{E}-07$ & $9.91800 \mathrm{E}-01$ & $2.70000 \mathrm{E}-03$ \\
\hline $4.87000 \mathrm{E}-07$ & $9.94500 E-01$ & $2.90000 \mathrm{E}-03$ \\
\hline $3.49000 \mathrm{E}-07$ & $9.96600 \mathrm{E}-01$ & $2.80000 \mathrm{E}-03$ \\
\hline $2.68000 E-07$ & $9.96300 \mathrm{E}-01$ & $2.70000 \mathrm{E}-03$ \\
\hline $2.32000 \mathrm{E}-07$ & $9.94600 \mathrm{E}-01$ & $2.90000 \mathrm{E}-03$ \\
\hline $1.41000 \mathrm{E}-07$ & $9.98800 \mathrm{E}-01$ & $2.70000 \mathrm{E}-03$ \\
\hline $1.35000 \mathrm{E}-07$ & $9.98700 \mathrm{E}-01$ & $2.60000 \mathrm{E}-03$ \\
\hline $1.33000 \mathrm{E}-07$ & $9.99300 \mathrm{E}-01$ & $2.80000 \mathrm{E}-03$ \\
\hline 1. $30000 \mathrm{E}-07$ & $9.97800 \mathrm{E}-01$ & $2.80000 \mathrm{E}-03$ \\
\hline $1.27000 \mathrm{E}-07$ & $9.95000 E-01$ & $2.80000 \mathrm{E}-03$ \\
\hline $3.76000 \mathrm{E}-07$ & $9.96600 \mathrm{E}-01$ & $3.40000 \mathrm{E}-03$ \\
\hline $3.61000 \mathrm{E}-07$ & $9.98900 \mathrm{E}-01$ & $3.30000 \mathrm{E}-03$ \\
\hline $3.51000 \mathrm{E}-07$ & $1.00290 \mathrm{E}+00$ & $3.30000 \mathrm{E}-03$ \\
\hline $3.41000 \mathrm{E}-07$ & $9.96000 E-01$ & $3.30000 E-03$ \\
\hline $3.35000 \mathrm{E}-07$ & $9.94200 \mathrm{E}-01$ & $3.40000 E-03$ \\
\hline $3.27000 \mathrm{E}-07$ & $9.96300 \mathrm{E}-01$ & $3.50000 \mathrm{E}-03$ \\
\hline $3.60000 \mathrm{E}-07$ & $1.00330 \mathrm{E}+00$ & $3.40000 \mathrm{E}-03$ \\
\hline $3.49000 \mathrm{E}-07$ & $1.00560 \mathrm{E}+00$ & $3.30000 \mathrm{E}-03$ \\
\hline $3.35000 \mathrm{E}-07$ & $1.00640 \mathrm{E}+00$ & $3.30000 \mathrm{E}-03$ \\
\hline $3.26000 \mathrm{E}-07$ & $9.97800 \mathrm{E}-01$ & $3.40000 \mathrm{E}-03$ \\
\hline $8.21000 E-07$ & $9.97800 \mathrm{E}-01$ & $3.30000 \mathrm{E}-03$ \\
\hline $7.47000 \mathrm{E}-07$ & $9.97800 \mathrm{E}-01$ & $3.30000 \mathrm{E}-03$ \\
\hline $6.88000 E-07$ & $1.00000 \mathrm{E}+00$ & $3.30000 E-03$ \\
\hline
\end{tabular}

4. $21000 \mathrm{E}-08$

$4.10000 \mathrm{E}-08$

3. $98000 \mathrm{E}-08$

$3.90000 \mathrm{E}-08$

3. $84000 \mathrm{E}-08$

$3.79000 \mathrm{E}-08$

3. $75000 \mathrm{E}-08$

4. $12000 \mathrm{E}-08$

4. $12000 \mathrm{E}-08$

4. $15000 \mathrm{E}-08$

4. $86000 \mathrm{E}-08$

$4.89000 \mathrm{E}-08$

4. $93000 \mathrm{E}-08$

4. $94000 \mathrm{E}-08$

4. $99000 \mathrm{E}-08$

4. $28000 \mathrm{E}-08$

4. $15000 \mathrm{E}-08$

4. 01000E-08

3. $93000 \mathrm{E}-08$

3. $87000 \mathrm{E}-08$

5. $22000 \mathrm{E}-08$

4. $96000 \mathrm{E}-08$

4. 57000E-08

4. $44000 \mathrm{E}-08$

4. $31000 \mathrm{E}-08$

4. $22000 \mathrm{E}-08$

4. $15000 \mathrm{E}-08$

5. $23000 \mathrm{E}-08$

$4.99000 \mathrm{E}-08$

4. $57000 \mathrm{E}-08$

$4.45000 \mathrm{E}-08$

4. $31000 \mathrm{E}-08$

4. $24000 \mathrm{E}-08$
9. $99000 \mathrm{E}-01$

9. $98400 \mathrm{E}-01$

$9.97400 \mathrm{E}-01$

$9.99900 \mathrm{E}-01$

1.00020E+00

$9.99900 \mathrm{E}-01$

$9.99500 \mathrm{E}-01$

9. $95800 \mathrm{E}-01$

9. $96500 \mathrm{E}-01$

9.97200E-01

9. $98600 \mathrm{E}-01$

1. $00380 \mathrm{E}+00$

9. $96500 \mathrm{E}-01$

9.98700E-01

9.99500E-01

$9.96600 \mathrm{E}-01$

9. $99400 \mathrm{E}-01$

$9.96800 \mathrm{E}-01$

$9.98800 \mathrm{E}-01$

9. $97400 \mathrm{E}-0 \mathrm{I}$

$00380 \mathrm{E}+00$

1. $00490 \mathrm{E}+00$

$1.00160 \mathrm{E}+00$

1. $00240 \mathrm{E}+00$

1. $00010 \mathrm{E}+00$

1. $00220 \mathrm{E}+00$

1. $00170 \mathrm{E}+00$

1. $00350 \mathrm{E}+00$

$1.00270 \mathrm{E}+00$

$1.00030 \mathrm{E}+00$

$9.99800 \mathrm{E}-01$

1. $00180 \mathrm{E}+00$

1. $00050 \mathrm{E}+00$
$1.00000 \mathrm{E}-03$

$1.10000 \mathrm{E}-03$

$1.10000 \mathrm{E}-03$

1. $20000 \mathrm{E}-03$

$1.10000 \mathrm{E}-03$

1. $20000 \mathrm{E}-03$

1. $20000 \mathrm{E}-03$

$4.20000 \mathrm{E}-03$

$5.10000 \mathrm{E}-03$

$6.40000 \mathrm{E}-03$

3. $80000 \mathrm{E}-03$

3. $90000 \mathrm{E}-03$

4. $20000 E-03$

4. $20000 \mathrm{E}-03$

$4.80000 \mathrm{E}-03$

$1.30000 \mathrm{E}-03$

$1.20000 \mathrm{E}-03$

1. $30000 \mathrm{E}-03$

1. $30000 \mathrm{E}-03$

1. $30000 \mathrm{E}-03$

$1.60000 \mathrm{E}-03$

1. $60000 \mathrm{E}-03$

$1.60000 \mathrm{E}-03$

1. $60000 \mathrm{E}-03$

1. $60000 \mathrm{E}-03$

1. $70000 \mathrm{E}-03$

1. $70000 \mathrm{E}-03$

$1.60000 \mathrm{E}-03$

$1.60000 \mathrm{E}-03$

1. $70000 \mathrm{E}-03$

$1.60000 \mathrm{E}-03$

$1.70000 \mathrm{E}-03$

1. $70000 \mathrm{E}-03$

chi $=17.8802$ (upper bound $=9.49$ ). The data tests NOT normal

Output from statistical treatment

MEU Benchmarks, EALF

Number of data points (n)

Linear regression, $\mathrm{k}(\mathrm{X})$

Confidence on fit ( 1 -gamma) [input]

Confidence on proportion (alpha) [input]

Proportion of population falling above

lower tolerance interval (rho) [input]

Minimum value of $x$

Maximum value of $x$

Average value of $x$

Average value of $k$

Minimum value of $k$

Variance of fit, $s(k, X)^{\wedge} 2$

within variance, $s(w) \wedge 2$

Pooled variance, $s(p)^{\wedge} 2$

Pooled std. deviation, $s(p)$

C (alpha, rho)*s (p)

student-t @ (n-2,1-gamma)

Confidence band width, w

Minimum margin of subcriticality, $C^{*} s(p)-W$
167

$0.9961+(-4.4477 \mathrm{E}+02) * \mathrm{X}$

$95.0 \%$

$95.0 \%$

$99.5 \%$

$3.4600 \mathrm{E}-08$

2. $7800 \mathrm{E}-06$

$3.6003 \mathrm{E}-07$

0.99596

0.97460

$2.7003 \mathrm{E}-05$

1. $1262 \mathrm{E}-05$

$3.8265 \mathrm{E}-05$

$6.1859 \mathrm{E}-03$

$2.8223 \mathrm{E}-02$

$1.64500 \mathrm{E}+00$

$1.0833 \mathrm{E}-02$

$1.7391 \mathrm{E}-02$

Upper subcritical limits: $(3.46000 \mathrm{E}-08<=\mathrm{X}<=2.78000 \mathrm{E}-06)$

USL Method 1 (Confidence Band with

Administrative Margin)

USL1 $=0.9353+(-4.4477 \mathrm{E}+02) * \mathrm{X}$

USL Method 2 (Single-Sided Uniform

width Closed Interval Approach) USL2 $=0.9679+(-4.4477 \mathrm{E}+02) * \mathrm{X}$

USLs Evaluated Over Range of Parameter X:

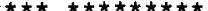




\section{CHPRC-00455, REV. 0}

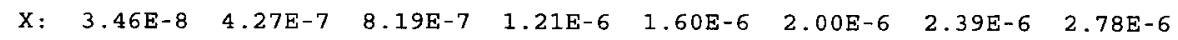

$\begin{array}{lllllllll}\text { USL-1 : } & 0.9353 & 0.9351 & 0.9349 & 0.9348 & 0.9346 & 0.9344 & 0.9342 & 0.9341\end{array}$

$\begin{array}{lllllllll}\text { USL-2: } & 0.9679 & 0.9677 & 0.9675 & 0.9674 & 0.9672 & 0.9670 & 0.9668 & 0.9667\end{array}$

Thus spake USLSTATS Finis.

Plot file written to: meu-ealf.plt 


\section{CHPRC-00455, REV. 0}

uslstats: a utility to calculate upper subcritical

limits for criticality safety applications

Version 1.4, April 23, 2003

Oak Ridge National Laboratory

Input to statistical treatment from file:meu-h_x-60.inp

Title: MEU Benchmark.s, $h / x$

Proportion of the population $=.995$

Confidence of fit $\quad=.950$

Confidence on proportion $=.950$

Number of observations $=60$

Minimum value of closed band $=0.00$

Maximum value of closed band $=0.00$

Administrative margin $\quad=0.05$

\begin{tabular}{|c|c|c|c|c|c|}
\hline independent & dependent & deviation & independent & dependent & deviation \\
\hline variable - $x$ & variable - y & in $y$ & variable - $x$ & variable - y & in $y$ \\
\hline $3.99430 \mathrm{E}+01$ & $9.95000 \mathrm{E}-01$ & $3.50000 \mathrm{E}-03$ & $8.96000 \mathrm{E}+02$ & $9.99900 \mathrm{E}-01$ & 1. $20000 \mathrm{E}-03$ \\
\hline $3.99430 \mathrm{E}+01$ & $9.94700 \mathrm{E}-01$ & $3.40000 E-03$ & $9.42000 \mathrm{E}+02$ & $1.00020 \mathrm{E}+00$ & $1.10000 E-03$ \\
\hline $3.99430 \mathrm{E}+01$ & $9.94700 \mathrm{E}-01$ & $3.50000 \mathrm{E}-03$ & $9.83000 \mathrm{E}+02$ & $9.99900 \mathrm{E}-01$ & $1.20000 \mathrm{E}-03$ \\
\hline $3.99430 \mathrm{E}+01$ & $9.94800 \mathrm{E}-01$ & $3.50000 \mathrm{E}-03$ & $1.01800 \mathrm{E}+03$ & $9.99500 \mathrm{E}-01$ & 1. $20000 \mathrm{E}-03$ \\
\hline $4.99471 E+01$ & $9.95700 \mathrm{E}-01$ & $4.20000 E-03$ & $9.72177 E+02$ & $9.95800 \mathrm{E}-01$ & $4.20000 E-03$ \\
\hline $4.99471 E+01$ & $9.96100 \mathrm{E}-01$ & $4.30000 E-03$ & $9.72177 \mathrm{E}+02$ & $9.96500 \mathrm{E}-01$ & $5.10000 \mathrm{E}-03$ \\
\hline $4.99471 \mathrm{E}+01$ & $9.95300 \mathrm{E}-01$ & $4.20000 \mathrm{E}-03$ & $9.72177 \mathrm{E}+02$ & $9.97200 \mathrm{E}-01$ & $6.40000 \mathrm{E}-03$ \\
\hline $4.99471 E+01$ & $9.93000 \mathrm{E}-01$ & $4.20000 E-03$ & $5.31546 \mathrm{E}+02$ & $9.98600 \mathrm{E}-01$ & $3.80000 E-03$ \\
\hline $5.96772 \mathrm{E}+01$ & $9.94800 \mathrm{E}-01$ & $3.80000 E-03$ & $5.31546 E+02$ & $1.00380 \mathrm{E}+00$ & $3.90000 E-03$ \\
\hline $5.96772 \mathrm{E}+01$ & $9.97000 \mathrm{E}-01$ & $3.80000 \mathrm{E}-03$ & $5.31546 \mathrm{E}+02$ & $9.96500 \mathrm{E}-01$ & $4.20000 E-03$ \\
\hline $5.96772 E+01$ & $9.92800 \mathrm{E}-01$ & $3.80000 E-03$ & $5.31546 E+02$ & $9.98700 \mathrm{E}-01$ & $4.20000 E-03$ \\
\hline $5.96772 E+01$ & $9.94000 \mathrm{E}-01$ & $3.80000 E-03$ & $5.31546 \mathrm{E}+02$ & $9.99500 \mathrm{E}-01$ & $4.80000 \mathrm{E}-03$ \\
\hline $5.96772 \mathrm{E}+01$ & $9.94900 \mathrm{E}-01$ & $3.70000 E-03$ & $7.09245 E+02$ & $9.96600 \mathrm{E}-01$ & $1.30000 \mathrm{E}-03$ \\
\hline $5.96772 \mathrm{E}+01$ & $9.93900 \mathrm{E}-01$ & $3.70000 E-03$ & $7.69975 \mathrm{E}+02$ & $9.99400 \mathrm{E}-01$ & $1.20000 E-03$ \\
\hline $5.96772 \mathrm{E}+01$ & $9.95300 \mathrm{E}-01$ & $3.70000 \mathrm{E}-03$ & $8.42178 \mathrm{E}+02$ & $9.96800 E-01$ & $1.30000 \mathrm{E}-03$ \\
\hline $5.96772 \mathrm{E}+01$ & $9.94800 E-01$ & $3.70000 \mathrm{E}-03$ & $8.96048 \mathrm{E}+02$ & $9.98800 E-01$ & $1.30000 \mathrm{E}-03$ \\
\hline $5.96772 E+01$ & $9.95800 \mathrm{E}-01$ & $3.60000 E-03$ & $9.42242 E+02$ & $9.97400 \mathrm{E}-01$ & 1. $30000 E-03$ \\
\hline $5.96772 E+01$ & $9.95800 \mathrm{E}-01$ & $3.10000 \mathrm{E}-03$ & $4.68730 \mathrm{E}+02$ & $1.00380 \mathrm{E}+00$ & $1.60000 \mathrm{E}-03$ \\
\hline $7.70310 \mathrm{E}+02$ & $9.95200 \mathrm{E}-01$ & $3.90000 \mathrm{E}-03$ & $5.14152 \mathrm{E}+02$ & $1.00490 \mathrm{E}+00$ & $1.60000 \mathrm{E}-03$ \\
\hline $8.77596 \mathrm{E}+02$ & $9.94500 \mathrm{E}-01$ & $4.20000 E-03$ & $5.08428 \mathrm{E}+02$ & $1.00160 \mathrm{E}+00$ & $1.60000 \mathrm{E}-03$ \\
\hline $8.97006 \mathrm{E}+02$ & $9.98500 \mathrm{E}-01$ & $4.20000 E-03$ & $6.50214 E+02$ & $1.00240 \mathrm{E}+00$ & $1.60000 E-03$ \\
\hline $9.13248 \mathrm{E}+02$ & $9.92600 \mathrm{E}-01$ & $4.20000 \mathrm{E}-03$ & $6.99137 \mathrm{E}+02$ & $1.00010 E+00$ & $1.60000 \mathrm{E}-03$ \\
\hline $1.17338 \mathrm{E}+03$ & $9.96000 \mathrm{E}-01$ & $4.80000 E-03$ & $7.38928 \mathrm{E}+02$ & $1.00220 \mathrm{E}+00$ & $1.70000 \mathrm{E}-03$ \\
\hline $1.21310 \mathrm{E}+03$ & $9.97100 \mathrm{E}-01$ & $4.90000 E-03$ & $7.71792 \mathrm{E}+02$ & $1.00170 \mathrm{E}+00$ & $1.70000 \mathrm{E}-03$ \\
\hline $1.23980 \mathrm{E}+03$ & $9.95200 \mathrm{E}-01$ & $4.90000 \mathrm{E}-03$ & $4.68730 \mathrm{E}+02$ & $1.00350 E+00$ & $1.60000 \mathrm{E}-03$ \\
\hline $1.41164 \mathrm{E}+03$ & $9.99100 E-01$ & $5.20000 E-03$ & $5.10846 \mathrm{E}+02$ & $1.00270 \mathrm{E}+00$ & $1.60000 \mathrm{E}-03$ \\
\hline $1.43751 \mathrm{E}+03$ & $9.97100 \mathrm{E}-01$ & $5.20000 \mathrm{E}-03$ & $6.10947 \mathrm{E}+02$ & $1.00030 \mathrm{E}+00$ & $1.70000 \mathrm{E}-03$ \\
\hline $7.19000 \mathrm{E}+02$ & $9.99000 E-01$ & $1.00000 E-03$ & $6.50082 E+02$ & $9.99800 \mathrm{E}-01$ & $1.60000 \mathrm{E}-03$ \\
\hline $7.71000 \mathrm{E}+02$ & $9.98400 \mathrm{E}-01$ & $1.10000 \mathrm{E}-03$ & $6.99208 \mathrm{E}+02$ & $1.00180 \mathrm{E}+00$ & $1.70000 \mathrm{E}-03$ \\
\hline $842000 F+02$ & $997400 E-01$ & $110000 F-03$ & $728995 \mathrm{E}+02$ & $100050 E+00$ & $170000 E-03$ \\
\hline
\end{tabular}

chi $=3.3333$ (upper bound $=9.49$ ). The data tests normal.

output from statistical treatment

MEU Benchmarks, $h / x$

Number of data points ( $n$ )

60

Linear regression, $\mathrm{k}(\mathrm{x})$

Confidence on fit (1-gamma) [input]

$0.9964+(2.5141 \mathrm{E}-06) * \mathrm{X}$

Proportion of population falling [input]

lower tolerance interval (rho) [input]

Minimum value of $x$

Maximum value of $x$

Average value of $x$

Average value of $k$

Minimum value of $k$

$99.5 \%$

$3.9943 E+01$

$1.4375 \mathrm{E}+03$

$5.8192 \mathrm{E}+02$

0.99782

0.99260 


\section{CHPRC-00455, REV. 0}

Variance of fit, $s(k, x)^{\wedge} 2$

within variance, $s(w) \wedge 2$

8.4667E-06

1.1212E-05

Pooled variance, $s(p)^{\wedge} 2$

1. $9679 \mathrm{E}-05$

Pooled std. deviation, $s(p)$

4. $4361 \mathrm{E}-03$

C (alpha, rho) $\star_{s}(\mathrm{p})$

$1.6700 \mathrm{E}-02$

student-t@(n-2,1-gamma)

1. $67230 \mathrm{E}+00$

Confidence band width, w

7. $7543 \mathrm{E}-03$

Minimum margin of subcriticality, $C^{\star S}(\mathrm{p})-\mathrm{W} \quad 8.9460 \mathrm{E}-03$

Upper subcritical limits: ( $39.943 \quad<=x<=1437.5)$

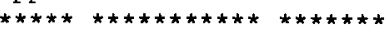

USL Method 1 (Confidence Band with

Administrative Margin)

USL1 $=0.9386+(2.5141 \mathrm{E}-06) * \mathrm{X}$

USL Method 2 (Single-sided Uniform

Width Closed Interval Approach) USL2 $=0.9797+(2.5141 \mathrm{E}-06) * \mathrm{X}$

USLs Evaluated Over Range of Parameter X:

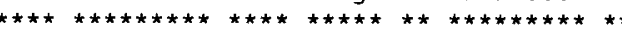

$\mathrm{X}: \quad 3.99 \mathrm{E}+1 \quad 2.40 \mathrm{E}+2 \quad 4.39 \mathrm{E}+2 \quad 6.39 \mathrm{E}+2 \quad 8.39 \mathrm{E}+2 \quad 1.04 \mathrm{E}+3 \quad 1.24 \mathrm{E}+3 \quad 1.44 \mathrm{E}+3$

(1)

$\begin{array}{lllllllll}\text { USL-1: } & 0.9387 & 0.9392 & 0.9397 & 0.9402 & 0.9407 & 0.9412 & 0.9417 & 0.9422\end{array}$

USL-2: $\quad \begin{array}{llllllll}0.9798 & 0.9803 & 0.9808 & 0.9813 & 0.9818 & 0.9823 & 0.9828 & 0.9833\end{array}$

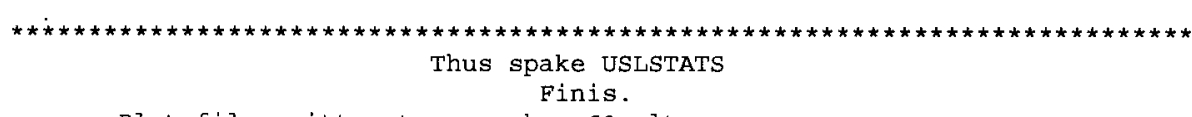

Plot file written to: meu-h_x-60.plt 
CHPRC-00455, REV. 0

uslstats: a utility to calculate upper subcritical

limits for criticality safety applications

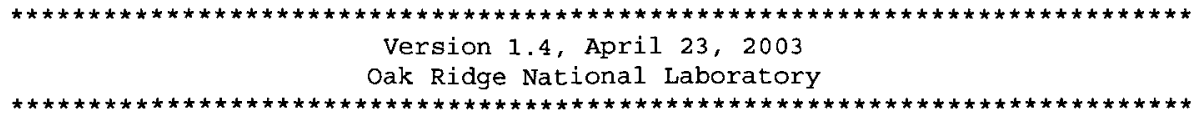

Input to statistical treatment from file:meu-U235_U.inp

Title: MEU Benchmarks, U-235/U

$\begin{array}{llr}\text { Proportion of the population } & =.995 \\ \text { Confidence of fit } & =.950 \\ \text { Confidence on proportion } & =.950 \\ \text { Number of observations } & =167 \\ \text { Minimum value of closed band } & =0.00 \\ \text { Maximum value of closed band } & =0.00 \\ \text { Administrative margin } & =0.05\end{array}$

\begin{tabular}{|c|c|c|c|c|c|}
\hline independent & dependent & deviation & independent & dependent & deviation \\
\hline variable - $\mathbf{x}$ & variable - y & in $y$ & variable - $x$ & variable - y & in $y$ \\
\hline $1.70000 \mathrm{E}-01$ & $9.92200 \mathrm{E}-01$ & $4.00000 E-03$ & $4.31000 \mathrm{E}-02$ & I. $00150 \mathrm{E}+00$ & $3.30000 E-03$ \\
\hline $1.70000 \mathrm{E}-01$ & $9.95600 \mathrm{E}-01$ & $4.10000 E-03$ & $4.31000 \mathrm{E}-02$ & $9.96100 \mathrm{E}-01$ & $3.40000 \mathrm{E}-03$ \\
\hline $1.70000 \mathrm{E}-01$ & $9.96900 \mathrm{E}-01$ & $4.50000 \mathrm{E}-03$ & $4.31000 \mathrm{E}-02$ & $9.99000 \mathrm{E}-01$ & $3.40000 \mathrm{E}-03$ \\
\hline $1.70000 E-01$ & $9.91400 \mathrm{E}-01$ & $4.50000 \mathrm{E}-03$ & $4.31000 \mathrm{E}-02$ & $9.92100 \mathrm{E}-01$ & $3.30000 \mathrm{E}-03$ \\
\hline $1.70000 \mathrm{E}-01$ & $9.91700 \mathrm{E}-01$ & $4.40000 \mathrm{E}-03$ & $4.92000 \mathrm{E}-02$ & $9.99000 \mathrm{E}-01$ & $3.50000 \mathrm{E}-03$ \\
\hline $1.70000 \mathrm{E}-01$ & $9.85900 \mathrm{E}-01$ & $4.50000 \mathrm{E}-03$ & $4.92000 \mathrm{E}-02$ & $1.00510 \mathrm{E}+00$ & $3.50000 \mathrm{E}-03$ \\
\hline $4.31000 \mathrm{E}-02$ & $9.93200 \mathrm{E}-01$ & $2.80000 E-03$ & $4.92000 \mathrm{E}-02$ & $9.98700 \mathrm{E}-01$ & $6.20000 \mathrm{E}-03$ \\
\hline $4.31000 \mathrm{E}-02$ & $9.95000 \mathrm{E}-01$ & $2.80000 \mathrm{E}-03$ & $4.92000 \mathrm{E}-02$ & $1.00770 \mathrm{E}+00$ & $6.20000 \mathrm{E}-03$ \\
\hline $4.31000 E-02$ & $9.99700 \mathrm{E}-01$ & $2.80000 \mathrm{E}-03$ & $4.92000 \mathrm{E}-02$ & $9.98400 \mathrm{E}-01$ & $4.20000 \mathrm{E}-03$ \\
\hline $4.31000 \mathrm{E}-02$ & $9.98600 \mathrm{E}-01$ & $2.80000 \mathrm{E}-03$ & $4.92000 \mathrm{E}-02$ & $1.00650 \mathrm{E}+00$ & $4.20000 \mathrm{E}-03$ \\
\hline $4.31000 \mathrm{E}-02$ & $9.94500 E-01$ & $2.80000 E-03$ & $2.35000 \mathrm{E}-02$ & $9.96900 \mathrm{E}-01$ & $2.30000 \mathrm{E}-03$ \\
\hline $4.31000 \mathrm{E}-02$ & $9.92500 E-01$ & $3.80000 E-03$ & $2.35000 \mathrm{E}-02$ & $9.93200 \mathrm{E}-01$ & $2.30000 \mathrm{E}-03$ \\
\hline $4.31000 \mathrm{E}-02$ & $9.89200 \mathrm{E}-01$ & $4.00000 E-03$ & $2.35000 \mathrm{E}-02$ & $9.96800 \mathrm{E}-01$ & $2.20000 E-03$ \\
\hline $4.31000 E-02$ & $9.93700 E-01$ & $3.90000 E-03$ & $2.35000 \mathrm{E}-02$ & $9.92700 \mathrm{E}-01$ & $2.40000 \mathrm{E}-03$ \\
\hline $4.31000 \mathrm{E}-02$ & $9.90000 E-01$ & $3.80000 E-03$ & $2.35000 \mathrm{E}-02$ & $9.95700 \mathrm{E}-01$ & $3.70000 \mathrm{E}-03$ \\
\hline $4.31000 \mathrm{E}-02$ & $9.92300 \mathrm{E}-01$ & $3.80000 \mathrm{E}-03$ & $2.35000 \mathrm{E}-02$ & $9.96600 \mathrm{E}-01$ & $2.30000 E-03$ \\
\hline $4.31000 \mathrm{E}-02$ & $9.87200 \mathrm{E}-01$ & $4.00000 E-03$ & $2.35000 \mathrm{E}-02$ & $9.94200 \mathrm{E}-01$ & $2.40000 \mathrm{E}-03$ \\
\hline $4.31000 \mathrm{E}-02$ & $9.89300 \mathrm{E}-01$ & $3.90000 \mathrm{E}-03$ & $5.00000 E-02$ & $9.95000 \mathrm{E}-01$ & $3.50000 \mathrm{E}-03$ \\
\hline $4.31000 \mathrm{E}-02$ & $9.85300 \mathrm{E}-01$ & $4.10000 \mathrm{E}-03$ & $5.00000 E-02$ & $9.94700 \mathrm{E}-01$ & $3.40000 \mathrm{E}-03$ \\
\hline 4. $31000 \mathrm{E}-02$ & $9.85500 \mathrm{E}-01$ & $4.00000 E-03$ & $5.00000 E-02$ & $9.94700 \mathrm{E}-01$ & $3.50000 \mathrm{E}-03$ \\
\hline $4.31000 \mathrm{E}-02$ & $9.97100 \mathrm{E}-01$ & $4.00000 \mathrm{E}-03$ & $5.00000 \mathrm{E}-02$ & $9.94800 \mathrm{E}-01$ & $3.50000 \mathrm{E}-03$ \\
\hline $4.31000 \mathrm{E}-02$ & $9.83000 \mathrm{E}-01$ & $4.00000 \mathrm{E}-03$ & $5.00000 \mathrm{E}-02$ & $9.95700 \mathrm{E}-01$ & $4.20000 \mathrm{E}-03$ \\
\hline $4.31000 E-02$ & $9.74600 \mathrm{E}-01$ & $3.90000 \mathrm{E}-03$ & $5.00000 \mathrm{E}-02$ & $9.96100 \mathrm{E}-01$ & $4.30000 E-03$ \\
\hline $4.31000 \mathrm{E}-02$ & $9.82000 E-01$ & $4.00000 E-03$ & $5.00000 \mathrm{E}-02$ & $9.95300 \mathrm{E}-01$ & $4.20000 \mathrm{E}-03$ \\
\hline $4.31000 \mathrm{E}-02$ & $9.88100 E-01$ & $4.20000 \mathrm{E}-03$ & $5.00000 \mathrm{E}-02$ & $9.93000 E-01$ & $4.20000 \mathrm{E}-03$ \\
\hline $4.31000 \mathrm{E}-02$ & $9.82500 \mathrm{E}-01$ & $4.00000 \mathrm{E}-03$ & $5.00000 \mathrm{E}-02$ & $9.94800 \mathrm{E}-01$ & $3.80000 \mathrm{E}-03$ \\
\hline $4.31000 \mathrm{E}-02$ & $9.86900 \mathrm{E}-01$ & $4.00000 E-03$ & $5.00000 \mathrm{E}-02$ & $9.97000 \mathrm{E}-01$ & $3.80000 \mathrm{E}-03$ \\
\hline $4.31000 E-02$ & $9.85600 \mathrm{E}-01$ & $4.00000 E-03$ & $5.00000 E-02$ & $9.92800 \mathrm{E}-01$ & $3.80000 \mathrm{E}-03$ \\
\hline $4.31000 \mathrm{E}-02$ & $9.82000 \mathrm{E}-01$ & $4.00000 \mathrm{E}-03$ & $5.00000 E-02$ & $9.94000 \mathrm{E}-01$ & $3.80000 E-03$ \\
\hline $4.31000 \mathrm{E}-02$ & $9.88800 \mathrm{E}-01$ & $4.00000 \mathrm{E}-03$ & $5.00000 \mathrm{E}-02$ & $9.94900 \mathrm{E}-01$ & $3.70000 \mathrm{E}-03$ \\
\hline $4.31000 \mathrm{E}-02$ & $9.83900 \mathrm{E}-01$ & $3.90000 \mathrm{E}-03$ & $5.00000 E-02$ & $9.93900 \mathrm{E}-01$ & $3.70000 \mathrm{E}-03$ \\
\hline $4.31000 \mathrm{E}-02$ & $9.93600 \mathrm{E}-01$ & $2.70000 \mathrm{E}-03$ & $5.00000 E-02$ & $9.95300 \mathrm{E}-01$ & $3.70000 \mathrm{E}-03$ \\
\hline $4.31000 \mathrm{E}-02$ & $9.95400 \mathrm{E}-01$ & $2.70000 \mathrm{E}-03$ & $5.00000 E-02$ & $9.94800 \mathrm{E}-01$ & $3.70000 \mathrm{E}-03$ \\
\hline $4.31000 \mathrm{E}-02$ & $9.95700 \mathrm{E}-01$ & $2.70000 \mathrm{E}-03$ & $5.00000 \mathrm{E}-02$ & $9.95800 \mathrm{E}-01$ & $3.60000 \mathrm{E}-03$ \\
\hline $4.31000 \mathrm{E}-02$ & $9.95000 \mathrm{E}-01$ & $2.90000 E-03$ & $5.00000 E-02$ & $9.95800 \mathrm{E}-01$ & $3.10000 \mathrm{E}-03$ \\
\hline $4.31000 E-02$ & $9.96600 \mathrm{E}-01$ & $2.80000 \mathrm{E}-03$ & $4.74000 \mathrm{E}-02$ & $9.90600 \mathrm{E}-01$ & $2.40000 E-03$ \\
\hline $4.31000 E-02$ & $9.93900 \mathrm{E}-01$ & $2.80000 \mathrm{E}-03$ & $4.74000 \mathrm{E}-02$ & $9.93000 \mathrm{E}-01$ & $3.60000 \mathrm{E}-03$ \\
\hline $4.31000 \mathrm{E}-02$ & $9.96400 \mathrm{E}-01$ & $2.70000 \mathrm{E}-03$ & $4.74000 E-02$ & $9.99000 E-01$ & $3.40000 \mathrm{E}-03$ \\
\hline $4.31000 \mathrm{E}-02$ & $9.93000 \mathrm{E}-01$ & $2.80000 \mathrm{E}-03$ & $4.74000 \mathrm{E}-02$ & $9.95000 \mathrm{E}-01$ & $2.40000 \mathrm{E}-03$ \\
\hline $4.31000 \mathrm{E}-02$ & $9.94600 \mathrm{E}-01$ & $2.80000 E-03$ & $4.74000 \mathrm{E}-02$ & $9.91500 \mathrm{E}-01$ & $3.60000 \mathrm{E}-03$ \\
\hline $4.31000 \mathrm{E}-02$ & $1.00060 \mathrm{E}+00$ & $2.90000 E-03$ & $4.74000 \mathrm{E}-02$ & $9.88700 \mathrm{E}-01$ & $3.40000 \mathrm{E}-03$ \\
\hline $4.31000 \mathrm{E}-02$ & $9.96700 \mathrm{E}-01$ & $2.70000 \mathrm{E}-03$ & $1.00000 \mathrm{E}-01$ & $9.95200 \mathrm{E}-01$ & $3.90000 \mathrm{E}-03$ \\
\hline $4.31000 \mathrm{E}-02$ & $9.96600 \mathrm{E}-01$ & $2.70000 E-03$ & $1.00000 \mathrm{E}-01$ & $9.94500 \mathrm{E}-01$ & $4.20000 \mathrm{E}-03$ \\
\hline $4.31000 E-02$ & $1.00060 \mathrm{E}+00$ & $2.70000 E-03$ & $1.00000 \mathrm{E}-01$ & $9.98500 \mathrm{E}-01$ & $4.20000 E-03$ \\
\hline $4.31000 \mathrm{E}-02$ & $9.95600 \mathrm{E}-01$ & $2.90000 \mathrm{E}-03$ & $1.00000 \mathrm{E}-01$ & $9.92600 \mathrm{E}-01$ & $4.20000 E-03$ \\
\hline $4.31000 \mathrm{E}-02$ & $9.92300 \mathrm{E}-0 \mathrm{I}$ & $2.70000 \mathrm{E}-03$ & $1.00000 \mathrm{E}-01$ & $9.96000 \mathrm{E}-01$ & $4.80000 \mathrm{E}-03$ \\
\hline $4.31000 \mathrm{E}-02$ & $9.93200 \mathrm{E}-01$ & $2.80000 \mathrm{E}-03$ & $1.00000 \mathrm{E}-01$ & $9.97100 \mathrm{E}-01$ & $4.90000 \mathrm{E}-03$ \\
\hline $4.31000 E-02$ & $1.00180 \mathrm{E}+00$ & $2.90000 \mathrm{E}-03$ & $1.00000 \mathrm{E}-01$ & $9.95200 \mathrm{E}-01$ & $4.90000 \mathrm{E}-03$ \\
\hline $4.31000 \mathrm{E}-02$ & $9.95800 \mathrm{E}-01$ & $2.70000 \mathrm{E}-03$ & $1.00000 \mathrm{E}-01$ & $9.99100 \mathrm{E}-01$ & $5.20000 \mathrm{E}-03$ \\
\hline $4.31000 \mathrm{E}-02$ & $9.93100 \mathrm{E}-01$ & $2.80000 E-03$ & $1.00000 E-01$ & $9.97100 \mathrm{E}-01$ & $5.20000 E-03$ \\
\hline
\end{tabular}


CHPRC-00455, REV. 0

\begin{tabular}{|c|c|c|}
\hline $4.31000 E-02$ & $9.96800 \mathrm{E}-01$ & $2.80000 \mathrm{E}-03$ \\
\hline $4.31000 \mathrm{E}-02$ & $9.95400 \mathrm{E}-01$ & $2.80000 \mathrm{E}-03$ \\
\hline $4.31000 \mathrm{E}-02$ & $9.99400 \mathrm{E}-01$ & $2.70000 \mathrm{E}-03$ \\
\hline $4.31000 E-02$ & $9.95800 \mathrm{E}-01$ & $2.80000 E-03$ \\
\hline $4.31000 \mathrm{E}-02$ & $9.94500 E-01$ & $2.80000 E-03$ \\
\hline $4.31000 \mathrm{E}-02$ & $9.98200 \mathrm{E}-01$ & $2.70000 \mathrm{E}-03$ \\
\hline $4.31000 \mathrm{E}-02$ & $9.89400 \mathrm{E}-01$ & $2.70000 E-03$ \\
\hline $4.31000 E-02$ & $9.92500 \mathrm{E}-01$ & $2.80000 E-03$ \\
\hline $4.31000 E-02$ & $1.00590 \mathrm{E}+00$ & $2.80000 \mathrm{E}-03$ \\
\hline $4.31000 E-02$ & $1.00750 \mathrm{E}+00$ & $2.70000 \mathrm{E}-03$ \\
\hline $4.31000 \mathrm{E}-02$ & $1.00170 \mathrm{E}+00$ & $2.90000 \mathrm{E}-03$ \\
\hline $4.31000 E-02$ & $9.91800 \mathrm{E}-01$ & $2.70000 \mathrm{E}-03$ \\
\hline $4.31000 \mathrm{E}-02$ & $9.94500 \mathrm{E}-01$ & $2.90000 \mathrm{E}-03$ \\
\hline $4.31000 E-02$ & $9.96600 \mathrm{E}-01$ & $2.80000 \mathrm{E}-03$ \\
\hline $4.31000 \mathrm{E}-02$ & $9.96300 \mathrm{E}-01$ & $2.70000 E-03$ \\
\hline $4.31000 \mathrm{E}-02$ & $9.94600 \mathrm{E}-01$ & $2.90000 \mathrm{E}-03$ \\
\hline $4.31000 \mathrm{E}-02$ & $9.98800 \mathrm{E}-01$ & $2.70000 \mathrm{E}-03$ \\
\hline $4.31000 E-02$ & $9.98700 \mathrm{E}-01$ & $2.60000 \mathrm{E}-03$ \\
\hline $4.31000 E-02$ & $9.99300 \mathrm{E}-01$ & $2.80000 \mathrm{E}-03$ \\
\hline $4.31000 E-02$ & $9.97800 \mathrm{E}-01$ & $2.80000 \mathrm{E}-03$ \\
\hline $4.31000 \mathrm{E}-02$ & $9.95000 \mathrm{E}-01$ & $2.80000 E-03$ \\
\hline $4.31000 \mathrm{E}-02$ & $9.96600 \mathrm{E}-01$ & $3.40000 E-03$ \\
\hline $4.31000 \mathrm{E}-02$ & $9.98900 \mathrm{E}-01$ & $3.30000 \mathrm{E}-03$ \\
\hline $4.31000 \mathrm{E}-02$ & $1.00290 \mathrm{E}+00$ & $3.30000 E-03$ \\
\hline $4.31000 \mathrm{E}-02$ & $9.96000 \mathrm{E}-01$ & $3.30000 \mathrm{E}-03$ \\
\hline $4.31000 E-02$ & $9.94200 \mathrm{E}-01$ & $3.40000 \mathrm{E}-03$ \\
\hline $4.31000 \mathrm{E}-02$ & $9.96300 \mathrm{E}-01$ & $3.50000 \mathrm{E}-03$ \\
\hline $4.31000 E-02$ & $1.00330 \mathrm{E}+00$ & $3.40000 \mathrm{E}-03$ \\
\hline $4.31000 \mathrm{E}-02$ & $1.00560 \mathrm{E}+00$ & $3.30000 E-03$ \\
\hline $4.31000 E-02$ & $1.00640 \mathrm{E}+00$ & $3.30000 \mathrm{E}-03$ \\
\hline $4.31000 \mathrm{E}-02$ & $9.97800 \mathrm{E}-01$ & $3.40000 \mathrm{E}-03$ \\
\hline $4.31000 \mathrm{E}-02$ & $9.97800 \mathrm{E}-01$ & $3.30000 E-03$ \\
\hline $4.31000 E-02$ & $9.97800 \mathrm{E}-01$ & $3.30000 \mathrm{E}-03$ \\
\hline $4.31000 \mathrm{E}-02$ & $1.00000 \mathrm{E}+00$ & $3.30000 \mathrm{E}-03$ \\
\hline
\end{tabular}

1. $00000 \mathrm{E}-01$

$1.00000 \mathrm{E}-01$

1. $00000 \mathrm{E}-01$

1. $00000 \mathrm{E}-01$

1. $00000 \mathrm{E}-01$

1. $00000 \mathrm{E}-01$

5. $64000 \mathrm{E}-02$

5. $64000 \mathrm{E}-02$

$5.64000 \mathrm{E}-02$

1. $00000 \mathrm{E}-01$

1. $00000 \mathrm{E}-01$

1. $00000 \mathrm{E}-01$

1. $00000 \mathrm{E}-01$

1. $00000 \mathrm{E}-01$

1. $00000 \mathrm{E}-01$

1. $00000 \mathrm{E}-01$

1. $00000 \mathrm{E}-01$

1. $00000 \mathrm{E}-01$

1. $00000 \mathrm{E}-01$

1. $00000 \mathrm{E}-01$

1. $00000 \mathrm{E}-01$

$1.00000 \mathrm{E}-01$

$1.00000 \mathrm{E}-01$

1. $00000 \mathrm{E}-01$

$1.00000 \mathrm{E}-01$

1. 00000 E-01

1. $00000 \mathrm{E}-01$

1. $00000 \mathrm{E}-01$

1. $00000 \mathrm{E}-01$

1. $00000 \mathrm{E}-01$

$1.00000 \mathrm{E}-01$

1. 00000 E-01
9. $99000 \mathrm{E}-01$

9. $98400 \mathrm{E}-01$

9. $97400 \mathrm{E}-01$

$9.99900 \mathrm{E}-01$

1. $00020 \mathrm{E}+00$

9.99900E-01

9.99500E-01

9.95800E-01

9. $96500 \mathrm{E}-01$

9.97200E-01

9. $98600 \mathrm{E}-01$

1. $00380 \mathrm{E}+00$

9. $96500 \mathrm{E}-01$

9. $98700 \mathrm{E}-01$

9. $99500 \mathrm{E}-01$

9. $96600 \mathrm{E}-01$

9. $99400 \mathrm{E}-01$

9. $96800 \mathrm{E}-01$

9. $98800 \mathrm{E}-0 \mathrm{I}$

9.97400E-01

1. $00380 \mathrm{E}+00$

1. $00490 \mathrm{E}+00$

1. $00160 \mathrm{E}+00$

$1.00240 \mathrm{E}+00$

1. $00010 \mathrm{E}+00$

1. $00220 \mathrm{E}+00$

1. $00170 \mathrm{E}+00$

1. $00350 \mathrm{E}+00$

1. $00270 \mathrm{E}+00$

1. $00030 \mathrm{E}+00$

9. $99800 \mathrm{E}-01$

1. $00180 \mathrm{E}+00$

1. $00050 \mathrm{E}+00$
1. $00000 \mathrm{E}-03$ 1. $10000 \mathrm{E}-03$ 1. $10000 \mathrm{E}-03$ 1. $20000 \mathrm{E}-03$

$1.10000 \mathrm{E}-03$

1. $20000 \mathrm{E}-03$

1. $20000 \mathrm{E}-03$

4. $20000 \mathrm{E}-03$

$5.10000 \mathrm{E}-03$

$6.40000 \mathrm{E}-03$

3. $80000 E-03$

3. $90000 \mathrm{E}-03$

4. $20000 \mathrm{E}-03$

4. $20000 \mathrm{E}-03$

4. $80000 \mathrm{E}-03$

1. $30000 \mathrm{E}-03$

1. $20000 \mathrm{E}-03$

1. $30000 \mathrm{E}-03$

1. 30000 E-03

1. $30000 \mathrm{E}-03$

1. $60000 \mathrm{E}-03$

1. $60000 \mathrm{E}-03$

$1.60000 \mathrm{E}-03$

1. $60000 \mathrm{E}-03$

1. $60000 \mathrm{E}-03$

1. $70000 \mathrm{E}-03$

1. $70000 \mathrm{E}-03$

1. $60000 \mathrm{E}-03$

1. $60000 \mathrm{E}-03$

1. $70000 \mathrm{E}-03$

1. $60000 \mathrm{E}-03$

$1.70000 \mathrm{E}-03$

1. $70000 \mathrm{E}-03$

$\mathrm{chi}=17.8802$ (upper bound $=9.49$ ). The data tests NOT normal

output from statistical treatment

MEU Benchmarks, U-235/U

Number of data points ( $n$ )

Linear regression, $k(x)$

Confidence on fit ( 1 -gamma) [input]

Confidence on proportion (alpha) [input]

Proportion of population falling above

lower tolerance interval (rho) [input]

Minimum value of $x$

Maximum value of $x$

Average value of $x$

Average value of $k$

Minimum value of $\mathrm{k}$

Variance of fit, $s(k, x)^{\wedge} 2$

Within variance, $s(w) \wedge 2$

pooled variance, $s(p)^{\wedge} 2$

Pooled std. deviation, $s(p)$

$C$ (alpha, rho)*s (p)

student-t @(n-2,1-gamma)

Confidence band width, $w$

Minimum margin of subcriticality, $C^{*} s(p)-W$
167

$0.9943+(2.7358 E-02) * X$

$95.0 \%$

$95.0 \%$

$99.5 \%$

$2.3500 \mathrm{E}-02$

$1.7000 \mathrm{E}-01$

$6.1482 \mathrm{E}-02$

0.99596

0.97460

$2.6283 E-05$

1. $1262 \mathrm{E}-05$

$3.7545 \mathrm{E}-05$

$6.1274 \mathrm{E}-03$

$2.6851 E-02$

1. $64500 \mathrm{E}+00$

$1.0450 \mathrm{E}-02$

$1.6400 \mathrm{E}-02$

Upper subcritical limits: $(2.35000 \mathrm{E}-02<=\mathrm{X}<=0.17000)$

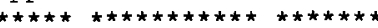

USL Method I (Confidence Band with

Administrative Margin)

USL1 $=0.9338+(2.7358 E-02) * X$

USI Method 2 (Single-Sided Uniform

width Closed Interval Approach) USL2 $=0.9674+(2.7358 \mathrm{E}-02) \star \mathrm{X}$

USLs Evaluated Over Range of Parameter X:

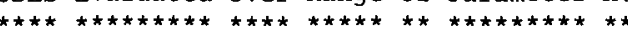




\section{CHPRC-00455, REV. 0}

\begin{tabular}{|c|c|c|c|c|c|c|c|c|}
\hline $\mathrm{X}:$ & $2.35 \mathrm{E}-2$ & $4.44 \mathrm{E}-2$ & $6.54 \mathrm{E}-2$ & $8.63 E-2$ & $1.07 \mathrm{E}-1$ & $1.28 \mathrm{E}-1$ & $1.49 \mathrm{E}-1$ & $1.70 \mathrm{E}-1$ \\
\hline USL- 1 : & 0.9345 & 0.9350 & 0.9356 & 0.9362 & 0.9368 & 0.9373 & 0.9379 & 0.9385 \\
\hline USL-2: & 0.9681 & 0.9686 & 0.9692 & 0.9698 & 0.9704 & 0.9709 & 0.9715 & 0.9721 \\
\hline & & & & & & & & \\
\hline & & & 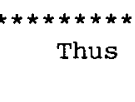 & $\begin{array}{l}\text { spake USLS } \\
\text { Finis. }\end{array}$ & $\begin{array}{l}\star \star \star \star \star \star \star \star \star \star \star \star ~ \\
\text { STATS }\end{array}$ & & & \\
\hline
\end{tabular}

D-10 
uslstats: a utility to calculate upper subcritical

limits for criticality safety applications

Version 1.4, April 23, 2003

Oak Ridge National Laboratory

Input to statistical treatment from file:meu-Vm_vf.inp

Title: MEU Benchmarks, Vm/Vf

$\begin{array}{llr}\text { Proportion of the population } & =.995 \\ \text { Confidence of } \mathrm{fit} & =.950 \\ \text { Confidence on proportion } & =.950 \\ \text { Number of observations } & =167 \\ \text { Minimum value of closed band } & =0.00 \\ \text { Maximum value of closed band } & =0.00 \\ \text { Administrative margin } & =0.05\end{array}$

\begin{tabular}{|c|c|c|c|c|c|}
\hline independent & dependent & deviation & independent & dependent & deviation \\
\hline variable - $x$ & variable - y & in $y$ & variable $-x$ & variable - y & in $y$ \\
\hline $7.15000 \mathrm{E}+00$ & $9.92200 \mathrm{E}-01$ & $4.00000 \mathrm{E}-03$ & $1.59700 \mathrm{E}+00$ & $1.00150 \mathrm{E}+00$ & $3.30000 E-03$ \\
\hline 7. $15000 \mathrm{E}+00$ & $9.95600 \mathrm{E}-01$ & $4.10000 E-03$ & 1. $59700 \mathrm{E}+00$ & $9.96100 \mathrm{E}-01$ & $3.40000 \mathrm{E}-03$ \\
\hline $7.15000 \mathrm{E}+00$ & $9.96900 \mathrm{E}-01$ & $4.50000 \mathrm{E}-03$ & $1.59700 \mathrm{E}+00$ & $9.99000 \mathrm{E}-01$ & $3.40000 E-03$ \\
\hline $7.15000 \mathrm{E}+00$ & $9.91400 \mathrm{E}-01$ & $4.50000 E-03$ & $1.59700 \mathrm{E}+00$ & $9.92100 \mathrm{E}-01$ & $3.30000 E-03$ \\
\hline $7.15000 E+00$ & $9.91700 \mathrm{E}-01$ & $4.40000 E-03$ & $2.14930 \mathrm{E}+00$ & $9.99000 \mathrm{E}-01$ & $3.50000 \mathrm{E}-03$ \\
\hline $7.15000 \mathrm{E}+00$ & $9.85900 \mathrm{E}-01$ & $4.50000 E-03$ & $2.14930 \mathrm{E}+00$ & $1.00510 \mathrm{E}+00$ & $3.50000 \mathrm{E}-03$ \\
\hline $3.88210 \mathrm{E}+00$ & $9.93200 E-01$ & $2.80000 E-03$ & $1.22370 E+00$ & $9.98700 E-01$ & $6.20000 \mathrm{E}-03$ \\
\hline $3.88210 \mathrm{E}+00$ & $9.95000 \mathrm{E}-01$ & $2.80000 \mathrm{E}-03$ & $1.22370 \mathrm{E}+00$ & $1.00770 \mathrm{E}+00$ & $6.20000 \mathrm{E}-03$ \\
\hline $3.88210 \mathrm{E}+00$ & $9.99700 \mathrm{E}-01$ & $2.80000 E-03$ & $8.97000 \mathrm{E}-01$ & $9.98400 \mathrm{E}-01$ & $4.20000 \mathrm{E}-03$ \\
\hline $3.88210 \mathrm{E}+00$ & $9.98600 \mathrm{E}-01$ & $2.80000 \mathrm{E}-03$ & $8.97000 E-01$ & $1.00650 \mathrm{E}+00$ & $4.20000 \mathrm{E}-03$ \\
\hline $3.88210 \mathrm{E}+00$ & $9.94500 E-01$ & $2.80000 \mathrm{E}-03$ & $1.59950 \mathrm{E}+00$ & $9.96900 \mathrm{E}-01$ & $2.30000 \mathrm{E}-03$ \\
\hline $1.59700 E+00$ & $9.92500 \mathrm{E}-01$ & $3.80000 \mathrm{E}-03$ & $1.59950 \mathrm{E}+00$ & $9.93200 \mathrm{E}-01$ & $2.30000 \mathrm{E}-03$ \\
\hline $1.59700 \mathrm{E}+00$ & $9.89200 \mathrm{E}-01$ & $4.00000 \mathrm{E}-03$ & $1.59950 \mathrm{E}+00$ & $9.96800 \mathrm{E}-01$ & $2.20000 \mathrm{E}-03$ \\
\hline $1.59700 \mathrm{E}+00$ & $9.93700 \mathrm{E}-01$ & $3.90000 \mathrm{E}-03$ & 1. $59950 \mathrm{E}+00$ & $9.92700 \mathrm{E}-01$ & $2.40000 E-03$ \\
\hline $1.59700 \mathrm{E}+00$ & $9.90000 \mathrm{E}-01$ & $3.80000 E-03$ & $1.59950 \mathrm{E}+00$ & $9.95700 \mathrm{E}-01$ & $3.70000 \mathrm{E}-03$ \\
\hline $1.59700 \mathrm{E}+00$ & $9.92300 \mathrm{E}-01$ & $3.80000 \mathrm{E}-03$ & $1.59950 \mathrm{E}+00$ & $9.96600 \mathrm{E}-01$ & $2.30000 E-03$ \\
\hline $1.59700 \mathrm{E}+00$ & $9.87200 \mathrm{E}-01$ & $4.00000 \mathrm{E}-03$ & $1.59950 \mathrm{E}+00$ & $9.94200 \mathrm{E}-01$ & $2.40000 \mathrm{E}-03$ \\
\hline $1.59700 \mathrm{E}+00$ & $9.89300 E-01$ & $3.90000 \mathrm{E}-03$ & $2.01240 E+00$ & $9.95000 \mathrm{E}-01$ & $3.50000 \mathrm{E}-03$ \\
\hline $1.59700 \mathrm{E}+00$ & $9.85300 \mathrm{E}-01$ & $4.10000 \mathrm{E}-03$ & $2.01240 \mathrm{E}+00$ & $9.94700 \mathrm{E}-01$ & $3.40000 \mathrm{E}-03$ \\
\hline $1.59700 \mathrm{E}+00$ & $9.85500 \mathrm{E}-01$ & $4.00000 E-03$ & $2.01240 E+00$ & $9.94700 \mathrm{E}-01$ & $3.50000 \mathrm{E}-03$ \\
\hline $1.59700 \mathrm{E}+00$ & $9.97100 \mathrm{E}-01$ & $4.00000 E-03$ & $2.01240 E+00$ & $9.94800 \mathrm{E}-01$ & $3.50000 \mathrm{E}-03$ \\
\hline $1.59700 \mathrm{E}+00$ & $9.83000 \mathrm{E}-01$ & $4.00000 \mathrm{E}-03$ & $2.51640 \mathrm{E}+00$ & $9.95700 \mathrm{E}-01$ & $4.20000 \mathrm{E}-03$ \\
\hline $1.59700 \mathrm{E}+00$ & $9.74600 \mathrm{E}-01$ & $3.90000 E-03$ & $2.51640 \mathrm{E}+00$ & $9.96100 \mathrm{E}-01$ & $4.30000 \mathrm{E}-03$ \\
\hline $1.59700 \mathrm{E}+00$ & $9.82000 \mathrm{E}-01$ & $4.00000 E-03$ & $2.51640 \mathrm{E}+00$ & $9.95300 \mathrm{E}-01$ & $4.20000 \mathrm{E}-03$ \\
\hline $1.59700 \mathrm{E}+00$ & $9.88100 \mathrm{E}-01$ & $4.20000 \mathrm{E}-03$ & $2.51640 \mathrm{E}+00$ & $9.93000 \mathrm{E}-01$ & $4.20000 E-03$ \\
\hline $1.59700 \mathrm{E}+00$ & $9.82500 \mathrm{E}-01$ & $4.00000 \mathrm{E}-03$ & $3.00670 \mathrm{E}+00$ & $9.94800 E-01$ & $3.80000 E-03$ \\
\hline $1.59700 \mathrm{E}+00$ & $9.86900 E-01$ & $4.00000 \mathrm{E}-03$ & $3.00670 E+00$ & $9.97000 \mathrm{E}-01$ & $3.80000 \mathrm{E}-03$ \\
\hline $1.59700 \mathrm{E}+00$ & $9.85600 \mathrm{E}-01$ & $4.00000 E-03$ & $3.00670 E+00$ & $9.92800 \mathrm{E}-01$ & $3.80000 \mathrm{E}-03$ \\
\hline $1.59700 \mathrm{E}+00$ & $9.82000 \mathrm{E}-01$ & $4.00000 E-03$ & $3.00670 E+00$ & $9.94000 E-01$ & $3.80000 \mathrm{E}-03$ \\
\hline $1.59700 \mathrm{E}+00$ & $9.88800 \mathrm{E}-01$ & $4.00000 \mathrm{E}-03$ & $3.00670 \mathrm{E}+00$ & $9.94900 \mathrm{E}-01$ & $3.70000 \mathrm{E}-03$ \\
\hline $1.59700 \mathrm{E}+00$ & $9.83900 \mathrm{E}-01$ & $3.90000 E-03$ & $3.00670 \mathrm{E}+00$ & $9.93900 E-01$ & $3.70000 \mathrm{E}-03$ \\
\hline $3.88210 \mathrm{E}+00$ & $9.93600 \mathrm{E}-01$ & $2.70000 \mathrm{E}-03$ & $3.00670 \mathrm{E}+00$ & $9.95300 \mathrm{E}-01$ & $3.70000 \mathrm{E}-03$ \\
\hline $3.88210 \mathrm{E}+00$ & $9.95400 \mathrm{E}-01$ & $2.70000 \mathrm{E}-03$ & $3.00670 \mathrm{E}+00$ & $9.94800 \mathrm{E}-01$ & $3.70000 \mathrm{E}-03$ \\
\hline $3.88210 E+00$ & $9.95700 \mathrm{E}-01$ & $2.70000 \mathrm{E}-03$ & $3.00670 \mathrm{E}+00$ & $9.95800 \mathrm{E}-01$ & $3.60000 \mathrm{E}-03$ \\
\hline $3.88210 \mathrm{E}+00$ & $9.95000 \mathrm{E}-01$ & $2.90000 E-03$ & $3.00670 \mathrm{E}+00$ & $9.95800 \mathrm{E}-01$ & $3.10000 \mathrm{E}-03$ \\
\hline $3.88210 \mathrm{E}+00$ & $9.96600 \mathrm{E}-01$ & $2.80000 \mathrm{E}-03$ & $2.14260 \mathrm{E}+00$ & $9.90600 \mathrm{E}-01$ & $2.40000 \mathrm{E}-03$ \\
\hline $3.88210 \mathrm{E}+00$ & $9.93900 E-01$ & $2.80000 \mathrm{E}-03$ & $4.14950 E+00$ & $9.93000 \mathrm{E}-01$ & $3.60000 \mathrm{E}-03$ \\
\hline $3.88210 \mathrm{E}+00$ & $9.96400 E-01$ & $2.70000 E-03$ & $7.94660 \mathrm{E}+00$ & $9.99000 \mathrm{E}-01$ & $3.40000 \mathrm{E}-03$ \\
\hline $3.88210 \mathrm{E}+00$ & $9.93000 \mathrm{E}-01$ & $2.80000 \mathrm{E}-03$ & $2.14260 \mathrm{E}+00$ & $9.95000 \mathrm{E}-01$ & $2.40000 \mathrm{E}-03$ \\
\hline $3.88210 \mathrm{E}+00$ & $9.94600 \mathrm{E}-01$ & $2.80000 \mathrm{E}-03$ & $4.14950 E+00$ & $9.91500 \mathrm{E}-01$ & $3.60000 \mathrm{E}-03$ \\
\hline $3.88210 \mathrm{E}+00$ & $1.00060 \mathrm{E}+00$ & $2.90000 \mathrm{E}-03$ & $7.94660 \mathrm{E}+00$ & $9.88700 \mathrm{E}-01$ & $3.40000 \mathrm{E}-03$ \\
\hline $3.88210 \mathrm{E}+00$ & $9.96700 \mathrm{E}-01$ & $2.70000 \mathrm{E}-03$ & $7.84942 \mathrm{E}+01$ & $9.95200 \mathrm{E}-01$ & $3.90000 \mathrm{E}-03$ \\
\hline $3.88210 \mathrm{E}+00$ & $9.96600 \mathrm{E}-01$ & $2.70000 \mathrm{E}-03$ & $8.94269 \mathrm{E}+01$ & $9.94500 \mathrm{E}-01$ & $4.20000 \mathrm{E}-03$ \\
\hline $3.88210 \mathrm{E}+00$ & 1. $00060 \mathrm{E}+00$ & $2.70000 \mathrm{E}-03$ & $9.14054 \mathrm{E}+01$ & $9.98500 \mathrm{E}-01$ & $4.20000 E-03$ \\
\hline $3.88210 \mathrm{E}+00$ & $9.95600 \mathrm{E}-01$ & $2.90000 \mathrm{E}-03$ & $9.30594 \mathrm{E}+01$ & $9.92600 \mathrm{E}-01$ & $4.20000 \mathrm{E}-03$ \\
\hline $3.88210 \mathrm{E}+00$ & $9.92300 \mathrm{E}-01$ & $2.70000 E-03$ & $1.19568 \mathrm{E}+02$ & $9.96000 \mathrm{E}-01$ & $4.80000 E-03$ \\
\hline $3.88210 \mathrm{E}+00$ & $9.93200 \mathrm{E}-01$ & $2.80000 \mathrm{E}-03$ & 1. $23615 \mathrm{E}+02$ & $9.97100 \mathrm{E}-01$ & $4.90000 E-03$ \\
\hline $3.88210 \mathrm{E}+00$ & $1.00180 \mathrm{E}+00$ & $2.90000 \mathrm{E}-03$ & 1. $26336 \mathrm{E}+02$ & $9.95200 \mathrm{E}-01$ & $4.90000 E-03$ \\
\hline $3.88210 \mathrm{E}+00$ & $9.95800 \mathrm{E}-01$ & $2.70000 E-03$ & $1.43847 \mathrm{E}+02$ & $9.99100 \mathrm{E}-01$ & $5.20000 E-03$ \\
\hline $3.88210 \mathrm{E}+00$ & $9.93100 \mathrm{E}-01$ & $2.80000 \mathrm{E}-03$ & 1. $46481 \mathrm{E}+02$ & $9.97100 \mathrm{E}-01$ & $5.20000 E-03$ \\
\hline
\end{tabular}




\section{CHPRC-00455, REV. 0}

\begin{tabular}{|c|c|c|c|c|c|}
\hline $3.88210 E+00$ & $9.96800 \mathrm{E}-01$ & $2.80000 E-03$ & $0.00000 E+00$ & $9.99000 \mathrm{E}-01$ & $1.00000 \mathrm{E}-03$ \\
\hline $3.88210 \mathrm{E}+00$ & $9.95400 \mathrm{E}-01$ & $2.80000 \mathrm{E}-03$ & $0.00000 \mathrm{E}+00$ & $9.98400 \mathrm{E}-01$ & $1.10000 \mathrm{E}-03$ \\
\hline $3.88210 \mathrm{E}+00$ & $9.99400 \mathrm{E}-01$ & $2.70000 \mathrm{E}-03$ & $0.00000 \mathrm{E}+00$ & $9.97400 \mathrm{E}-01$ & $1.10000 \mathrm{E}-03$ \\
\hline $3.88210 \mathrm{E}+00$ & $9.95800 E-01$ & $2.80000 \mathrm{E}-03$ & $0.00000 \mathrm{E}+00$ & $9.99900 \mathrm{E}-01$ & $1.20000 \mathrm{E}-03$ \\
\hline $3.88210 \mathrm{E}+00$ & $9.94500 \mathrm{E}-01$ & $2.80000 E-03$ & $0.00000 \mathrm{E}+00$ & I. $00020 \mathrm{E}+00$ & $1.10000 \mathrm{E}-03$ \\
\hline $3.88210 \mathrm{E}+00$ & $9.98200 \mathrm{E}-01$ & $2.70000 \mathrm{E}-03$ & $0.00000 \mathrm{E}+00$ & $9.99900 E-01$ & $1.20000 \mathrm{E}-03$ \\
\hline $3.88210 \mathrm{E}+00$ & $9.89400 \mathrm{E}-0 \mathrm{I}$ & $2.70000 E-03$ & $0.00000 \mathrm{E}+00$ & $9.99500 \mathrm{E}-01$ & $1.20000 \mathrm{E}-03$ \\
\hline $3.88210 \mathrm{E}+00$ & $9.92500 \mathrm{E}-01$ & $2.80000 \mathrm{E}-03$ & $5.54913 E+01$ & $9.95800 \mathrm{E}-01$ & $4.20000 \mathrm{E}-03$ \\
\hline $3.88210 \mathrm{E}+00$ & $1.00590 \mathrm{E}+00$ & $2.80000 E-03$ & $5.54913 \mathrm{E}+01$ & $9.96500 \mathrm{E}-01$ & $5.10000 \mathrm{E}-03$ \\
\hline $3.88210 \mathrm{E}+00$ & $1.00750 \mathrm{E}+00$ & $2.70000 E-03$ & $5.54913 E+01$ & $9.97200 \mathrm{E}-01$ & $6.40000 E-03$ \\
\hline $3.88210 \mathrm{E}+00$ & $1.00170 E+00$ & $2.90000 \mathrm{E}-03$ & $5.41652 \mathrm{E}+01$ & $9.98600 \mathrm{E}-01$ & $3.80000 \mathrm{E}-03$ \\
\hline $3.88210 \mathrm{E}+00$ & $9.91800 \mathrm{E}-01$ & $2.70000 E-03$ & $5.41652 \mathrm{E}+01$ & $1.00380 \mathrm{E}+00$ & $3.90000 \mathrm{E}-03$ \\
\hline $3.88210 \mathrm{E}+00$ & $9.94500 \mathrm{E}-01$ & $2.90000 E-03$ & $5.41652 \mathrm{E}+01$ & $9.96500 \mathrm{E}-01$ & $4.20000 \mathrm{E}-03$ \\
\hline $3.88210 E+00$ & $9.96600 \mathrm{E}-01$ & $2.80000 \mathrm{E}-03$ & $5.41652 \mathrm{E}+01$ & $9.98700 \mathrm{E}-01$ & $4.20000 \mathrm{E}-03$ \\
\hline $3.88210 E+00$ & $9.96300 \mathrm{E}-01$ & $2.70000 \mathrm{E}-03$ & $5.41652 \mathrm{E}+01$ & $9.99500 \mathrm{E}-01$ & $4.80000 \mathrm{E}-03$ \\
\hline $3.88210 \mathrm{E}+00$ & $9.94600 \mathrm{E}-01$ & $2.90000 \mathrm{E}-03$ & $7.15241 \mathrm{E}+0 \mathrm{I}$ & $9.96600 \mathrm{E}-01$ & $1.30000 \mathrm{E}-03$ \\
\hline $3.88210 E+00$ & $9.98800 \mathrm{E}-01$ & $2.70000 \mathrm{E}-03$ & $7.76479 E+01$ & $9.99400 \mathrm{E}-01$ & $1.20000 \mathrm{E}-03$ \\
\hline $3.88210 E+00$ & $9.98700 \mathrm{E}-01$ & $2.60000 \mathrm{E}-03$ & $8.49302 E+01$ & $9.96800 \mathrm{E}-01$ & $1.30000 \mathrm{E}-03$ \\
\hline $3.88210 E+00$ & $9.99300 \mathrm{E}-01$ & $2.80000 E-03$ & $9.03621 E+01$ & $9.98800 E-01$ & $1.30000 \mathrm{E}-03$ \\
\hline $3.88210 \mathrm{E}+00$ & $9.97800 \mathrm{E}-01$ & $2.80000 \mathrm{E}-03$ & $9.50205 E+01$ & $9.97400 \mathrm{E}-01$ & $1.30000 \mathrm{E}-03$ \\
\hline $3.88210 \mathrm{E}+00$ & $9.95000 \mathrm{E}-01$ & $2.80000 \mathrm{E}-03$ & 4. $72695 E+01$ & $1.00380 \mathrm{E}+00$ & $1.60000 \mathrm{E}-03$ \\
\hline $1.59700 \mathrm{E}+00$ & $9.96600 \mathrm{E}-01$ & $3.40000 E-03$ & $5.18479 E+01$ & $1.00490 \mathrm{E}+00$ & $1.60000 \mathrm{E}-03$ \\
\hline $1.59700 \mathrm{E}+00$ & $9.98900 \mathrm{E}-01$ & $3.30000 \mathrm{E}-03$ & $6.13569 \mathrm{E}+01$ & $1.00160 \mathrm{E}+00$ & $1.60000 \mathrm{E}-03$ \\
\hline $1.59700 E+00$ & $1.00290 E+00$ & $3.30000 \mathrm{E}-03$ & $6.55709 \mathrm{E}+01$ & $1.00240 \mathrm{E}+00$ & $1.60000 \mathrm{E}-03$ \\
\hline $1.59700 \mathrm{E}+00$ & $9.96000 E-01$ & $3.30000 E-03$ & $7.05048 \mathrm{E}+01$ & $1.00010 E+00$ & $1.60000 \mathrm{E}-03$ \\
\hline $1.59700 \mathrm{E}+00$ & $9.94200 \mathrm{E}-01$ & $3.40000 \mathrm{E}-03$ & $7.45171 \mathrm{E}+01$ & $1.00220 \mathrm{E}+00$ & $1.70000 \mathrm{E}-03$ \\
\hline $1.59700 \mathrm{E}+00$ & $9.96300 \mathrm{E}-01$ & $3.50000 E-03$ & $7.78317 \mathrm{E}+01$ & $1.00170 \mathrm{E}+00$ & $1.70000 \mathrm{E}-03$ \\
\hline $1.59700 \mathrm{E}+00$ & $1.00330 \mathrm{E}+00$ & $3.40000 E-03$ & $4.72695 \mathrm{E}+01$ & $1.00350 \mathrm{E}+00$ & $1.60000 \mathrm{E}-03$ \\
\hline $1.59700 E+00$ & $1.00560 \mathrm{E}+00$ & $3.30000 \mathrm{E}-03$ & $5.15151 E+01$ & $1.00270 \mathrm{E}+00$ & $1.60000 \mathrm{E}-03$ \\
\hline $1.59700 \mathrm{E}+00$ & 1. $00640 \mathrm{E}+00$ & $3.30000 \mathrm{E}-03$ & $6.16113 E+01$ & $1.00030 \mathrm{E}+00$ & $1.70000 \mathrm{E}-03$ \\
\hline $1.59700 \mathrm{E}+00$ & $9.97800 \mathrm{E}-01$ & $3.40000 \mathrm{E}-03$ & $6.55572 \mathrm{E}+01$ & $9.99800 \mathrm{E}-01$ & $1.60000 \mathrm{E}-03$ \\
\hline $1.59700 \mathrm{E}+00$ & $9.97800 \mathrm{E}-01$ & $3.30000 E-03$ & $7.05120 \mathrm{E}+01$ & 1. $00180 \mathrm{E}+00$ & $1.70000 \mathrm{E}-03$ \\
\hline 1. $59700 \mathrm{E}+00$ & $9.97800 \mathrm{E}-01$ & $3.30000 E-03$ & $7.35156 \mathrm{E}+01$ & $1.00050 \mathrm{E}+00$ & $1.70000 \mathrm{E}-03$ \\
\hline $1.59700 \mathrm{E}+00$ & $1.00000 \mathrm{E}+00$ & $3.30000 E-03$ & & & \\
\hline
\end{tabular}

$\operatorname{chi}=17.8802$ (upper bound $=9.49$ ). The data tests NoT normal

Output from statistical treatment

MEU Benchmarks, $\quad \mathrm{m} / \mathrm{Vf}$

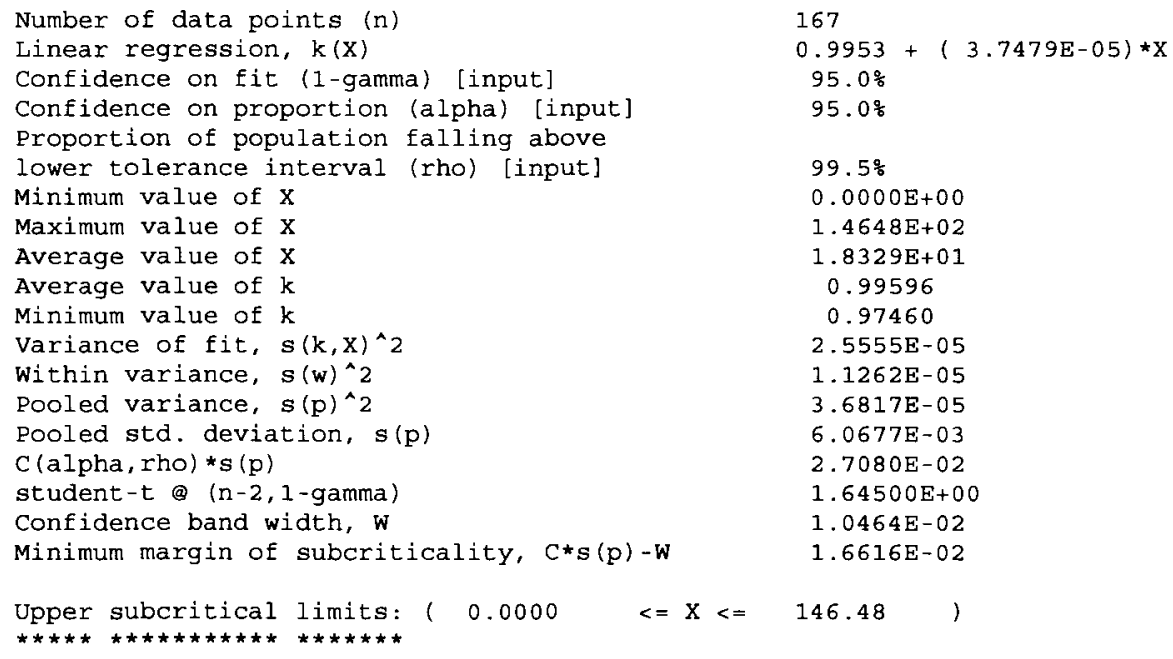

USL Method 1 (Confidence Band with

Administrative Margin) USLl $=0.9348+(3.7479 \mathrm{E}-05) * \mathrm{X}(\mathrm{X}<126.06)$ $=0.9395 \quad(X>=126.06)$

USL Method 2 (Single-Sided Uniform

Width Closed Interval Approach) USL2 $=0.9682+(3.7479 \mathrm{E}-05) \star \mathrm{X}(\mathrm{X}<1.26061 \mathrm{E}+02)$
$=0.9729$
$(X>=1.26061 \mathrm{E}+02)$

USLs Evaluated Over Range of Parameter $\mathrm{X}$ : 


\section{CHPRC-00455, REV. 0}

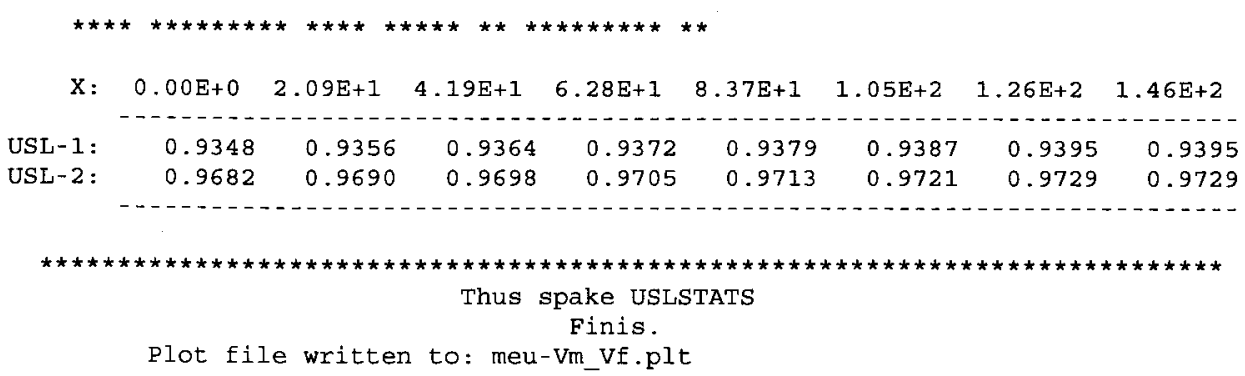

D-13 
CHPRC-00455, REV. 0

This page intentionally left blank

D-14 
CHPRC-00455, REV. 0

APPENDIX E - MANAGEMENT DECISION

E-1 
CHPRC-00455, REV. 0

This page is intentionally left blank 
From: Puigh, Raymond

Sent: Wednesday, August 16, 2006 2:41 PM

To: Miller, Edward M; Lan, Jay S

Cc: Puigh, Raymond; Erickson, David G; Nelson, Joe

Subject: MCNP Version 5

\section{Gentlemen,}

I have decided to proceed with the issuance of MCNP Version 5 using the current validation database used for the version $4 \mathrm{C}$ validation. After documentation has been completed we will address a review of the database for new data that should be added to the validation database and update our documentation. If you have any questions, please call at 376-3766.

RJ Puigh 
CHPRC-00455, REV. 0

This page is intentionally left blank. 